\title{
The Effect of Exhaust Plume/Afterbody Interaction on Installed Scramjet Performance
}

Thomas A. Edwards

(MASA-IA-101033) THE EFEECT CF EXHAUST ELOAE, AFIERECIY INTEEACIICA CA ILSTALLED

SCFABJET FERFCELACE (NASA) EC F CSCL $21 E$
$89-176 C 0$

Unclas

0191240

December 1988

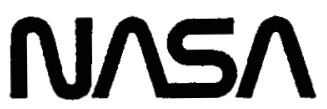

National Aeronautics and Space Administration 
Thomas A. Edwards, Ames Research Center, Moffett Field, Califomia

National Aeronautics and

Space Administration

Ames Research Center

Moffett Field, California 94035 


\title{
THE EFFECT OF EXHAUST PLUME/AFTERBODY INTERACTION ON INSTALLED SCRAMJET PERFORMANCE
}

\author{
Thomas Alan Edwards \\ NASA Ames Research Center, Moffett Field, CA 94035
}

\section{SUMMARY}

Newly emerging aerospace technology points to the feasibility of sustained hypersonic flight. Designing a propulsion system capable of generating the necessary thrust is now the major obstacle. Firstgeneration vehicles will be driven by air-breathing scramjet (supersonic combustion ramjet) engines. Because of engine size limitations, the exhaust gas leaving the nozzle will be highly underexpanded. Consequently, a significant amount of thrust and lift can be extracted by allowing the exhaust gases to expand along the underbody of the vehicle. Predicting how these forces influence overall vehicle thrust, lift, and moment is essential to a successful design. This work represents an important first step toward that objective. The UWIN code, an upwind, implicit Navier-Stokes computer program, has been applied to hypersonic exhaust plume/afterbody flow fields. The capability to solve entire vehicle geometries at hypersonic speeds, including an interacting exhaust plume, has been demonstrated for the first time to the author's knowledge. Comparison of the numerical results with available experimental data shows good agreement in all cases investigated. For moderately underexpanded jets, afterbody forces were found to vary linearly with the nozzle exit pressure, and increasing the exit pressure produced additional nose-down pitching moment. Coupling a species continuity equation to the UWIN code enabled calculations indicating that exhaust gases with low isentropic exponents $(\gamma)$ contribute larger afterbody forces than high- $\gamma$ exhaust gases. Moderately underexpanded jets, which remain attached to unswept afterbodies, underwent streamwise separation on upswept afterbodies. Highly underexpanded jets produced altogether different flow patterns, however. The highly underexpanded jet creates a strong plume shock, and the interaction of this shock with the afterbody was found to produce complicated patterns of crossflow separation. Finally, the effect of thrust vectoring on vehicle balance has been shown to alter dramatically the vehicle pitching moment.

PRECEDÜY PRGE BLARK NOT FILMED

iii 


\section{PREFACE}

Hypersonic flight represents the newest frontier in man's quest to fly higher and faster. Unique among aerospace vehicles, hypersonic craft will roam the diffuse boundary between conventional atmospheric flight and space flight; they will bridge a gap in the increasingly traveled path to space. But to do so will require quantum technology leaps in several aerospace disciplines. Scramjet propulsion, at the fore of hypersonic research activity, provides the impetus for computational aerothermodynamicists to address this problem. Applying their approach to develop a predictive method for hypersonic vehicle flow fields will facilitate the design process at all stages. Progress toward this goal is being pursued along several fronts: geometry definition and grid generation, flow modeling, algorithm development, code calibration, detailed component studies, and configuration studies.

Within the context of scramjet exhaust plume flows, this work attempts to contribute to each of these categories, albeit in varying degrees. By encompassing some specific and general aspects of plume flows, the investigation offers insight into parameters that govern the problem. It also reflects the current status of predictive capabilities for these flows. The following pages describe this investigation in its entirety. Relatively new aspects of the problem to computational methods, such as coupling a species continuity equation or implementation of the total-variation-diminishing (TVD) scheme, are presented in some detail and are referenced for further reading. However, other aspects standard to computational fluid dynamics are addressed only insofar as they impact the present discussion: the Baldwin-Lomax turbulence model, for example. 


\section{TABLE OF CONTENTS}

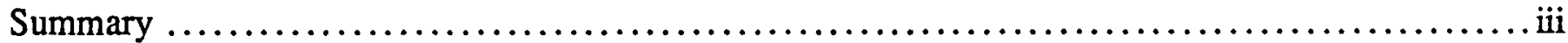

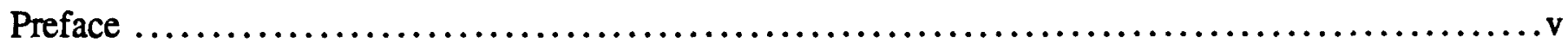

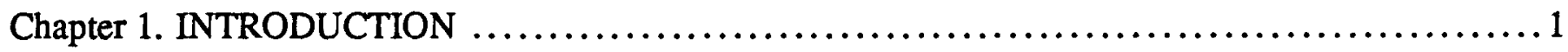

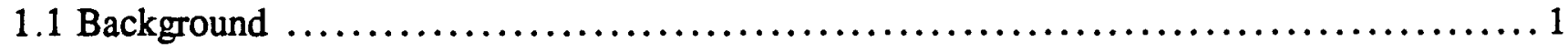

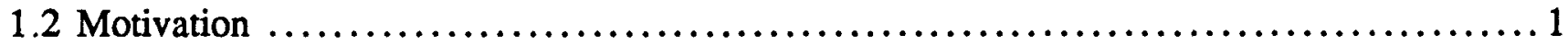

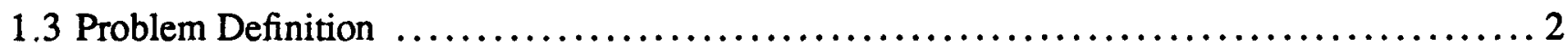

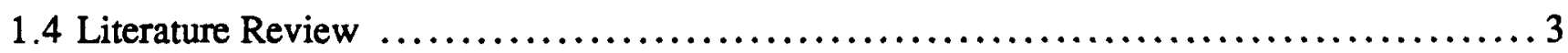

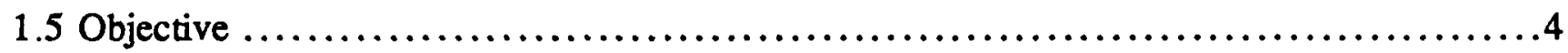

Chapter 2. GOVERNING EQUATIONS AND NUMERICAL METHODS $\ldots \ldots \ldots \ldots \ldots \ldots \ldots \ldots \ldots$

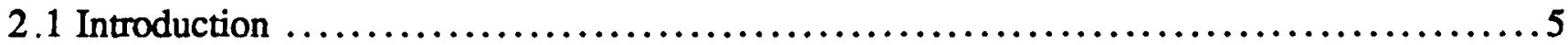

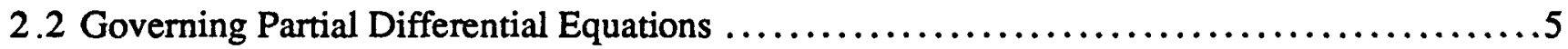

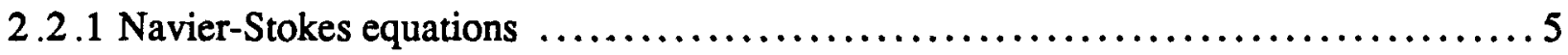

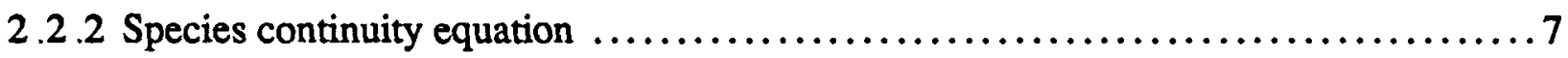

2.2 .3 Coupling the equation sets $\ldots \ldots \ldots \ldots \ldots \ldots \ldots \ldots \ldots \ldots \ldots \ldots \ldots \ldots \ldots \ldots$

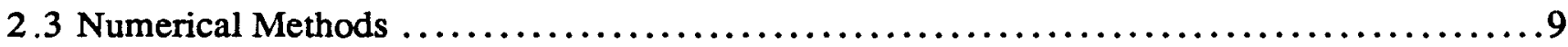

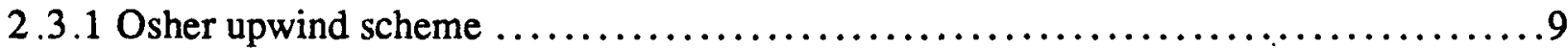

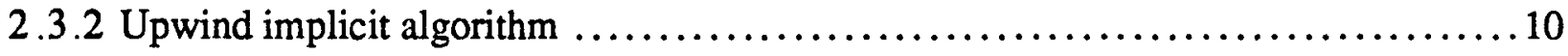

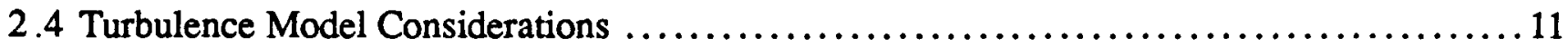

Chapter 3. ANALYSIS OF NOZZLE/AFTERBODY FLOW FIELDS $\ldots \ldots \ldots \ldots \ldots \ldots \ldots \ldots \ldots \ldots$

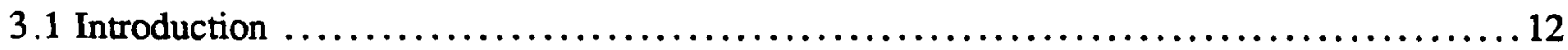

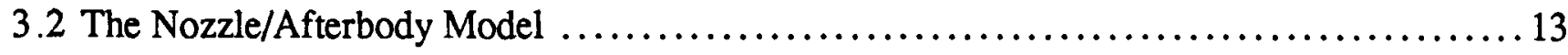

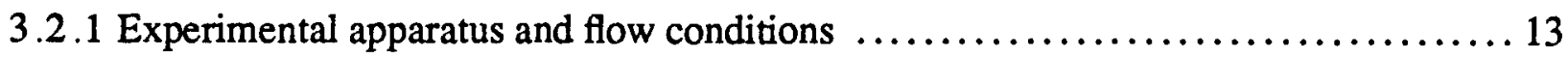




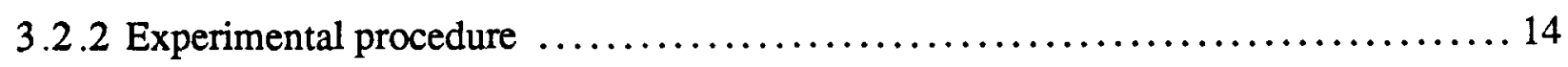

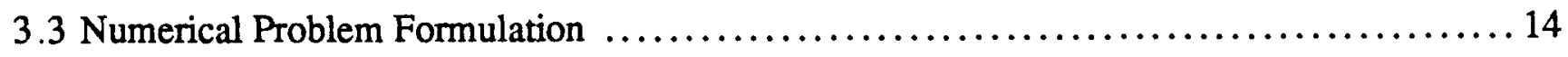

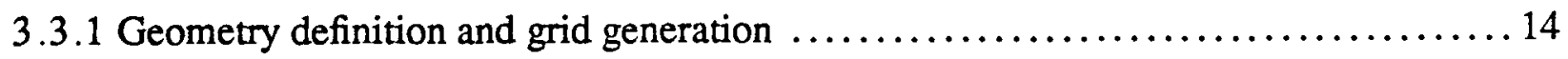

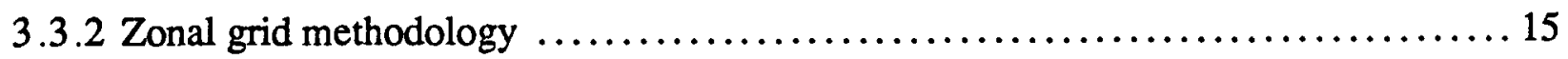

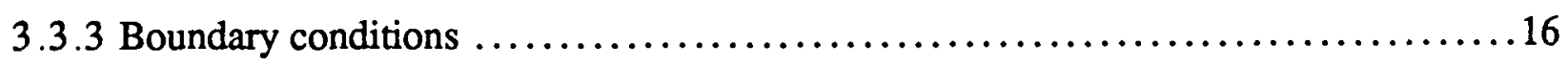

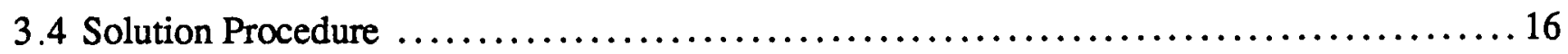

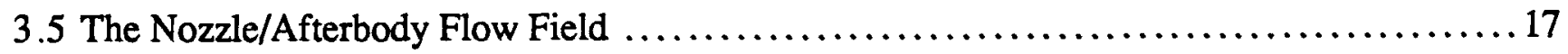

3.6 The Influence of Parametric Variations on Afterbody Forces . ....................... 19

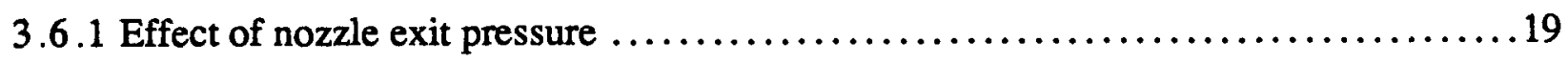

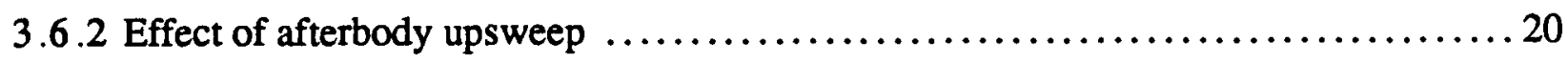

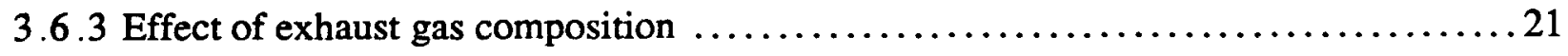

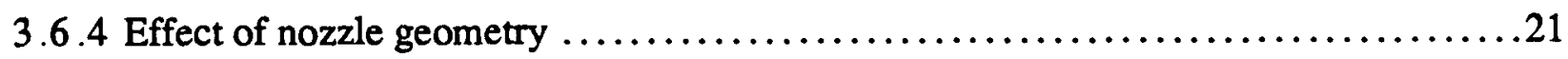

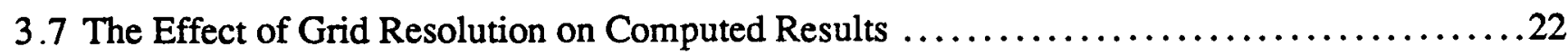

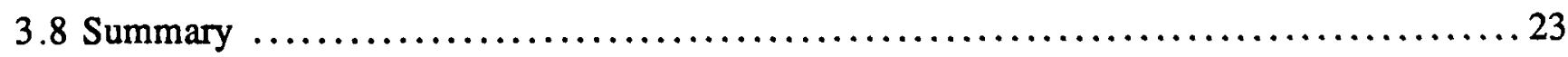

Chapter 4. ANALYSIS OF GENERIC HYPERSONIC VEHICLE

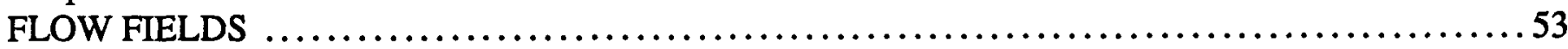

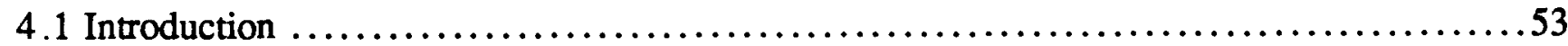

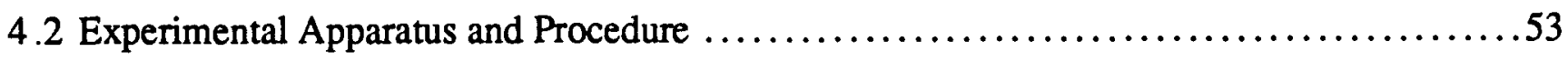

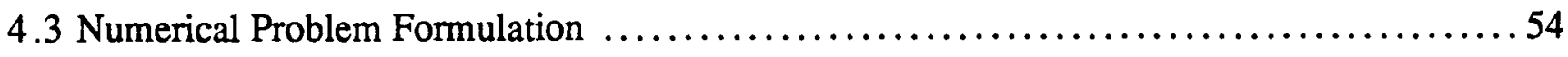

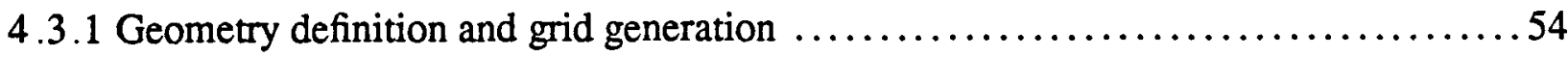

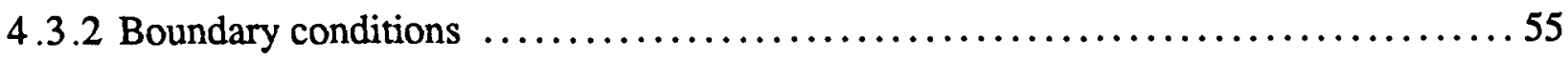

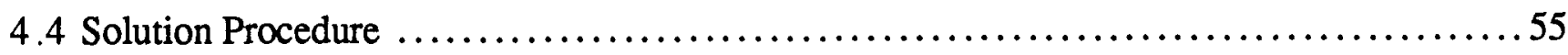

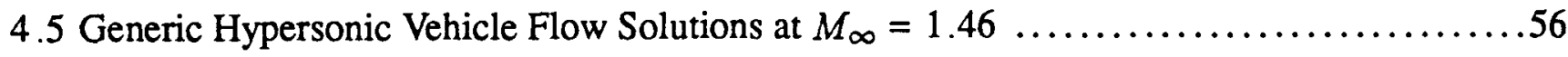

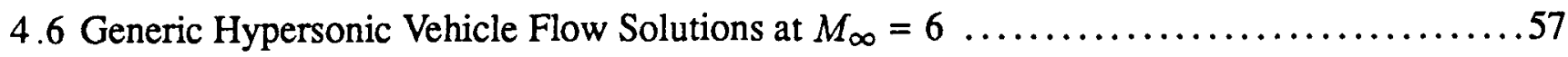

4.6.1 Analysis of the exhaust plume/afterbody interaction $\ldots \ldots \ldots \ldots \ldots \ldots \ldots \ldots \ldots \ldots . \ldots \ldots$ 
Chapter 5. CONCLUSION

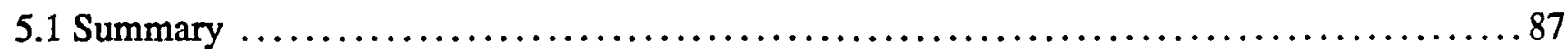

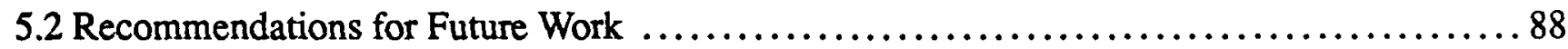

Appendix

SIMPLIFYING ASSUMPTIONS FOR THE

SPECIES CONTINUITY EQUATION

References 


\section{CHAPTER ONE}

\section{INTRODUCTION}

\subsection{Background}

Hypersonic flight is a vital link in the advancement of transportation within and beyond the earth's atmosphere. Whether it requires the relatively short exposure of a reentry vehicle, or the prolonged cruise of a hypersonic transport, surviving the aerothermodynamic environment characteristic of this flight regime constrains the design of a wide class of aerospace vehicles. This investigation studies the details of an important determinant of one type of hypersonic vehicle's performance: impingement of the engine exhaust plume on the underbody. In order to appreciate the parameters governing the design of a hypersonic vehicle, it is helpful first to classify the hypersonic flight condition.

The boundary between "supersonic" and "hypersonic" flight is somewhat vague. Certainly not less than Mach 3, nor greater than Mach 10, the dividing line defies straightforward determination of the point at which flight conditions are first considered hypersonic. In fact, the distinction is more correctly associated with the energy of the approaching air (with respect to the reference frame moving with the flight vehicle). Because the air is brought to a rest at the surface of the vehicle, the stagnation energy provides a meaningful measure of the thermal environment surrounding the aircraft. For an ideal gas, the stagnation energy has a quadratic dependence on the flight Mach number. Thus, at subsonic speeds, the stagnation energy is a small percentage above the static energy, while at supersonic speeds, the stagnation energy rises rapidly. In the Mach 3 to Mach 10 interval, the stagnation energy increases from a level about double the static energy to nearly ten times that level. The emergence in this interval of flow energy as a prominent feature sets hypersonic flow apart from supersonic.

Whereas pressure loads drive the design and analysis of supersonic vehicles, high-temperature phenomena assume central importance for hypersonic flight. As the flight Mach number increases, strong shocks can energize the air sufficiently to cause excitation of new internal energy modes and enable chemical reactions among the component species of air. These phenomena, inconsistent with ideal gas assumptions, significantly alter the shock structure, temperature distribution, and transport properties of air from the results of an ideal gas analysis. Hypersonic aerodynamics, then, is not simply an extension of supersonic aerodynamic theory; rather, it is a physical science unto its own. Development of a hypersonic flight vehicle requires new technology in materials, thermal protection, aerodynamic design and propulsion to address the constraints attendant to the hypersonic flight regime [1].

\subsection{Motivation}

Research stimulated by federally-sponsored programs such as the Space Shuttle, the National Aerospace Plane (NASP), the Aero-assisted Transfer Vehicle (AOTV) and the Aero-assisted Flight Experiment 
(AFE) has given rise to technological advancements in several critical areas. Experts now suggest that sustained hypersonic flight is not only possible, but also practical $[2,3]$. The promise of reducing trans-Pacific flights from a grueling 16 hours to about 2 hours in a Mach 14 airliner has piqued commercial interest. Furthermore, airbreathing scramjet engines, which benefit payload capacity dramatically by eliminating the need to carry oxidizer, make numerous aerospace missions feasible. However, before any of these goals are realized, many technological questions remain to be answered.

\subsection{Problem Definition}

Propulsion is the primary technical problem in designing an air-breathing hypersonic vehicle [4]. At a cruise speed in excess of Mach 6, few practical power plant choices exist. The hydrogen-powered scramjet engine will likely be employed for the first generation of hypersonic vehicles. As suggested by the name, the scramjet (supersonic-combustion ramjet) maintains supersonic flow through the combustion chamber. The hydrogen fuel mixes and burns rapidly enough with atmospheric oxygen to contain the process within a reasonably-sized engine. However, scramjets can operate only at flight Mach numbers above about 4, so alternate propulsion systems are required to accelerate the vehicle from takeoff to cruise speed. Undoubtedly, the hypersonic cruise vehicle will require multiple propulsion systems, for example, an afterburning turbojet for acceleration from rest to low supersonic speed, a ramjet for supersonic acceleration, and finally a scramjet for cruise [5].

Aside from the obvious logistical problems of installing and operating several engines on the same vehicle, researchers have yet to prove conclusively that an installed scramjet can produce net thrust. For an airbreathing engine, net thrust represents the difference between gross nozzle thrust and inlet ram drag. Preliminary studies indicate that a Mach 12 hypersonic cruise vehicle weighing $500,000 \mathrm{lb}$ could experience ram drag as high as 1.95 million pounds, requiring up to 2.1 million pounds of gross thrust [6]. The thrust margin, or percentage of gross thrust in excess of ram drag, in this instance is only $7.7 \%$. Needless to say, small deviations from design conditions could easily lead to inadequate engine performance.

Maximizing thrust and minimizing drag necessitates using the entire underbody to process the mass flow through the engine. Such an "airframe-integrated" propulsion system takes advantage of the precompressed forebody flow upstream of the engine inlet. Ideally, the inlet captures only air that has traversed the vehicle's bow shock. To design an inlet that captured air outside the bow shock would lead not only to unacceptably nonuniform inlet entrance conditions, but also to a severe inlet drag penalty attributed to the inlet "bow" shock. Upon entering the scramjet inlet, the precompressed capture air is further compressed in a series of ramps before reaching the combustion chamber. The combustion chamber consists simply of a diverging duct with fuel injectors installed in the sidewalls and the inlet struts. Hydrogen fuel possesses the advantage of a high flame speed, which reduces the length of the combustion chamber. However, it is also a comparatively low density fuel and hence constrains the vehicle size to accomodate a large fuel volume. How completely the fuel will be burned within the combustion chamber is unresolved.

Airframe integration will also come into play in expanding the exhaust gases. The high energy gas exiting the nozzle will be significantly higher than ambient pressure, but installing a nozzle to expand this gas completely is impractical. Not only would it be extremely heavy, but it would also create unnecessary drag due to the additional wetted area. In fact, very little of the exhaust gas expansion will be carried out internally. Instead, the exhaust gas will expand externally, washing the afterbody with an underexpanded jet 
flow. In this region, the flow will bear similarities to both a diverging nozzle flow and an underexpanded free jet. Careful contouring of the afterbody shape is critical to extract all the available thrust from the engine. Equally important, the high pressure gas impinging on the underbody will contribute to the lift and pitching moment of the vehicle, and these effects must be accounted for in locating the engine, sizing the wing planform, and assessing control surface requirements.

Clearly, design of the hypersonic vehicle's propulsion system will play a significant role in the overall design of the vehicle itself. A successful design requires detailed understanding of the flow physics throughout the propulsion system, along with the capability to predict how propulsion system performance is influenced by the geometry of the vehicle. A brief review of prior and ongoing work will help place the present work in perspective.

\subsection{Literature Review}

Integration of airframe and propulsion systems in transonic and supersonic flight has been an area of active research over the past decade. Investigations of inlet flows using Navier-Stokes computational fluid dynamics (CFD) codes have been performed for wing/nacelle and fuselage/inlet geometries, and mixed compression ramp inlets [7-9]. For a review of current literature on this subject, see reference [7]. A proliferation of work has also come forth recently concerning rocket and missile base flows at supersonic speeds. These include axisymmetric Euler methods [10], axisymmetric Navier-Stokes zonal methods [11], axisymmetric thin-layer and full Navier-Stokes methods [12], and three-dimensional NavierStokes methods [13]. However, in comparison to the deliberate progress seen in transonics and supersonics, research in hypersonics has experienced a more spasmodic history.

Spurred by the Apollo program and an ambitious planetary exploration agenda, the late 1960s and early 1970s saw rapid progress in hypersonics research. Researchers addressed the problem of scramjet propulsion by performing experiments on engine components in high-speed wind tunnels, and by applying proven analytical tools such as the method of characteristics and Newtonian flow theory [14-19]. But research activity declined sharply in the early 1970s, following the successful completion of the Apollo program and preliminary design of the Space Shuttle. Hypersonics research underwent a decade-long hiatus, brought to an end within the last few years by the technological requirements of a new generation of hypersonic flight vehicles.

In an effort to make up for lost time, many existing facilities are being brought out of mothballs, from shock tubes and ballistic ranges to hypersonic wind tunnels and arcjets. Because of the importance of this research to national security, only a few current experimental results in the hypersonic regime are available in open literature. Lockman [20] is presently studying the unclassified hypersonic all-body vehicle in the NASA Ames 3.5-foot hypersonic tunnel. Lockheed has tested a generic hypersonic vehicle at transonic and supersonic Mach numbers in a power-on simulation (private communication from D. Schuster, LockheedGeorgia Co., Marietta, Georgia). Regardless of the availability of such data, ground-based test facilities pose an overriding shortcoming: very few of them are capable of simulating the high altitudes, Mach numbers, and Reynolds numbers of actual flight conditions. Computational fluid dynamics is poised to supply that capability.

The past decade has been witness to a meteoric advancement in CFD capabilities. A synergistic coupling of improvements in computer resources and numerical solution algorithms has resulted in 
progressively faster, more accurate, less expensive flow solutions [21]. MacCormack's predictor-corrector method, now an industry standard, opened the door to Navier-Stokes calculations [22]. The advent of practical implicit methods, such as the Beam-Warming algorithm [23], cut computer run times by an order of magnitude or more, making complex three-dimensional flow solutions possible. By applying a diagonalization procedure to the Beam-Warming algorithm, Pulliam and Chaussee cut computer time by another order of magnitude [24]. The generality and efficiency of these algorithms has given rise to CFD research on problems with unprecedented geometrical and physical complexity. Numerous investigators have demonstrated flow solutions for viscous flow about realistic three-dimensional geometries at transonic and supersonic speeds $[9,25]$.

The biggest obstacle to extending these codes to solve hypersonic flows has been capturing the strong shock waves associated with the forward-facing surfaces. Development of flux-split [26] and totalvariation-diminishing (TVD) algorithms [27-30] has ameliorated this problem substantially, bringing forth a generation of robust codes capable of solving hypersonic flows [31-33]. These codes are now being applied to entire hypersonic vehicles and to specific components, mainly those associated with the propulsion system [34-38]. For the first time, problems are being attempted which model three-dimensional geometries and real gas flows, either in chemical equilibrium [39,40] or nonequilibrium [41]. At this time, it is appropriate to reach beyond code calibration analyses for these flow fields, and address performancerelated issues associated with scramjet propulsion.

\subsection{Objective}

The goal of the present work is to demonstrate a capability to solve complex nozzle/afterbody problems in hypersonics. It is first sought to calibrate the computational results with available experimental data, then to analyze the impingement of an underexpanded jet on the vehicle afterbody. This information will contribute to a predictive method for measuring installed scramjet performance, particularly as it relates to afterbody geometry and the interacting flow of external fluid and exhaust gases.

The following pages present an explanation of the theories, methods, and results associated with this investigation. First, the governing equations are presented with a description of their assumptions and limitations. The numerical methods employed to solve these equations are described, along with boundary conditions and the zonal grid methodology. Next, the method is applied to two test cases. First, a simplified nozzle/afterbody geometry is studied to gain an understanding of parametric influences on the plume/afterbody interaction. Then a more realistic generic hypersonic vehicle is analyzed to examine the effect of the exhaust plume on the aerodynamic forces of an entire body. Experimental data calibrate the numerical results wherever possible. Finally, some conclusions are drawn about this application of CFD to the analysis of hypersonic propulsion systems. 


\title{
CHAPTER TWO
}

\section{GOVERNING EQUATIONS AND}

\author{
NUMERICAL METHODS
}

\subsection{Introduction}

This chapter presents the partial differential equations governing fluid flow, the Navier-Stokes equations. The thin-layer approximation is invoked, and the equations are Reynolds-averaged to facilitate modeling turbulence. The equations are then transformed to generalized curvilinear coordinates to simplify the boundary conditions and enable efficient use of the computer.

Because some of the flowfields being considered are composed of binary gas mixtures, a species continuity equation is also included. In its most general form, this equation (only one equation is required to trace two species) includes terms related to convection, diffusion, production, and destruction of a species. However, neglecting the diffusion, production and destruction terms greatly simplifies solving this equation. A loosely-coupled approach is applied to solve the system of equations wherein the Navier-Stokes equations are solved simultaneously, then the species equation is solved as a separate, scalar equation. Information about the species concentrations is fed back to the Navier-Stokes equations through an equivalent- $\gamma$ approach, which is also described in this section.

An overview of the Osher TVD scheme implemented to solve the Navier-Stokes equations follows. This upwind scheme features enhanced stability over conventional central-differencing schemes, and is well suited to hypersonic applications. The algorithm has been implemented within a three-dimensional, zonal grid approach that is adapted to discontinuous geometrical variations, as occur at the exit plane of a scramjet nozzle. The chapter concludes with a discussion of the turbulence model as applied to the flow fields of interest here.

\subsection{Governing Partial Differential Equations}

2.2.1 Navier-Stokes equations-In high Reynolds number flows, viscous effects are confined to a thin layer near solid surfaces. Viscous derivatives in the stream and crossflow directions are negligibly small, while derivatives normal to solid surfaces dominate near-wall phenomena. Retaining only the viscous derivatives in the body-normal direction leads to the thin-layer approximation. Transforming the equations from Cartesian coordinates to generalized curvilinear coordinates allows solid surfaces to be mapped to constant coordinate surfaces, thereby allowing the thin-layer approximation to be invoked for arbitrarilyoriented surfaces. The thin-layer Navier-Stokes equations written in generalized curvilinear coordinates are

$$
\partial_{\tau} \widehat{Q}+\partial_{\xi} \widehat{E}+\partial_{\eta} \widehat{F}+\partial_{\zeta} \widehat{G}=R e^{-1} \partial_{\zeta} \widehat{S}
$$


where

$$
\begin{gathered}
\widehat{Q}=J^{-1}\left[\begin{array}{c}
\rho \\
\rho u \\
\rho v \\
\rho w \\
e
\end{array}\right], \widehat{E}=J^{-1}\left[\begin{array}{c}
\rho U \\
\rho u U+\xi_{x} p \\
\rho v U+\xi_{y} p \\
\rho w U+\xi_{z} p \\
U(e+p)-\xi_{t} p
\end{array}\right] \\
\widehat{F}=J^{-1}\left[\begin{array}{c}
\rho V \\
\rho u V+\eta_{x} p \\
\rho v V+\eta_{y} p \\
V(e+p)-\eta_{z} p
\end{array}\right], \widehat{G}=J^{-1}\left[\begin{array}{c}
\rho W W+\zeta_{x} p \\
\rho v W+\zeta_{y} p \\
\rho w W+\zeta_{z} p \\
W(e+p)-\zeta_{t} p
\end{array}\right]
\end{gathered}
$$

The vector of conservative variables $\widehat{Q}$ is composed of density $(\rho), x-, y$-, and $z$-momentum ( $\rho u, \rho v$, and $\rho w$, respectively), and total energy (e). The pressure is designated by $p$, and $\xi_{x}, \xi_{y}$, etc. represent the transformation metrics. $U, V$, and $W$ are the contravariant velocity components, defined as

$$
\begin{gathered}
U=\xi_{t}+\xi_{x} u+\xi_{y} v+\xi_{z} w \\
V=\eta_{t}+\eta_{x} u+\eta_{y} v+\eta_{z} w \\
W=\zeta_{t}+\zeta_{x} u+\zeta_{y} v+\zeta_{z} w
\end{gathered}
$$

The viscous flux terms are given by

$$
\widehat{S}=J^{-1}\left[\begin{array}{c}
0 \\
\mu m_{1} u_{\zeta}+(\mu / 3) m_{2} \zeta_{x} \\
\mu m_{1} v_{\zeta}+(\mu / 3) m_{2} \zeta_{y} \\
\mu m_{1} w_{\zeta}+(\mu / 3) m_{2} \zeta_{z} \\
\mu m_{1} m_{3}+(\mu / 3) m_{2}\left(\zeta_{x} u+\zeta_{y} v+\zeta_{z} w\right)
\end{array}\right]
$$

where

$$
\begin{aligned}
& m_{1}=\zeta_{x}^{2}+\zeta_{y}^{2}+\zeta_{z}^{2} \\
& m_{2}=\zeta_{x} u_{\zeta}+\zeta_{y} v_{\zeta}+\zeta_{z} w_{\zeta} \\
& m_{3}=\left(u^{2}+v^{2}+w^{2}\right) / 2+\operatorname{Pr}^{-1}(\gamma-1)^{-1}\left(a^{2}\right)_{\zeta}
\end{aligned}
$$

In the foregoing equations, density is nondimensionalized by $p_{\infty}$, Cartesian velocity components are nondimensionalized by $a_{\infty} / \sqrt{\gamma}$, and energy by $p_{\infty}$. Temperature, a derived quantity, is nondimensionalized by $T_{\infty}$. The Reynolds number is symbolized by $R e$, the Prandtl number by $P r$, and $\gamma$ represents the ratio of specific heats. The dynamic viscosity is symbolized by $\mu$.

Pressure is related to the conservative flow variables $\widehat{Q}$ by the ideal gas equation of state

$$
p=(\gamma-1)\left[e-\frac{1}{2} \rho\left(u^{2}+v^{2}+w^{2}\right)\right]
$$


The metric terms are defined as

$$
\begin{array}{ll}
\xi_{x}=J\left(y_{\eta} z_{\zeta}-y_{\zeta} z_{\eta}\right), & \eta_{x}=J\left(z_{\xi} y_{\zeta}-y_{\xi} z_{\zeta}\right) \\
\xi_{y}=J\left(z_{\eta} x_{\zeta}-z_{\zeta} x_{\eta}\right), & \eta_{y}=J\left(x_{\xi} z_{\zeta}-z_{\xi} x_{\zeta}\right) \\
\xi_{z}=J\left(x_{\eta} y_{\zeta}-y_{\eta} x_{\zeta}\right), & \eta_{z}=J\left(y_{\xi} x_{\zeta}-x_{\xi} y_{\zeta}\right) \\
\zeta_{x}=J\left(y_{\xi} z_{\eta}-z_{\xi} y_{\eta}\right), & \xi_{t}=-x_{\tau} \xi_{x}-y_{\tau} \xi_{y}-z_{\tau} \xi_{z} \\
\zeta_{y}=J\left(z_{\xi} x_{\eta}-x_{\xi} z_{\eta}\right), & \eta_{t}=-x_{\tau} \eta_{x}-y_{\tau} \eta_{y}-z_{\tau} \eta_{z} \\
\zeta_{z}=J\left(x_{\xi} y_{\eta}-y_{\xi} x_{\eta}\right), & \zeta_{t}=-x_{\tau} \zeta_{x}-y_{\tau} \zeta_{y}-z_{\tau} \zeta_{z}
\end{array}
$$

and

$$
J=\left[x_{\xi} y_{\eta} z_{\zeta}+x_{\zeta} y_{\xi} z_{\eta}+x_{\eta} y_{\zeta} z_{\xi}-x_{\xi} y_{\zeta} z_{\eta}-x_{\eta} y_{\xi} z_{\zeta}-x_{\zeta} y_{\eta} z_{\xi}\right]^{-1}
$$

is the independent variable transformation Jacobian.

2.2.2 Species continuity equation-Gas leaving the scramjet nozzles is highly energized, and consists of a mixture of fuel, combustion products, and the air that passes through the engine. Whereas the external flow is comprised primarily of molecular nitrogen $\left(\mathrm{N}_{2}\right)$ and oxygen $\left(\mathrm{O}_{2}\right)$, the nozzle flow of an airbreathing, hydrogen-powered scramjet will also contain water $\left(\mathrm{H}_{2} \mathrm{O}\right)$ and various intermediate reaction products. Indeed, for some flight conditions the flow will still be reacting as it leaves the nozzles. In any event, the exhaust flow will certainly possess markedly different thermodynamic properties than the external flow. These must be accounted for in the governing equations to properly simulate a scramjet plume flowfield.

As a first approximation, it is assumed that the chemical composition of the exhaust flow remains fixed after leaving the nozzle. This approximation models "frozen" flow, for which the convection time is very short compared to the chemical relaxation time. In other words, the exhaust gas traverses the body so fast that its composition effectively remains the same from the nozzle exit to the end of the body.

It is further assumed that the exhaust gases can be lumped together as a single species, and the external flow a second species. Thus, the flow consists of two streams of gases that have constant, but different, isentropic exponents: coflowing, dissimilar ideal gases. Accounting for mixing processes downstream of the nozzles requires determining the concentration of each species throughout the flow field. This is done by solving a species continuity equation. In its most general form, the species continuity equation is given by

$$
\frac{\partial\left(\rho c_{i}\right)}{\partial t}+\frac{\partial\left(\rho c_{i} u+j_{i_{x}}\right)}{\partial x}+\frac{\partial\left(\rho\left(c_{i} v+j_{i_{y}}\right)\right.}{\partial y}+\frac{\partial\left(\rho c_{i} w+j_{i_{z}}\right)}{\partial z}=K_{i}
$$

where $c_{i}=\rho_{i} / \rho$ is the mass concentration of species $i[42] . K_{i}$ is the net rate of production of species $i$ per unit volume, and $\left(j_{i_{x}}, j_{i_{y}}, j_{i_{z}}\right)$ is the diffusive mass flux vector defined by

$$
\mathbf{j}_{i}=\frac{n^{2}}{\rho} \sum_{j=1}^{\nu} m_{i} m_{j} D_{i j} \mathbf{d}_{j}-D_{i}^{T} \frac{\partial \ln \mathrm{T}}{\partial \mathbf{r}} \quad i=1,2,3, \cdots \nu
$$

$D_{i j}$ is the multicomponent diffusion coefficient, and $D_{i}^{T}$ is the thermal diffusion coefficient. The quantity $\mathbf{d}_{j}$ is the macroscopic gradient vector, given by

$$
\mathbf{d}_{j}=\frac{\partial\left(n_{j} / n\right)}{\partial \mathbf{r}}+\left(\frac{n_{j}}{n}-\frac{n_{j} m_{j}}{\rho}\right) \frac{\partial \ln p}{\partial \mathbf{r}}-\left(\frac{n_{j} m_{j}}{p \rho}\right)\left[\frac{\rho}{m_{j}} \mathbf{X}_{j}-\sum_{k=1}^{\nu} n_{k} \mathbf{X}_{k}\right]
$$


In the foregoing equations, the $n_{i}$ are the number densities of the species, and $n$ is the overall number density of the mixture. The $m_{i}$ are the species molecular weights, and $\mathbf{X}_{k}$ represents the external force vector acting on the molecules.

Inspecting these equations reveals that there are four distinct sources of diffusion: (1) diffusion due to a concentration gradient, (2) pressure diffusion, (3) diffusion due to external forces, and (4) thermal diffusion. When these equations are Reynolds-averaged, a fifth type of diffusion appears: an "apparent diffusion" arising from averaging the unsteady convective terms, analogous to the Reynolds stresses that appear in the momentum equations. The role of (1) and (4) were assessed by Ahtye [43], and later (2) and (3) by Howe and Sheaffer [44] for the stagnation region of reentry vehicles. The order of magnitude analysis of Howe and Sheaffer indicates that, away from shocks and outside the boundary layer, the diffusive terms are negligible compared to the convective terms in the species continuity equation. A more careful analysis is contained in the appendix, so for now it is sufficient to say that the cases under consideration here feature high flow velocities and comparatively low pressure and temperature gradients, therefore convection dominates the solution for species concentrations.

Eliminating the diffusion terms dramatically simplifies the species continuity equation. Also, by expanding the differentials, terms corresponding to the mass continuity equation can be omitted, leaving

$$
\frac{\partial c}{\partial t}+u \frac{\partial c}{\partial x}+v \frac{\partial c}{\partial y}+w \frac{\partial c}{\partial z}=0
$$

When this equation is transformed to generalized curvilinear coordinates, it is written as

$$
\frac{\partial c}{\partial \tau}+U \frac{\partial c}{\partial \xi}+V \frac{\partial c}{\partial \eta}+W \frac{\partial c}{\partial \zeta}=0
$$

where $\tau=t$, and $U, V$, and $W$ are the contravariant velocity components, defined in equation (2.3).

2.2.3 Coupling the equation sets- The species continuity equation is coupled to the Navier-Stokes equations through $\gamma$, the ratio of specific heats. While this quantity is constant for a thermally and calorically perfect gas, its value depends on the composition of the gas. Kinetic theory [45] predicts that the isentropic exponent of a gas is equal to $(n+2) / n$, where $n$ is the number of degrees of freedom of the constituent molecules. Gases made up of diatomic molecules, such as air, have an isentropic exponent of 1.4, corresponding to the five degrees of freedom (three translational modes and two rotational modes). Having determined the species concentrations by solving the species continuity equation, the equivalent $\gamma$, designated $\tilde{\gamma}$, is found from

$$
\tilde{\gamma}=\frac{c_{1} c_{p_{1}}+\left(1-c_{1}\right) c_{p_{2}}}{c_{1} c_{v_{1}}+\left(1-c_{1}\right) c_{v_{2}}}
$$

which amounts to the ratio of mass-averaged specific heats. The ideal gas equation of state remains unchanged except that $\gamma$ is replaced by $\tilde{\gamma}$ :

$$
p=(\tilde{\gamma}-1)\left(e-\frac{1}{2} \rho\left(u^{2}+v^{2}+w^{2}\right)\right)
$$

Now the numerical methods used to solve the Navier-Stokes equations and species continuity equation are discussed. 


\subsection{Numerical Methods}

This section outlines the numerical methods used to discretize the partial differential equations. The present work is an outgrowth of the UWIN code, which was developed by Rai as a time-dependent code compatible with space-marching (PNS) codes [32]. The UWIN code implements the Osher upwind TVD scheme in an iterative, flux-split, implicit algorithm to be described below [30]. Upwind schemes offer advantages over conventional central-difference schemes for problems in hypersonics. To capture strong shocks in hypersonic flow, central-difference schemes require large amounts of user-specified artificial smoothing, which tends to degrade the accuracy of the solution. On the other hand, smoothing appears inherently in upwind schemes, enabling them to capture very strong shocks without arbitrary parameters. Implementing TVD logic further enhances the scheme by preventing new extrema from appearing in the solution. TVD schemes have demonstrated the ability to capture shock waves and slip surfaces in one or two grid points without oscillations [46]. The species continuity equation is solved uncoupled from the Navier-Stokes equations in a fully implicit, upwind, approximately-factored algorithm.

2.3.1 Osher upwind scheme- A lengthy digression into the mathematical foundation of TVD in general, or the Osher scheme in particular, is not warranted here. Instead, the important features of the algorithm are described. The reader is recommended to references [27-30] for a deeper analysis. To begin, consider the Navier-Stokes equations in curvilinear coordinates, given in equation (2.1). Neglecting the viscous terms for the moment leaves

$$
\partial_{\tau} \widehat{Q}+\partial_{\xi} \widehat{E}+\partial_{\eta} \widehat{F}+\partial_{\zeta} \widehat{G}=0
$$

The implicit scheme is written

$$
\frac{\widehat{Q}^{n+1}-\widehat{Q}^{n}}{\Delta \tau}+\frac{\widehat{E}_{i+1 / 2}^{n+1}-\widehat{E}_{i-1 / 2}^{n+1}}{\Delta \xi}+\frac{\widehat{F}_{j+1 / 2}^{n+1}-\widehat{F}_{j-1 / 2}^{n+1}}{\Delta \eta}+\frac{\widehat{G}_{k+1 / 2}^{n+1}-\widehat{G}_{k-1 / 2}^{n+1}}{\Delta \zeta}=0
$$

In the above equation, unmodified indices have been omitted (e.g., $\widehat{E}_{i+1 / 2, j, k}$ is written $\widehat{E}_{i+1 / 2}$ ). The numerical fluxes are linearized and split according to the scheme of Steger and Warming [26], an example of which is

$$
\widehat{E}_{i+1 / 2}^{n+1}=\widehat{E}_{i+1 / 2}^{n}+A_{i}^{+} \Delta Q_{i}+A_{i+1}^{-} \Delta Q_{i+1}
$$

where

$$
A^{+}=\frac{\partial\left(E^{+}\right)}{\partial Q}, \quad A^{-}=\frac{\partial\left(E^{-}\right)}{\partial Q}
$$

Using one-sided forward $(\Delta)$ and backward $(\nabla)$ differences on the split fluxes results in

$$
\begin{array}{r}
{\left[I+\frac{\Delta \tau}{\Delta \xi}\left(\Delta_{\xi} A^{-}+\nabla_{\xi} A^{+}\right)\right]\left[I+\frac{\Delta \tau}{\Delta \eta}\left(\Delta_{\eta} B^{-}+\nabla_{\eta} B^{+}\right)\right]} \\
{\left[I+\frac{\Delta \tau}{\Delta \zeta}\left(\Delta_{\zeta} C^{-}+\nabla_{\zeta} C^{+}\right)\right] \Delta \widehat{Q}=} \\
-\Delta \tau\left[\frac{\hat{E}_{i+1 / 2}^{n}-\hat{E}_{i-1 / 2}^{n}}{\Delta \xi}+\frac{\widehat{F}_{j+1 / 2}^{n}-\hat{F}_{j-1 / 2}^{n}}{\Delta \eta}+\frac{\widehat{G}_{k+1 / 2}^{n}-\widehat{G}_{k-1 / 2}^{n}}{\Delta \zeta}\right]
\end{array}
$$


The numerical fluxes on the right hand side of equation (2.20) are evaluated with the Osher scheme, which for first-order spatial accuracy is given by

$$
\widehat{E}_{i+1 / 2}=\frac{1}{2}\left[\widehat{E}_{i}+\widehat{E}_{i+1}-\int_{Q_{i}}^{Q_{i+1}}\left\{\left\{\frac{\partial \widehat{E}}{\partial \widehat{Q}}\right\}^{+}-\left\{\frac{\partial \widehat{E}}{\partial \widehat{Q}}\right\}^{-}\right\} d \widehat{Q}\right]
$$

The integrals in the above equation are evaluated on three phase-space subpaths in the interval $(i, i+1)$. Let

$$
\Delta E^{+}=\int_{Q_{i}}^{Q_{i+1}}\left\{\frac{\partial \widehat{E}}{\partial \widehat{Q}}\right\}^{+} d \widehat{Q}
$$

Then the phase-space integrations are represented as

$$
\Delta \widehat{E}^{+}=\left(\Delta \widehat{E}^{+}\right)^{(1)}+\left(\Delta \widehat{E}^{+}\right)^{(2)}+\left(\Delta \widehat{E}^{+}\right)^{(3)}
$$

where

$$
\left(\Delta \widehat{E}^{+}\right)^{(j)}=k_{i+(j-1) / 3, i+j / 3}\left(\widehat{E}_{i+j / 3}-\widehat{E}_{i+(j-1) / 3}\right)
$$

and

$$
k_{i+(j-1) / 3, i+j / 3}=\left\{\begin{array}{l}
1, \text { if } \lambda_{i+(j-1) / 3}^{(j)}, \lambda_{i+j / 3}^{(j)} \geq 0 \\
0, \text { if } \lambda_{i+(j-1) / 3}^{(j)}, \lambda_{i+j / 3}^{(j)}<0
\end{array}\right.
$$

and $\lambda$ is the eigenvalue corresponding to the particular path. For a description of the second-order TVD scheme, see reference [30].

The Reimann invariants employed in the Osher scheme to solve equation (2.21) are derived for an ideal gas. The phase-space integrations in this equation are not exact for variable- $\gamma$ flows, and produced small oscillations in the solution variables in regions of concentration gradients. To ameliorate this problem, the isentropic exponent that appears in the Reimann invariants was Roe-averaged:

$$
\tilde{\gamma}_{i_{\text {ov }}}=\frac{\sqrt{\rho_{i} \boldsymbol{\gamma}_{i}}+\sqrt{\rho_{i+1} \gamma_{i+1}}}{\sqrt{\rho_{i}}+\sqrt{\rho_{i+1}}}
$$

This technique was found to reduce the magnitude of oscillations to less than $1 \%$ of the nominal value of the variables. The UWIN algorithm results in $5 \times 5$ block tridiagonal equations along the coordinate lines, which are solved by sweeping through the grid in each coordinate direction.

2.3.2 Upwind implicit algorithm for species continuity equation- The form of the species continuity equation solved in this study results in a scalar partial differential equation with constant coefficients. This type of equation is easily solved with an efficient and robust fully implicit algorithm. One-sided spatial differencing is applied according to the local flow velocity, and the equation is approximately factored so that it may be solved with scalar tridiagonal inversions in each of the three coordinate directions. Applying this method to equation (2.13) results in the finite difference equations

$$
\begin{aligned}
{\left[(\Delta t U) I+\delta_{\xi}^{u}\right] c^{\prime \prime} } & =c^{n} \\
{\left[(\Delta t V) I+\delta_{\eta}^{u}\right] c^{\prime} } & =c^{\prime \prime} \\
{\left[(\Delta t W) I+\delta_{\zeta}^{u}\right] c^{n+1} } & =c^{\prime}
\end{aligned}
$$


where $U, V$, and $W$ are the contravariant velocity components as defined in equation (2.3), and $\delta^{u}$ is an upwind difference defined as, for example,

$$
\delta_{\xi}^{u} c_{i}= \begin{cases}c_{i}-c_{i-1} & \text { if } U>0 \\ \frac{1}{2}\left(c_{i+1}-c_{i-1}\right) & \text { if } U=0 \\ c_{i+1}-c_{i} & \text { if } U<0\end{cases}
$$

\subsection{Turbulence Model Considerations}

Resolving the minute scales of turbulent flow requires an extremely fine grid, and gives rise to a wide range of time scales. The resultant stiff equation set would require a prohibitive amount of computer time under current computational capabilities. Therefore, modeling turbulence has become standard procedure for three-dimensional CFD problems. The algebraic eddy viscosity turbulence model of Baldwin and Lomax [47] is implemented in the UWIN code, and is unmodified for these solutions. This method replaces the molecular viscosity $\mu$ by an effective viscosity $\left(\mu+\mu_{T}\right)$ in the Navier-Stokes equations. Near the wall, the turbulent viscosity is calculated based on Prandtl mixing-length theory, and farther from the wall the calculation is based on the vorticity distribution. For a complete description of the Baldwin-Lomax turbulence model, see reference [47].

A few comments are appropriate regarding the use of this turbulence model for the present applications. First, the model has been shown to overpredict the turbulent viscosity in regions of moderate flow separation, causing the predicted separations to be smaller than those observed experimentally [48]. Also, the turbulent viscosity decays to zero far from the wall in this model. This means that the shear layer between the jet and external flow will develop only from the molecular viscosity, although laminar shear layers are known to become turbulent over a very short distance (lecture notes from B. J. Cantwell, Stanford University, Stanford, California). However, accurate prediction of turbulent shear layers requires a different turbulence model altogether: one that models turbulent viscosity in the absence of a wall. Since predicting shear layer development was not central to this work, no attempt was made to model them, and in any case, the grid point density away from the wall was insufficient to resolve a shear layer.

Finally, some of the cases presented in the following chapter involve coflowing streams of perfect gases. The Baldwin-Lomax turbulence model works equally well for both gases, but the constants that appear in the model were derived for constant pressure boundary layers at transonic speeds [47]. Corrections to these constants to account for hypersonic speed and gas mixtures are necessary, at a minimum, to properly account for the turbulent viscosity of the mixing gases. However, as a first approximation, it was assumed that the turbulent viscosity for both of the gases can be modeled with the Baldwin-Lomax turbulence model in its unmodified form. 


\section{CHAPTER THREE}

\section{ANALYSIS OF NOZZLE/AFTERBODY FLOW FIELDS}

\subsection{Introduction}

The aerodynamics of scramjet propulsion can be described as three distinct components: 1) forebody compression, 2) internal fuel injection and ignition, and 3) afterbody expansion (see figure 1). Compressing the flow on the forebody is accomplished when the oncoming air traverses the bow shock of the vehicle. This serves to create a dense, high pressure flow at the engine inlet, thereby eliminating the need for the internal compressors used in turbofan and turbojet engines. Needless to say, rotating compressors would be altogether impractical at hypersonic speeds, so external compression becomes a necessity. Ideally, forebody compression maximizes the mass flow at the inlet face and produces a uniform flow into the engine. The greater the mass flow into the engine, the more fuel can be burned, producing greater thrust. But approaching stoichiometric combustion of the fuel depends heavily on uniform flow conditions in the combustor. Scramjet inlet designers are challenged with producing a forebody and inlet shape that optimizes both mass flow and flow uniformity.

The internal segment of the scramjet propulsion cycle consists of further flow compression, followed by fuel injection and ignition. Injectors are placed on the sidewalls and struts so as not to block flow through the engine needlessly. Here, turbulent mixing processes will be relied upon to distribute the fuel efficiently, enabling rapid combustion. How well the fuel mixes with the ingested air is an important parameter governing scramjet performance. But the combustion kinetics of hydrogen and atmospheric oxygen in a supersonic stream represents a primary question being investigated by scramjet designers: how completely will the fuel be burned within the combustor, and what will the flow properties be at the nozzle exit plane? Hydrogen's high flame speed acts in favor of more complete combustion internally, and indeed, that is the main reason it is chosen over higher density fuels. However, at the upper limit of the flight envelope, say Mach 25 at 150,000 feet, the fuel will doubtlessly be only partly burned by the time it leaves the nozzle. Furthermore, much of the energy released during combustion will be absorbed by endothermic dissociation reactions of the combustion product $\left(\mathrm{H}_{2} \mathrm{O}\right)$. Recovering the energy sunk into dissociation reactions depends heavily on catalyzing recombination reactions in the exhaust flow. Attention then shifts to the reacting exhaust gas as it flows over the afterbody.

The final stage of the scramjet cycle, arguably the most important to its performance, is the flow of the underexpanded exhaust gases leaving the nozzle. Consider a scramjet-driven vehicle's afterbody, enclosed by a control volume as shown in figure 2. Neglecting upstream influence of the exhaust gas, the force on the body resulting from the exhaust gas is given by

$$
\mathbf{F}=-\int_{S_{1}} \rho \mathbf{V} \mathbf{V} \cdot d \mathbf{A}-\int_{S_{1}} p \hat{\mathbf{n}} \widehat{\mathbf{n}} \cdot d \mathbf{A}+\int_{S_{2}} \sigma_{i j} \cdot d \mathbf{A}
$$

In the above equation, $\mathbf{F}$ is the gross thrust, $d \mathbf{A}$ is an area element with outward-facing normal, and $\sigma_{i j}$ is the fluid stress tensor at the wall. Equation (3.1) reveals that gross thrust for the scramjet derives from 
two sources: 1) the pressure and momentum flux at the nozzle exit plane itself $\left(S_{1}\right)$, and 2$)$ forces arising from fluid stress acting on the surface of the afterbody $\left(S_{2}\right)$. The fluid stress at the wall consists of viscous shear stress acting parallel to the wall, and pressure acting normal to the wall. The shear stresses, which are realized as skin friction, mainly contribute to drag. Pressure, acting normal to the wall, produces lift, thrust, or drag, depending on the orientation of the surface. By incorporating an upswept afterbody, the high pressure exhaust gas generates both lift and thrust. A nose-down pitching moment also results from afterbody lift. Since propulsion system lift, thrust and moment account for a significant fraction of the overall forces on the hypersonic vehicle, effectively utilizing the afterbody will play an important role in obtaining the requisite propulsion system performance. The afterbody design, even the materials chosen to construct the airframe, will determine whether the energy in the fluid (kinetic, thermal, and chemical) is extracted to generate additional propulsive forces, or is left behind in the wake.

This chapter opens the investigation into exhaust plume/afterbody flow fields by analyzing a simplified geometry, the nozzle/afterbody model. To begin, the model and experimental procedure are described. A discussion of grid generation for the numerical problem follows, along with a description of the zonal grid scheme. Boundary conditions for this geometry are outlined next. To assess the computational effort involved in obtaining the solutions, the solution methodology and code performance are enumerated. Finally, numerical solutions are presented for the nozzle/afterbody flow fields. The sensitivity of afterbody forces to various parameters is assessed and explained based on the results. The chapter concludes with a grid refinement study for the nozzle/afterbody geometry.

\subsection{The Nozzle/Afterbody Model}

Over the past two decades, computational fluid dynamics has advanced rapidly from a fledgling analytical tool to a mature, multidisciplinary science. Applications of CFD to subsonic and transonic problems are diverse and well validated. Practical aerodynamic research in these flight regimes has made a place for CFD to play an important role in the preliminary design of aircraft components such as wings, control surfaces, and wing-nacelle fairings [8]. However, expanding the "envelope" of CFD capabilities to a new class of problems necessitates validating and/or calibrating the method against experimental or flight test data, or at least a well-proven, previously developed analytical method. Doing so instills confidence that the numerical model is rendering the correct representation of a physical problem. Calibration serves to modify assumptions in the numerical model that may no longer be valid for a new problem. In any case, applying a CFD method to one or more representative experiments enables the researcher to: 1) establish the capability of the code to solve a new class of problems, and 2) expand the information set associated with the experiment, thereby bringing forth a deeper understanding of the results.

Because this investigation does in fact address flight conditions heretofore unattempted by CFD codes, an important first step was to verify the accuracy of the numerical results by comparison with available experimental data. To this end, two experiments were identified as appropriate test cases. The first, a simplified nozzle/afterbody geometry, is the topic of this chapter. The relative simplicity of this model enabled parametric variations to be implemented and tested easily, some of which are addressed here.

3.2.1 Experimental apparatus and flow conditions- The experimental model under consideration here was analyzed by Cubbage and Kirkham [14] in the late 1960s, and had as its goal the measurement of the lift, drag, and pitching-moment increments resulting from the impingement of an underexpanded 
exhaust plume on a flat plate. The model, pictured in figure 3, consists of an inlet/nozzle assembly attached to a flat plate that represents a simplified hypersonic vehicle underbody. The plate is hinged at the nozzle exit plane to simulate varying afterbody upsweep angles. The afterbody portion of the model measures $17.78 \mathrm{~cm}$ long by $25.4 \mathrm{~cm}$ wide. The nozzle assembly is $9.14 \mathrm{~cm}$ wide and $2.29 \mathrm{~cm}$ high. Boundary layer trips were placed approximately $3 \mathrm{~cm}$ downstream of the leading edge to insure turbulent flow over the rest of the body. The tests were conducted in the Langley 20-inch Mach 6 tunnel at a stagnation pressure of $25 \mathrm{~atm}$ and a stagnation temperature of $480 \mathrm{~K}$. The Reynolds number was $20.9 \times 10^{6}$ per meter. Pressure taps were placed in a grid pattern downstream of the nozzle assembly in the jet expansion region.

3.2.2 Experimental procedure-Two axisymmetric nozzle geometries were tested with the flat plate model, one of which was designed for air $(\gamma=1.4)$, and the other for a gas mixture of $75 \%$ sulfur hexaflouride $\left(\mathrm{SF}_{6}\right)$ and $25 \%$ nitrogen $\left(\mathrm{N}_{2}\right)$ by weight, so chosen to yield an isentropic exponent, $\gamma$, of 1.26 at the nozzle exit plane. This mixture was chosen because it is relatively inert and stable, producing a dense, energetic gas akin to frozen flow in an actual flight condition. The nozzles were designed to produce nearly the same momentum flux and Mach number at the nozzle exit plane for both gases, and both had an exit diameter of $2.03 \mathrm{~cm}$. A "two-dimensional," or slot nozzle was also tested with air as the exhaust gas. The width of the slot nozzle was the same as the width of the axisymmetric nozzle assembly, $9.14 \mathrm{~cm}$. The height of the slot nozzle was $1.52 \mathrm{~cm}$. Air flowing into the inlet of the nozzle assembly was exhausted outside the test section, while the nozzle flow was supplied by a high pressure reservoir. Pressure data were reported along the model centerline (between the two inner nozzles) and on the centerline of an outboard nozzle. Normal forces on the plate were computed by discrete integration of the pressure data. Measuring the power-off static pressure on the plate downstream of the exhaust nozzles determined the power-off normal force, with which the researchers were also able to calculate the incremental forces and moments on the plate (that is to say, the forces and moments in excess of the power-off values).

\subsection{Numerical Problem Formulation}

Rarely is an actual or proposed problem so simple that it can be modeled exactly in a computational analogy. Simplifying the flow physics is essential in light of current computational capabilities; the physical approximations made in this study were already discussed in the preceding chapter. In addition, seemingly simple geometrical shapes prove astonishingly difficult to model mathematically. In this instance, an otherwise trivial flat plate geometry was complicated by an assembly of round nozzles, aligned side by side, within a square template. To generate a body-conforming grid for such a configuration would have been a substantial project in itself. An alternative method that modeled the nozzle assembly with a nonconforming grid was found to work quite satisfactorily. This section describes the geometry definition, grid generation, and boundary conditions that rendered the numerical analog to the nozzle/afterbody model.

3.3.1 Geometry definition and grid generation- The parametric variations performed in this experiment made it possible to assess the effect of afterbody upsweep, nozzle exit pressure ratio, exhaust gas isentropic exponent, and nozzle geometry on the forces and moments induced by the impinging exhaust plumes. Modeling the same effects computationally presented an opportunity to test the numerical method on a very practical application. Geometry definition for the nozzle/afterbody model simply amounted to defining a flat plate at arbitrary incidence. The geometry lent itself naturally to a Cartesian grid topology, and the three-dimensional grid was generated algebraically. Geometric stretching clustered grid points near the solid surfaces. The first grid point away from the plate surface was placed $0.001 \mathrm{~cm}$ away, which 
resulted in an average $y^{+}$of about 2.5. The quantity $y^{+}$, a nondimensional distance measured in wall units, is defined as

$$
y^{+}=\frac{\rho_{w} u_{\tau} y}{\mu_{w}}=\frac{\sqrt{\rho_{w} \tau_{w} y}}{\mu_{w}}
$$

where $u_{\tau}$ is the friction velocity, and $\tau_{w}$ is the wall shear stress. Wall units make a useful coordinate system to analyze turbulent boundary layers, and measuring the grid points in these units provides some insight into the grid resolution in terms of the flow variables. In order to make meaningful measurements of quantities such as skin friction and heat transfer, it is generally agreed that the normal grid spacing at the wall should be less than about ten wall units (private communication from D. S. Chaussee, NASA Ames Research Center, Moffett Field, California). Since the value of $y^{+}$is not known $a$ priori, experience is helpful in choosing appropriate clustering.

Figure 4 shows a perspective view of the grid topology, which was segmented into two zones. The two zones cannot be discerned in the figure because they are one-to-one at the interface. Zone 1 had 32 points in the stream direction, 33 points across the semispan, and 62 points in the plate-normal direction, while zone 2 had 31,33, and 62, respectively. Excluding the overlapping planes between zones, the grid totaled 122,760 points.

3.3.2 Zonal grid methodology-Dividing the spatial grid into two or more zones offers several advantages to solving complex flow problems. First, it permits the domain to be segmented into logical subunits, such as a forebody, wing, afterbody, and so on. Appropriate placement of zones also eliminates some grid singularities, such as discontinuous body cross sections. Finer grids can be used in multiple zone calculations than in single zone calculations if the computer's main memory limits the problem size, because only one zone resides in main memory at a time. Finally, the equation set can sometimes be simplified in some of the zones, thereby reducing the CPU time required to perform an iteration.

Several alternatives exist in developing a zonal grid scheme. The simplest is to impose one-to-one correspondence of the grid points at the zonal interface, as shown in figure 5. This approach enables direct flow field information transfer at each interface grid point, without interpolation whatsoever. While this approach is robust and easy to implement, it is not suited to geometrical discontinuities, which occur at the nozzle exit plane, for example. On the other hand, the most general zonal scheme permits arbitrary overlapping of grids without regard to the interface topology. A scheme with this capability has been developed for both two-dimensional and three-dimensional overlaid grids [49]. As yet, the transfer of flow field information in this scheme is nonconservative, so shock waves and other flow discontinuities may not propagate through zones correctly. Rai has developed and implemented a zonal scheme that permits planar zonal interfaces that need not be one-to-one [50]. Furthermore, the scheme ensures that the conserved quantities (mass, momentum, and energy) propagate through zones correctly.

For the present application, zonal grids were introduced primarily to reduce the main memory requirement. As such, a global grid was first generated about the entire geometry, then it was segmented into two zones along the body axis. These zonal interfaces are one-to-one, so no interpolation was necessary to pass information from one zone to the next. The zones were overlapped so that three redundant grid planes occurred at each interface, enabling boundary conditions at a zonal interface to be obtained from the interior of the adjacent zone. 
3.3.3 Boundary conditions- The solution domain includes only that part of the plate downstream of the exhaust nozzles. For the reasons mentioned earlier, the nozzle geometry was not modeled explicitly in the grid; rather, the effect of the nozzle flow (and surrounding solid surfaces) was accounted for through the inflow boundary conditions, as shown in figure 6. Specifically, a logical array was defined for the inflow plane that determined the type of boundary condition to apply at each point in that plane. Flow variables at points outside of the nozzle assembly region were fixed from a previously calculated turbulent flat plate solution. Flow variables at points within the nozzle assembly region of the inflow plane were specified to be either: 1) nozzle exhaust conditions, with all conservative variables fixed, or 2) solid surface conditions, with no slip and $\partial T / \partial n=0, \partial p / \partial n=0$. The temperature boundary condition, $\partial T / \partial n=0$, corresponds to an adiabatic wall condition, and is implemented in the code by specifying $T_{1}=T_{2}$. The pressure boundary condition corresponds to the normal momentum equation at the wall, and is implemented by specifying $p_{1}=p_{2}$. Both of these conditions are strictly correct only for a grid that is orthogonal at the wall. Having fixed the temperature and pressure at the wall, the conservative variables $\rho$ and $e$ were decoded using the equation of state.

Since the geometry and flow field are symmetric about the centerline of the model, only one half of the model was included in the domain. Symmetry boundary conditions were applied at the plate centerline: all conservative variables were reflected symmetrically acrosss the symmetry plane, except the y-momentum $(\rho v)$, which was reflected antisymetrically. Because the Osher scheme uses a five-point difference stencil, two reflection planes are required at the symmetry plane. Far field conditions were specified on the outer boundaries, in which all flow variables are set to their freestream values. At the outflow boundary, the conservative variables were linearly extrapolated from the points upstream. No-slip conditions were imposed at the plate surface, and turbulent flow was assumed from the inflow plane.

\subsection{Solution Procedure}

The conservative variables at interior grid points must be initialized to start the iterative solution algorithm. When no better initial guess was available, the flow variables were initialized to their freestream quantities. Since this "guess" of the solution is far from the converged result, a large perturbation occurs at the first time step. Stability bounds constrained the time step to a small value initially. It was then gradually increased as the solution progressed to the steady-state answer. Rather than fixing the time step explicitly, the UWIN code allows specification of the Courant number, defined as

$$
C N B R=\frac{\Delta t}{\lambda_{\max }}
$$

where $\lambda_{\max }$ is the largest eigenvalue of the flux Jacobian matrices. The time step is then calculated from the prescribed Courant number and the maximum eigenvalue. This method is convenient because the Courant number inherently accounts for the grid and flow features that affect stability. Based on experience with the UWIN code, the Courant number was initially set to a value of 0.1 . As the solution progressed, the Courant number was gradually increased to a maximum value of 100 . Although the code demonstrated stability at Courant numbers as high as 500 , generally no benefit to the convergence rate was observed at values greater than 100 .

Two criteria determined convergence: 1) the $L_{2}$ norm of the residual was reduced three orders of magnitude from its maximum value, and 2) the maximum change in density in one time step was less 
than $10^{-4}$. Upon satisfying both of these criteria, the Courant number was reduced to a value of unity and the solution was iterated further to eliminate high frequency errors. In practice, the axisymmetric nozzle cases did not satisfy the second convergence criterion. This is thought to be due to unsteadiness caused by periodic vortex shedding from the nozzle lip. Evaluation of the quasi-steady results indicate no demonstrable effect of the unsteadiness on the results presented here.

Solutions started from freestream conditions generally converged in about 600 iterations. Solutions were run on both the Cray X-MP and Cray 2; the code expends about 144 microseconds of CPU time per iteration per grid point on either. This equates to approximately 50 megaflops (million floating point operations per second) for the UWIN code. Total CPU time for a solution was about four hours.

To expedite convergence, in many instances new solutions were generated by perturbing converged solutions. For example, to calculate the flow solution at a new exit pressure ratio, the inflow boundary conditions were modified to reflect the new jet exhaust variables, and flow variables at the interior points were initialized from the previous solution. This procedure reduced run time by about $50 \%$, thereby enabling a more comprehensive analysis of parametric variations.

\subsection{The Nozzle/Afterbody Flow Field}

To facilitate later discussions, it is helpful first to describe the important features of an underexpanded jet flow, along with the peculiarities of the nozzle/afterbody flow field. A jet is termed underexpanded when the static pressure at the nozzle exit plane is greater than the ambient static pressure. Upon leaving the nozzle, the flow "expands" by turning toward the region of lower pressure, as shown in figure 7 . The adjacent supersonic external flow is forced to turn also, and an oblique shock wave (the plume shock) emanates from the nozzle lip. Meanwhile, an expansion fan propagates into the plume flow, eventually contacting and reflecting off of the afterbody surface. For two-dimensional and axisymmetric flows, waves continue to reflect off of the contact surface and nozzle centerline, giving rise to the diamond pattern sometimes seen in rocket plumes. For nonaxisymmetric geometries, waves propagate out of phase with one another. The ensuing nonsimple wave interactions dissipate the pressure gradients through fluid viscosity, so the wave structure is less apparent. The flow is yet more complex for the nozzle/afterbody model, as shown schematically in figure 8 , owing to the side-by-side axisymmetric nozzle configuration. The flow stagnates between adjacent nozzles. Expansion waves propagate into the circular jet flow from the entire circumference of the nozzle. And when adjacent nozzle plumes interfere with each other, new shocks form.

This flow pattern is also evident in the numerical solutions. For example, figure 9 shows the calculated pressure contours on the plate, at the symmetry plane of the model, and at the inflow plane. The freestream Mach number, $M_{\infty}$, for this solution is six, as for all the other nozzle/afterbody solutions presented. The angle of attack, $\alpha$, is five degrees, and the Reynolds number, Re, is $209,000 \mathrm{~cm}^{-1}$. The afterbody upsweep angle, designated by $\epsilon$, is zero degrees. The nozzle exit pressure ratio $\left(p_{e} / p_{\infty}\right)$ is 6.23 , and the exit Mach number $M_{e}$ is 3.5. At the inflow plane, pressure contours outline the nozzle circumferences. As described earlier, a Cartesian grid topology is employed with explicit inflow boundary conditions. The discrete representation of the round nozzles is evident from the pattern of pressure contours at the inflow plane. The plume shock is also readily evident in this view at the symmetry plane. Pressure peaks on the plate are apparent at the nozzle lip (where the exhaust flow first impinges on the plate), and further downstream where adjacent plumes first interact. 
Closer inspection of the flow near the nozzle exit reveals large regions of recirculating flow. A detailed view of the limiting streamlines near the plate for this case is shown in figure 10. By restricting these particles to remain in the coordinate surface nearest the plate, the path they trace is akin to an experimental oil flow visualization [51]. Between the nozzles, the flow stagnates for a distance of approximately one nozzle diameter downstream of the exit plane. At the downstream edge of this region, there exists a saddle point singularity where fluid particles are either entrained by the plume flow and carried downstream, or drawn into the eddying flow between the nozzles. The flow in the stagnant region then separates along a pair of separation lines, both of which terminate at the solid surface of the nozzle template. The postulated skin friction pattern for this flow is included in figure 10. According to rules of skin friction topology, the number of nodal points must exceed the number of saddle points by exactly two. As the figure indicates, the model features nine nodal points of attachment (one for each nozzle, one on each side of the nozzle assembly, and one in each aspiration zone), and one nodal point of separation (at the downstream boundary). Also shown are eight saddle points that result from the recirculating flow near the nozzle exit plane. Thus, the topological rule is satisfied and the interpretation is physically possible. However, closer analysis of this region may reveal yet more flow details. The pressure in the stagnant region is close to the ambient static pressure, and hence no lift or thrust is obtained here.

Surface pressure coefficients are compared with the experimental data for this case in figure 11. In figure 11(a), the pressure along the model centerline is seen first to rise due to a shock at $x / d \approx 1.5$, then fall to near ambient pressure at $x / d \approx 5$. The shock is the result of adjacent plumes interfering with one another as they expand to ambient pressure. The flow then overexpands, and at $x / d \approx 6, \mathrm{a}$ recompression is apparent. The comparison in this figure is generally good, though the strength of the first shock is underpredicted somewhat. This is due in part to the discrete modeling of the round nozzles, as will be shown later in a study of grid refinement: more accurate modeling of the nozzle results in greater interference and a higher pressure peak. Figure 11(b) shows the surface pressure coefficients on the centerline of the outboard nozzle. The pressure falls rapidly from its value at the exit plane, then reaches a plateau which occurs at $x / d \approx 1$ in the experimental data, and at $x / d \approx 2$ in the calculated result. The pressure plateau occurs where the expansion of the exhaust plume is restricted by interference with the adjacent plume. Downstream of this point, the flow becomes slightly overexpanded, then recovers to ambient pressure. Discrepancies are again attributed to grid resolution at the nozzle exit plane.

In the discussion above, reference was made to the high pressure region on the plate downstream of the nozzles. The term "footprint" is commonly used to describe the extent to which higher than ambient pressure prevails. As can be seen in figure 9, most of the normal force on the plate comes from the pressure peaks associated with the initial impingement of the exhaust fluid on the plate and, further downstream, the pressure peaks associated with mutual plume interference. These pressure peaks exhibit part of the net force-producing footprint on the afterbody. A useful parameter with which to compare afterbody forces is the normal force coefficient, designated $c_{n}$ and defined as

$$
\begin{aligned}
c_{n} & =\frac{\|\mathbf{N}\|}{\frac{1}{2} \rho_{\infty} V_{\infty}^{2} A_{\text {ref }}} \\
& =\frac{1}{\frac{1}{2} \gamma p_{\infty} M_{\infty}^{2} A_{\text {ref }}} \int_{A} p \widehat{\mathbf{n}} \cdot d \mathbf{A} \\
& =\frac{2}{\gamma M_{\infty}^{2} A_{\text {ref }}} \int_{A}\left(\frac{p}{p_{\infty}}\right) \hat{\mathbf{n}} \cdot d \mathbf{A}
\end{aligned}
$$


where $\mathbf{N}$ is the force normal to the afterbody, and $A_{\text {ref }}$ is the reference area of the plate $\left(452 \mathrm{~cm}^{2}\right)$. For this case, the normal force coefficient on the plate was computed to be 0.1256 . Since $\alpha=5^{\circ}$ and $\epsilon=0^{\circ}$ for this case, the afterbody force coefficients in Cartesian components are $c_{x}=0.0109$ and $c_{z}=0.1251$. The quantity $c_{n}$ will be useful later in correlating the calculations with experimental results.

\subsection{The Influence of Parametric Variations on Afterbody Forces}

Having gained some appreciation for the dynamics of underexpanded jet impingement, it is now of interest to investigate parametric variations of nozzle operating conditions. As parameters such as the exit pressure ratio, afterbody upsweep, and nozzle flow conditions change, the plume/afterbody interaction will be altered, impacting overall performance. Becoming familiar with these performance derivatives will be useful in interpreting later results.

3.6.1 Effect of nozzle exit pressure- Whereas turbojet engines derive most of their thrust by expanding the captured air to very high velocity inside the nozzle, scramjet engines must rely on external expansion of the fluid. A scramjet nozzle that completely expanded the exhaust gas could feasibly be larger than the vehicle it was designed to propel! Thus, fluid leaving the scramjet nozzle will be highly underexpanded. The nozzle exit pressure ratio - defined here as the ratio of static pressure at the nozzle exit plane to the freestream static pressure - is an important determinant of the afterbody forces. It influences the shape and force of the footprint the exhaust plume makes as it impinges on the afterbody, thereby contributing to the overall lift, thrust, and moment.

Solutions for the nozzle/afterbody model were obtained at an otherwise fixed operating condition with variations in exit pressure ratio. Comparisons of the surface pressure coefficient at two values of exit pressure ratio are shown in figure 12 for $\alpha=5^{\circ}, \epsilon=0^{\circ}$, and $p_{e} / p_{\infty}=2.07$ and 6.23 . The calculated results show good agreement with the data except in predicting the location of plume interference as noted earlier. Note that the $p_{e} / p_{\infty}=1$ data - the condition at which the nozzle flow is completely expanded - indicates that the flow initially overexpands because of low pressure in the aspiration region near the nozzle exit plane. Further downstream, recompression to ambient pressure is observed.

To assess the sensitivity of the afterbody normal force to the exit pressure ratio, solutions were obtained for $p_{e} / p_{\infty}=1,2,3$, and 4 at $\alpha=0^{\circ}, \epsilon=0^{\circ}$. Figure 13 presents incremental normal force coefficients as a function of the nozzle exit pressure ratio. To compute the "incremental" value, the experimentalists subtracted the power-off normal force coefficient, $c_{n_{0}}$, from the power-on value. The numerical solutions were correlated with this data by normalizing the calculations about $p_{e} / p_{\infty}=1$, thereby deducing $c_{n_{0}}$ as follows:

$$
\left(c_{n_{0}}\right)_{\text {numerical }}=\left[c_{n_{\text {mmerical }}}-\left(c_{n}-c_{n_{0}}\right)_{\text {experimental }}\right]_{p_{e} / p_{\infty}=1}
$$

The linear relationship between exit pressure and normal force is tentatively explained as follows: an initial expansion wave propagates into the plume with a slope determined only by the plume Mach number. In the region of exhaust flow undisturbed by expansion waves, the pressure is nearly the same as the exit pressure; thus, pressure on the plate will persist at this value until the first expansion wave reaches the surface. Thereafter, expansion proceeds until the ambient pressure is reached. Since the exit Mach number was held constant for these solutions, the expansion began at approximately the same location for 
all cases. Thus, as the exit pressure ratio was increased, the pressure in the basic footprint region rose proportionately, but the area over which it acted remained largely unchanged. The result was a linear increase in the normal force coefficient with exit pressure ratio.

This hypothesis is verified by comparisons of the footprint shape at each pressure ratio, shown in figure 14. The footprint shape is defined for this illustration by the location of the pressure contour on the plate that is $10 \%$ above freestream pressure. Hence, this contour envelops the region on the plate that produces force in excess of power-off conditions. At $p_{e} / p_{\infty}=3$ and 4 , the contours are very comparable, showing that the exit pressure has only a small influence on the size and shape of the footprint. At $p_{e} / p_{\infty}=2$, the footprint is considerably smaller, and at $p_{e} / p_{\infty}=1$, the footprint is virtually nil. However, these two lower values are so close to the ambient pressure even at the exit plane, that small pressure variations (arising from plume interference, for example) affect the footprint shape substantially.

3.6.2 Effect of afterbody upsweep-The increase in thrust derived from an upswept afterbody has already been discussed, though no mention was made of how much upsweep optimizes a design. The schematic in figure 15 shows that, on one hand, upsweep increases the overall footprint area of the exhaust plume. However, the flow expands in turning to follow the afterbody contour, so the footprint area on an upswept afterbody will exhibit lower pressure than the unswept case. Too much upsweep results in an overexpanded flow that is likely to separate from the body, reducing lift and increasing drag. Clearly, the optimal upsweep angle depends closely on the design exit pressure ratio.

Consider the surface pressure contour comparisons at $\alpha=0^{\circ}, p_{e} / p_{\infty}=3$ shown in figure 16. The footprint on the plate at $\epsilon=4^{\circ}$ extends to $x / d \approx 5$, whereas at $\epsilon=0^{\circ}$, it reaches only to $x / d \approx 4$. A twodimensional method of characteristics analysis predicted values of 5.1 and 4.2, respectively. Furthermore, because the upswept afterbody permits the exhaust flow to expand partially, the interference peaks for $\epsilon=4^{\circ}$ are not as high as the $\epsilon=0^{\circ}$ case. Table 1 below compares the forces exerted on the plate for this case and for $p_{e} / p_{\infty}=1$. One interesting observation from the table is that the incremental normal force is nearly the same for the flat and upswept afterbodies at a given exit pressure ratio. Apparently, the phenomena associated with upsweep are self-cancelling in these cases. The upswept afterbody's larger footprint tends to add to the plate force, while the added expansion decreases the pressure of the exhaust gas and tends to diminish the plate force. However, since the external flow must turn also, the ambient pressure drops along with the exhaust pressure. In fact, since the external flow is at $M_{\infty}=6$, PrandtlMeyer theory predicts that the external flow turning four degrees will experience a $59 \%$ drop in pressure; the exhaust flow, which is at $M_{e}=3.5$, will drop by only $36 \%$ [52]. The results in Table 1 suggest that the net effect is a slight loss in incremental normal force.

Table 1. Force coefficients for the flat and upswept afterbody. $M_{\infty}=6, \alpha=0^{\circ}$.

\begin{tabular}{|c|c|c|c|c|}
\hline$p_{e} / p_{\infty}$ & \multicolumn{2}{|c|}{1} & \multicolumn{2}{c|}{3} \\
\hline$\epsilon$ & $0^{\circ}$ & $4^{\circ}$ & $0^{\circ}$ & $4^{\circ}$ \\
\hline \hline$c_{x}$ & .0000 & .0013 & .0000 & .0021 \\
\hline$c_{z}$ & .0225 & .0190 & .0338 & .0296 \\
\hline$c_{n}$ & .0225 & .0190 & .0338 & .0297 \\
\hline
\end{tabular}


3.6.3 Effect of exhaust gas composition- At high altitude and Mach number flight conditions, scramjet exhaust species will continue to undergo chemical reactions after leaving the combustion chamber. However, if expansion occurs too rapidly, the temperature may drop below the threshold necessary for recombination reactions to proceed, freezing the composition in an intermediate state (private communication from G. S. Deiwert, NASA Ames Research Center, Moffett Field, California). When this happens, the exhaust flow traverses the body without reacting, behaving as an ideal gas that mixes with another ideal gas, the freestream air. By coupling a species continuity equation to the Navier-Stokes equations as described in chapter 2, it was possible to assess the effect that different (ideal) exhaust gases have on the afterbody forces. While there is no simplified theory to analyze such a flow, a heuristic argument is put forth here.

The experiment to which the present results are compared tested a mixture of $75 \%$ sulfur hexaflouride $\left(\mathrm{SF}_{6}\right)$ and $25 \%$ nitrogen $\left(\mathrm{N}_{2}\right)$, for which $\gamma_{e}=1.26$, exhausting into air $(\gamma=1.4)$. Kinetic theory [46] explains sulfur hexaflouride's lower value of $\gamma$ as the result of its more numerous modes of internal energy than those of air. At moderate temperatures, air, composed almost entirely of diatomic molecules, exhibits five modes of internal energy: three translational modes, and two rotational modes. The kinetic model predicts that $\gamma=(n+2) / n$, where $n$ is the number of internal energy modes. Thus, $\gamma_{a i r}=1.4$, as verified by observation. However, $\mathrm{SF}_{6}$ is a much larger molecule possessing numerous vibrational modes, many of which are fully excited at room temperature. This results in a lower value of $\gamma$. Internal energy must be distributed over many more modes in $\mathrm{SF}_{6}$ than air; therefore, $\mathrm{SF}_{6}$ has more internal energy than air at the same temperature. As the gas expands and cools, energy cascades out of the other internal energy modes and into kinetic energy. This will reveal itself in a smaller drop in pressure for a given volumetric expansion. Conversely, expanding from a fixed exit pressure to some lower pressure will require a greater volumetric expansion of $\gamma_{e}=1.26$ fluid than of $\gamma_{e}=1.4$ fluid. Hence, the plume footprint is expected to be larger, and the expansion more gradual for $\mathrm{SF}_{6}$ than for air.

Figure 17 bears out this argument. At $\alpha=0^{\circ}, \epsilon=0^{\circ}, p_{e} / p_{\infty}=4$, the footprint for $\mathrm{SF}_{6}$ exhaust gas is notably larger than for air: it persists an additional nozzle diameter downstream, and it spreads laterally by about another one-half nozzle diameter. Figure 18 shows pressure contours on the plate, demonstrating that the pressure within the $\mathrm{SF}_{6}$ footprint is higher as well; for this case, the incremental normal force for $\gamma_{e}=1.26$ was about $25 \%$ higher than for $\gamma_{e}=1.4$. Comparisons of the calculated and measured normal force coefficient at several exit pressure ratios are shown in figure 19. The variation of normal force coefficient with exit pressure ratio is still linear, but the slope is about $30 \%$ greater for $\gamma_{e}=1.26$ than for $\gamma_{e}=1.4$. Therefore, at the same exit conditions, a gas with a low value of $\gamma$ will produce greater afterbody forces than one with a high value of $\gamma$.

Contours of the exhaust gas species concentration are shown in figure 20. Since convection is the only transport mechanism modeled, the contours retain the general shape of the contours at the nozzle exit plane. They distort somewhat due to the expansion of the plumes, but the most significant species transport is seen to occur near the plate. In the boundary layer near the surface, lateral spreading is markedly enhanced because the velocity is so much smaller than the flow outside the boundary layer. The crossflow velocity becomes the same order of magnitude as the streamwise velocity, and exhaust gas species are thus convected much further laterally in the boundary layer than outside of it.

3.6.4 Effect of nozzle geometry-In figure 10, it was shown that adjacent axisymmetric nozzles leave regions of stagnant flow near the exit plane. This observation begs the question, "does a nozzle geometry 
that produces a 'cleaner' flow field near the exit generate greater afterbody forces?" To investigate this question, an alternate "slot" nozzle was tested against the axisymmetric nozzle geometry. The dimensions of the slot nozzle model were determined by specifying the overall width of the nozzle assembly to remain the same. The nozzle height was then fixed so as to produce the same momentum flux through the slot nozzle as the four axisymmetric nozzles at the same exit conditions. Test conditions for the comparison were $\alpha=5^{\circ}, \epsilon=0^{\circ}$, and $p_{e} / p_{\infty}=6.23$.

Comparisons of the calculated surface pressure coefficients with experimental data are shown in figure 21. Centerline pressure for the slot nozzle is seen to be consistently underpredicted from $x / d=0$ to $x / d \approx 5$. This is thought to be a result of two discrepancies between the experiment and the numerical model: 1) nonuniform experimental exit flow conditions, causing a pressure peak near the exit face, and 2) geometrical disparity, causing the predicted onset of expansion to occur about one nozzle diameter upstream of the experimentally determined location.

The flow pattern near the plate renders a clearer visualization of why the two nozzle geometries perform differently. Figure 22 presents limiting streamlines for the two nozzle geometries. These streamlines are colored according to the local static pressure, adding insight as to how the two flow fields generate plate forces. The slot nozzle produces a virtually two-dimensional flowfield away from the edges of the nozzle assembly. The flow is, indeed, much "cleaner" than the axisymmetric geometry, showing no recirculating flow near the nozzle exit. The payoff is in the afterbody normal force: $c_{n_{\text {stot }}}=0.0778$, while

$c_{n_{\mathrm{uxi}}}=0.0409$. The recirculation regions on the axisymmetric geometry dissipate nearly $50 \%$ of the incremental normal force compared to the slot nozzle! As suggested by figure 22, most of the additional force is generated where the axisymmetric nozzle flow creates low pressure recirculation regions.

\subsection{The Effect of Grid Resolution on Computed Results}

As a necessary final step to the nozzle/afterbody investigation, the convergence of the numerical results is tested on a series of grids. Any finite-difference method carries with it truncation errors arising from approximations to the differentiations. Furthermore, it can be shown that the truncation error is proportional to the cell volume (lecture notes from H. Lomax, NASA Ames Research Center, Moffett Field, California). Hence, as the grid is refined, the cell volume decreases, and truncation errors diminish. Obtaining numerical solutions on a series of grids tests the accuracy and uniqueness of the results. The value of some relevant flow field variable should approach a limit asymptotically as the grid is refined. This section presents the results of a grid refinement study performed for the nozzle/afterbody model at $\alpha=5^{\circ}, \epsilon=0^{\circ}$, and $p_{e} / p_{\infty}=6.23$.

To minimize the effects of unequal grid stretching on the results of this study, results were first obtained on a fine grid, which was then coarsened twice by discarding alternate grid points in each of the three coordinate directions. Though this technique does not preserve identical stretching on the coarsened grid, it does produce proportionately similar grid cells from one grid to the next. Discarding alternate grid cells in all three directions results in an eight-fold reduction in the total number of grid points. The fine grid dimensions were 119 points in the streamwise direction, 63 in the spanwise direction, and 123 in the platenormal direction, for an overall total of 922,131 points. Hence, the medium grid dimensions were 60,33 , and 62 points, respectively, for a total of 122,760 points; the coarse grid had 31,17 , and 31 for 16,337 . 
Pressure coefficient comparisons among solutions on the three grids are shown in figure 23. The coarse grid result follows the broad trends in the data, but fails to place the interference peak correctly in figure 23(a), and shows no evidence of recompression at $x / d=6$. The medium and fine grid results are nearly identical to one another, the only noteworthy difference being that the fine grid result shows a higher interference peak (figure 23(a)), with a curious saddle profile. Figure 23(b) shows similar trends.

Figure 24 compares boundary layer profiles among the solutions. The profiles between adjacent nozzles, figure 24(a), shows some differences in the solutions. The coarse grid predicts attached flow at this station, while the medium and fine grids show significant reverse flow. The flow reversal is precipitated by shock waves from the interaction of adjacent plumes, and the discrepancies seen in this figure are most likely a result of how well the plume interaction process is resolved. On the nozzle centerline, figure 24(b), all the results are identical, indicating that even the coarse grid adequately models the attached flow. To resolve the viscosity-dominated flow field near the nozzle exit would likely require a grid much finer than those studied here.

However, plate force measurements would not be sensitive to further refinement of the grid. Plate normal force coefficients, shown in Table 2, indicate an asymptotic trend toward a value of about 0.138 . The medium grid dimensions, which were used for the other nozzle/afterbody grids as well, appear to resolve most of the flow characteristics adequately, and can be used to calculate integrated quantities to within about $10 \%$ of the asymptotic value.

Table 2. Effect of grid density on plate normal force.

$M_{\infty}=6, \alpha=5^{\circ}, \epsilon=0^{\circ}, p_{e} / p_{\infty}=6.23$.

\begin{tabular}{|l|r|r|c|}
\hline Grid & Dimensions & Total Points & $c_{n}$ \\
\hline \hline Coarse & $31 \cdot 17 \cdot 31$ & 16,337 & 0.1009 \\
\hline Medium & $60 \cdot 33 \cdot 62$ & 122,760 & 0.1256 \\
\hline Fine & $119 \cdot 63 \cdot 123$ & 922,131 & 0.1362 \\
\hline
\end{tabular}

\subsection{Summary}

The nozzle/afterbody model has provided a reasonable idealization of the flow about an actual vehicle afterbody. By restricting the domain to only the region of interest, computational requirements were reduced enough to permit solutions for several parametric variations. Numerical results compared favorably with the experimental data, and inadequate resolution of the nozzle geometry may be the principal source of discrepancies. Having calibrated the code and gained an understanding of the exhaust plume/afterbody flow field, attention is now turned to the effect of the exhaust plume on a realistic hypersonic vehicle configuration. 


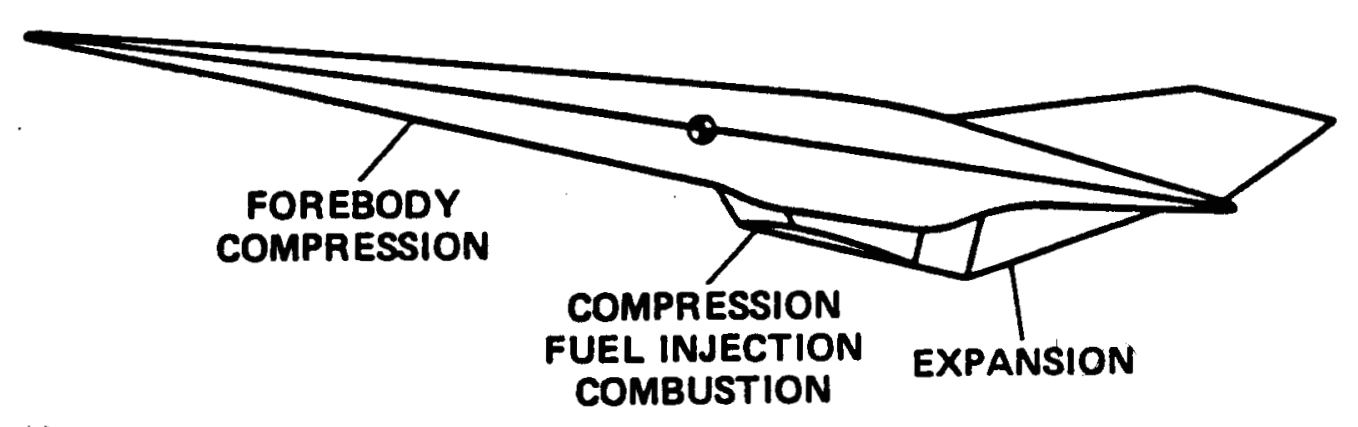

Figure 1. Schematic representation of the scramjet propulsion cycle. 


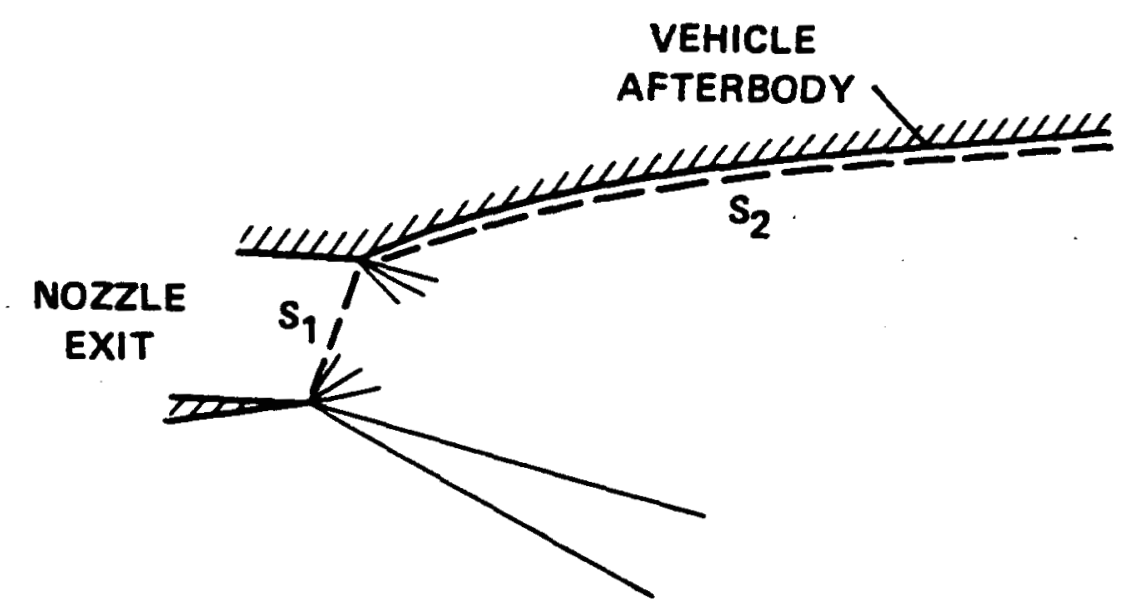

Figure 2. Control volume of nozzle and afterbody for force calculations. $S_{1}$ is the nozzle exit face, $S_{2}$ is the afterbody surface. 


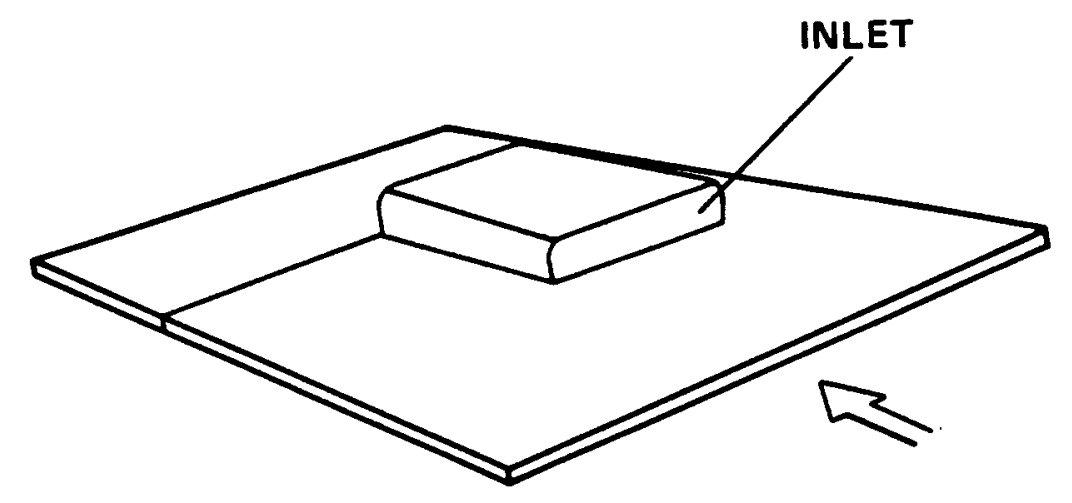

(a) front perspective

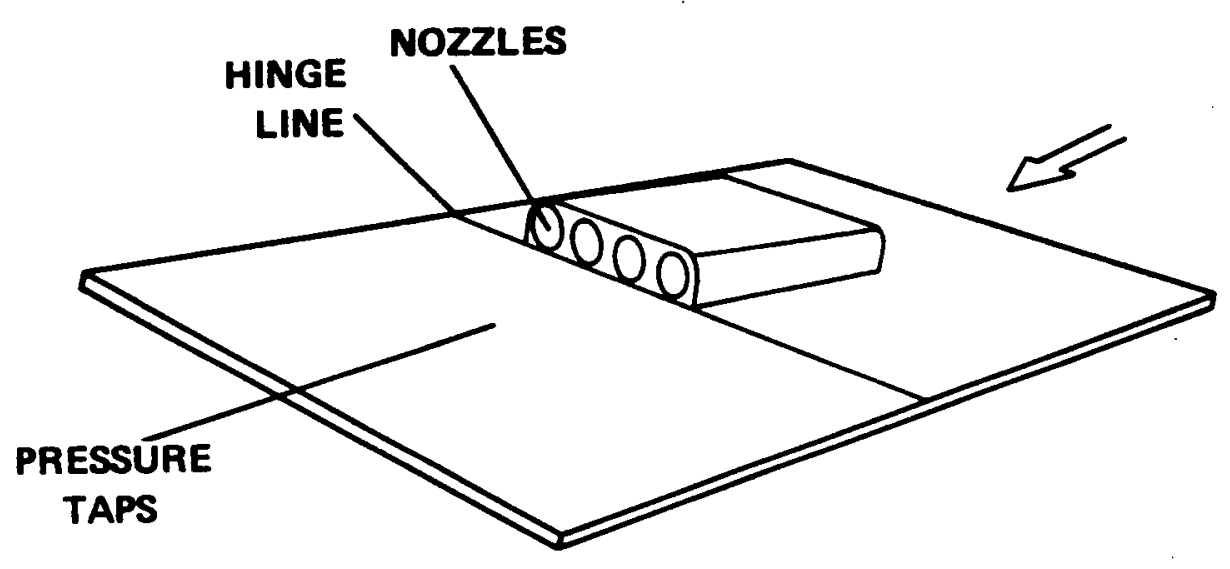

(b) rear perspective

Figure 3. Nozzle/afterbody model geometry. 


\section{ORIGINAL PAGE IS \\ OF. POOR QUALITY}

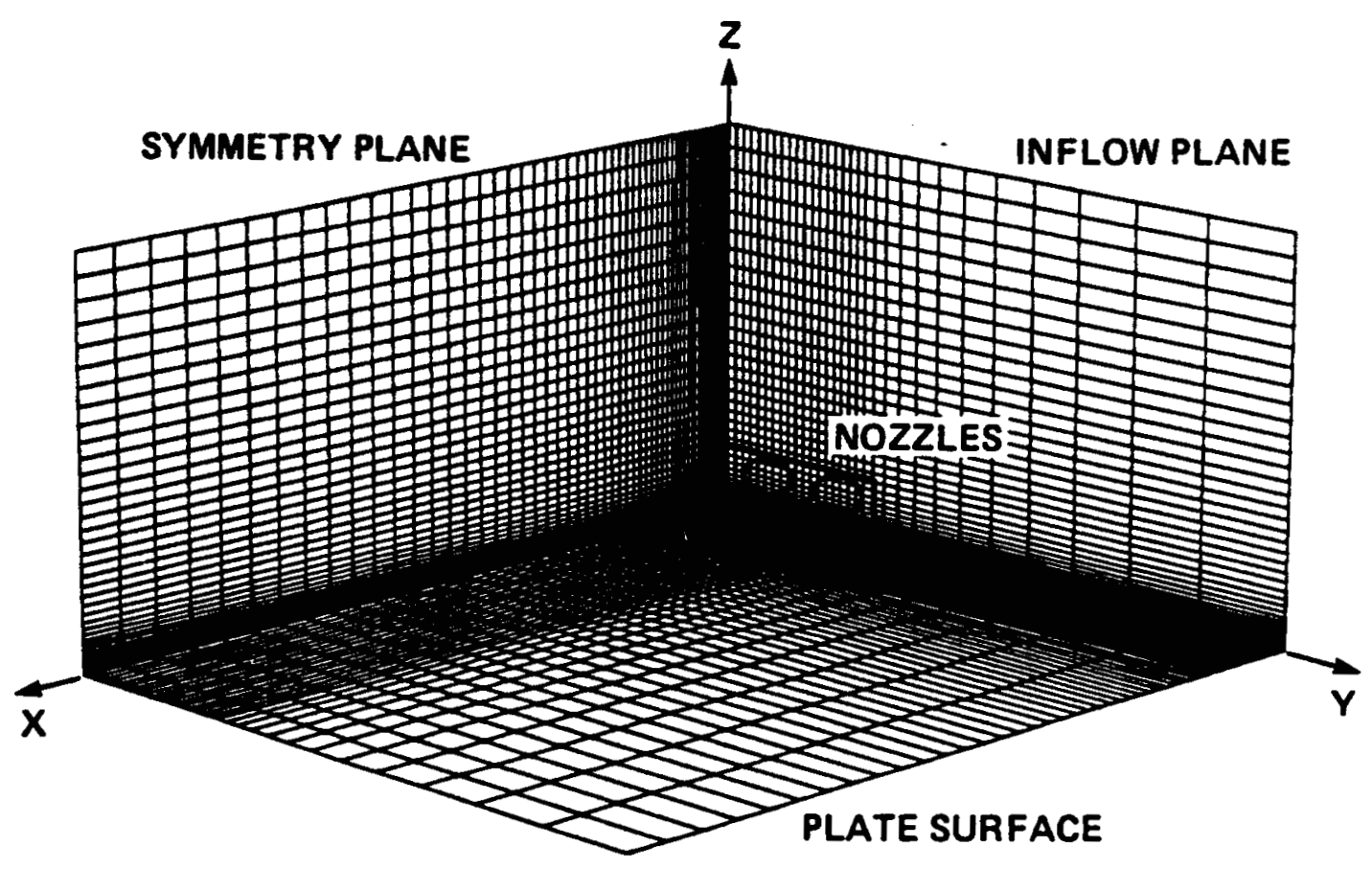

Figure 4. Cartesian grid topology for nozzle/afterbody model. 


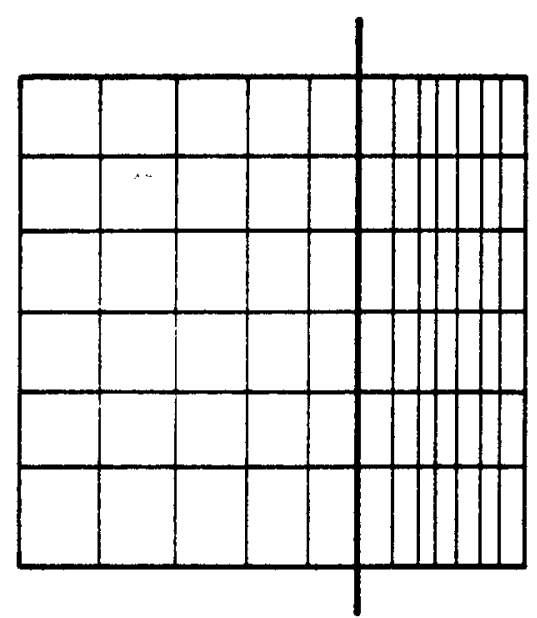

(a) one-to-one

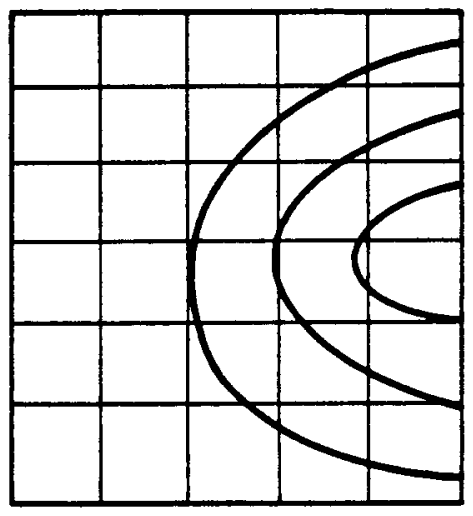

(b) overlaid

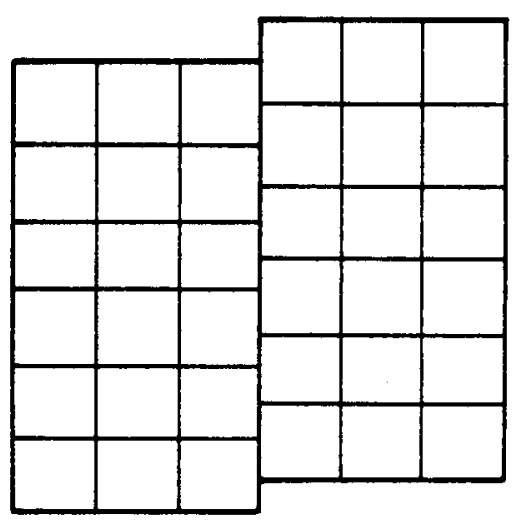

(c) patched

Figure 5. Types of zonal grid interfaces. 


\section{INFLOW BOUNDARY CONDITION \\ - TURBULENT FLAT PLATE SOLUTION \\ $\triangle$ SOLID SURFACE BOUNDARY CONDITIONS \\ O JET EXHAUST CONDITIONS}

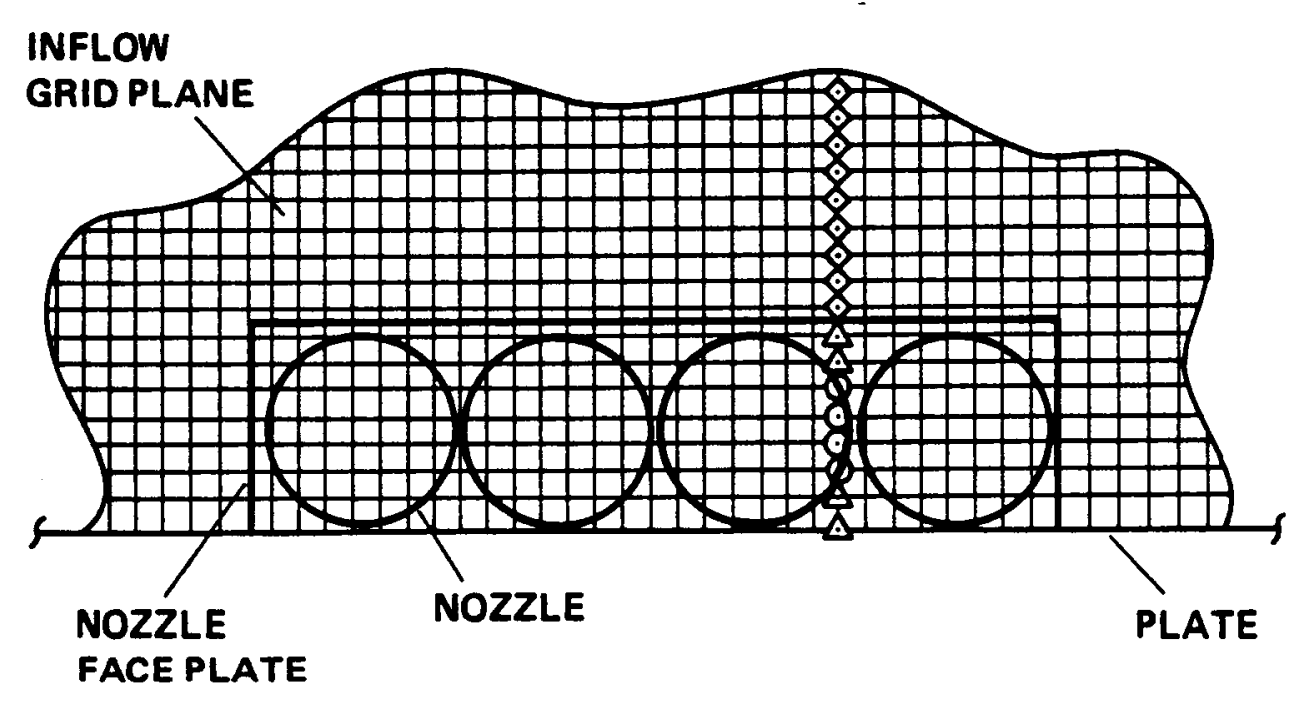

Figure 6. Inflow boundary conditions for nozzle/afterbody model. 


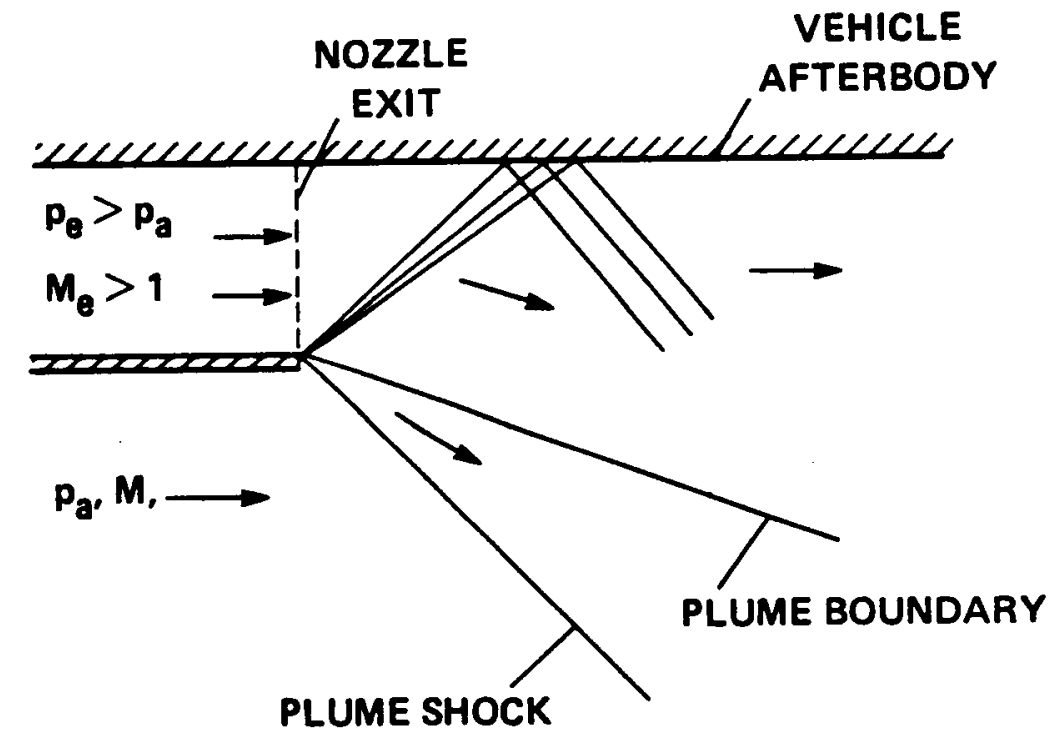

Figure 7. Schematic of an underexpanded jet flow field. 


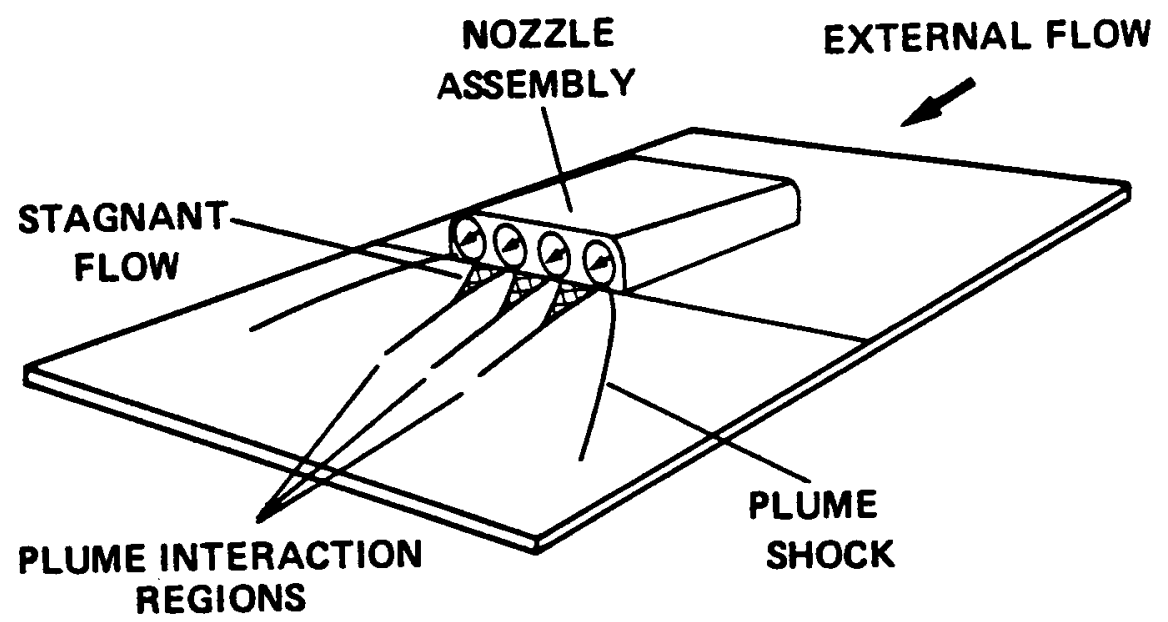

(a) overall view

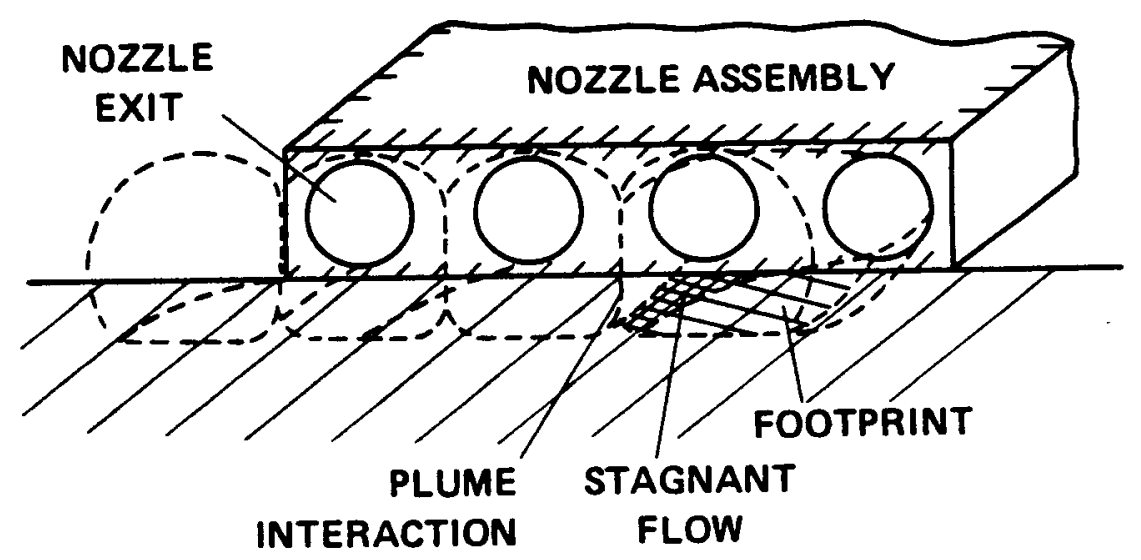

(b) detail near exit

Figure 8. Schematic of the interacting axisymmetric nozzle flow near plate. 


\section{ORIGINAL PAGE \\ COLOR PHOTOGRAPH}

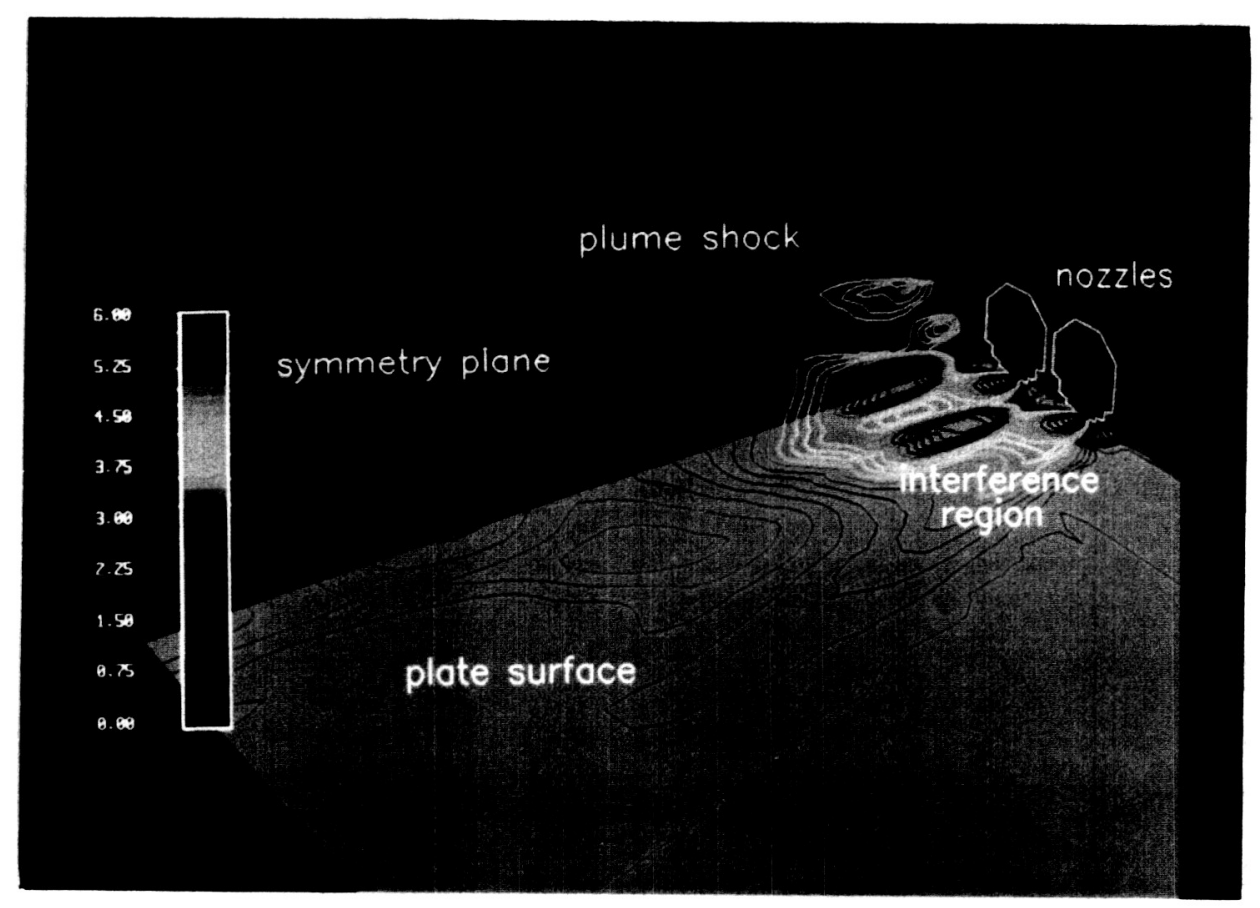

Figure 9. Perspective view of pressure contours on plate surface, at the symmetry plane, and at the inflow plane. $M_{\infty}=6, \alpha=5^{\circ}$, $\epsilon=0^{\circ}, \quad p_{e} / p_{\infty}=3$. 


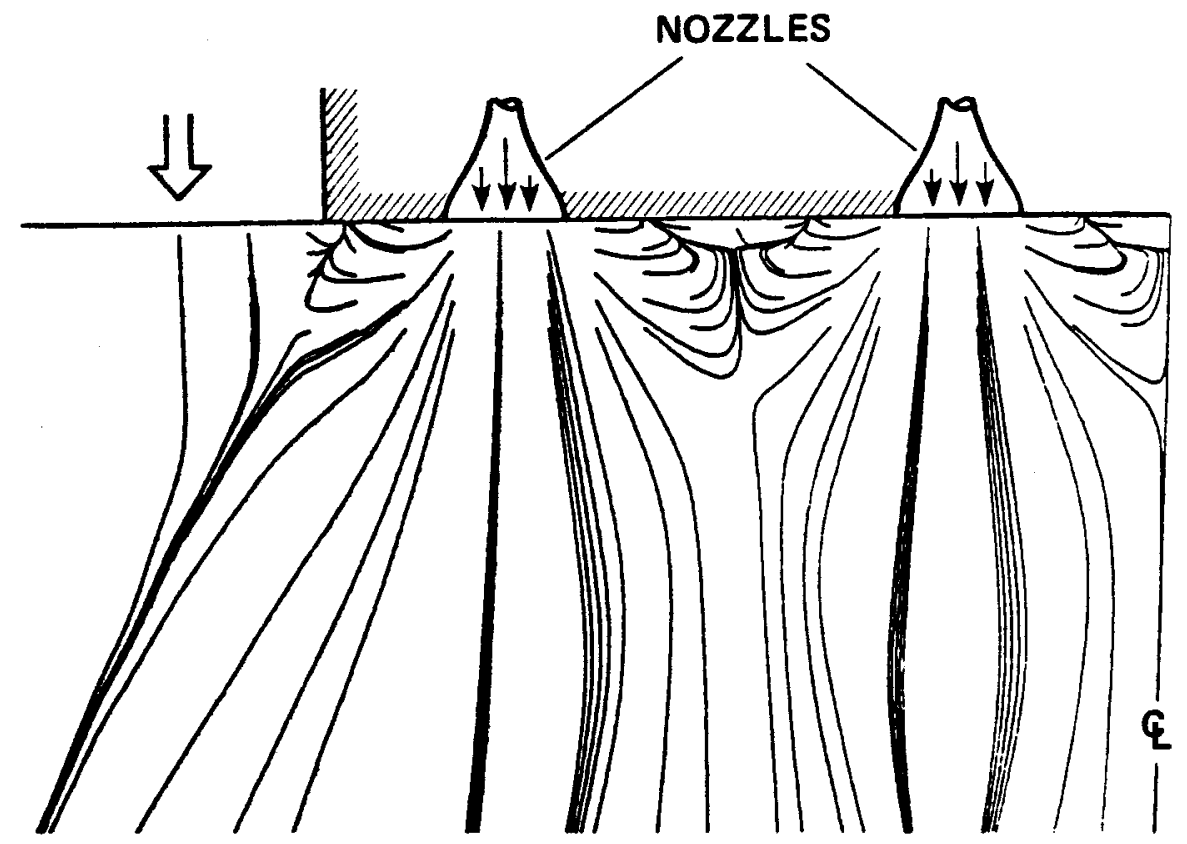

(a) calculated streamlines

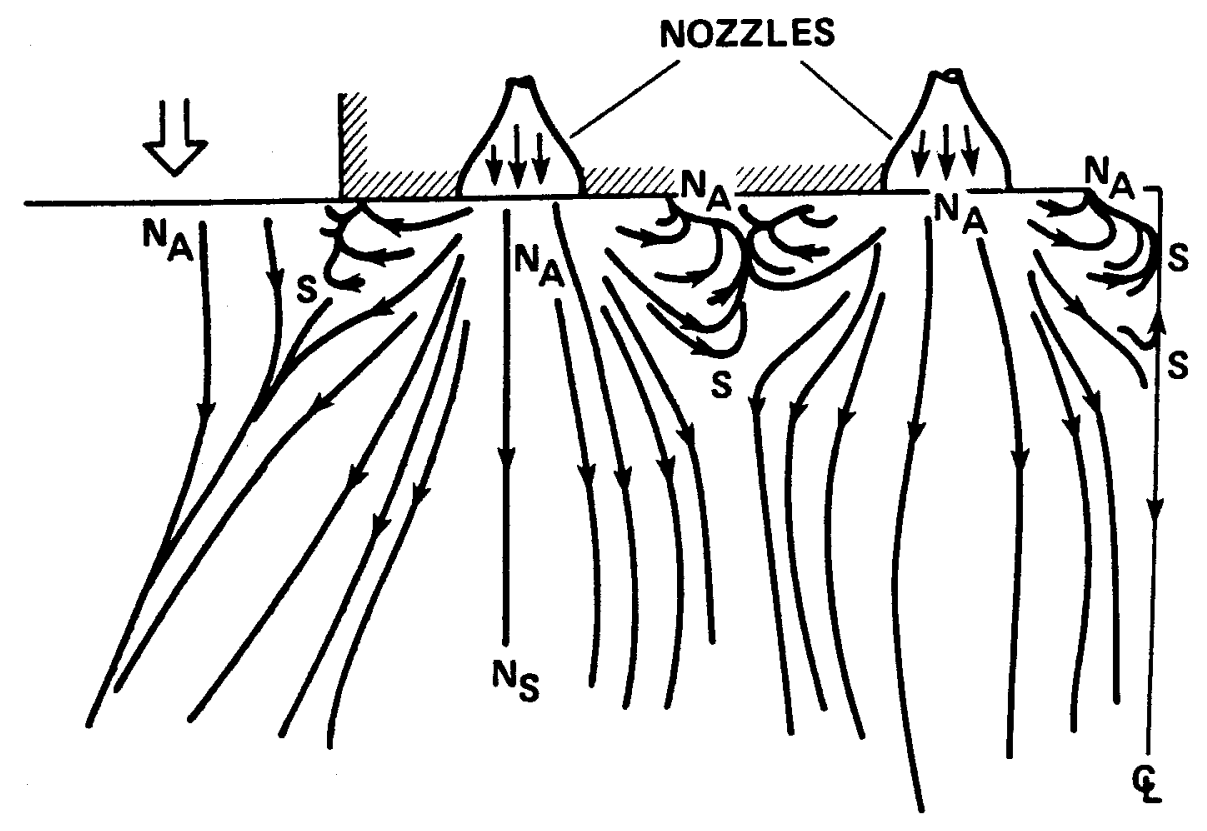

(b) postulated skin friction lines

Figure 10. Detail of surface flow pattern near the nozzle exit plane. $M_{\infty}=6$, $\alpha=5^{\circ}, \quad \epsilon=0^{\circ}, \quad p_{e} / p_{\infty}=3$. 


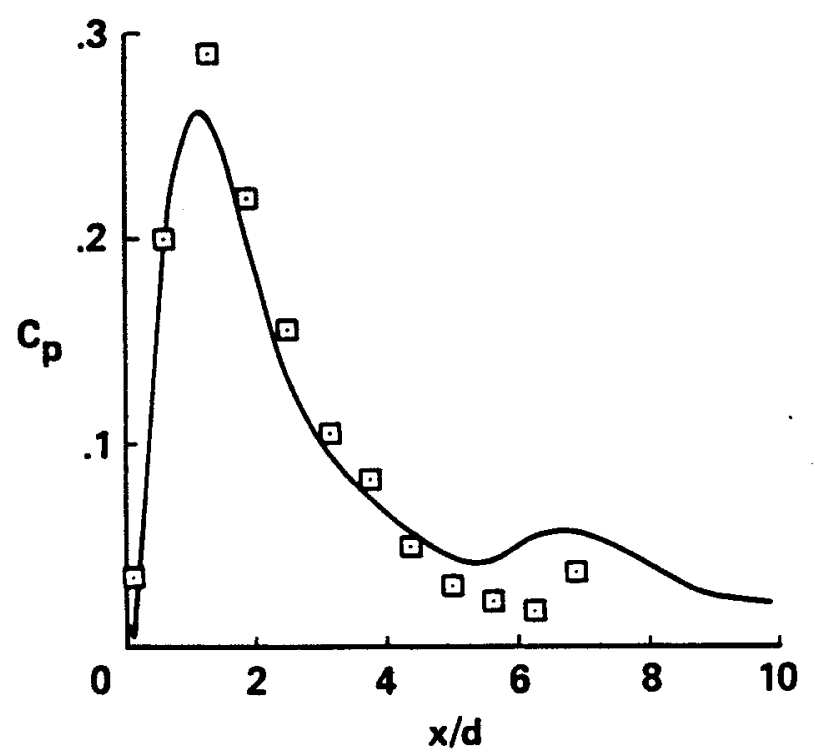

(a) between nozzles

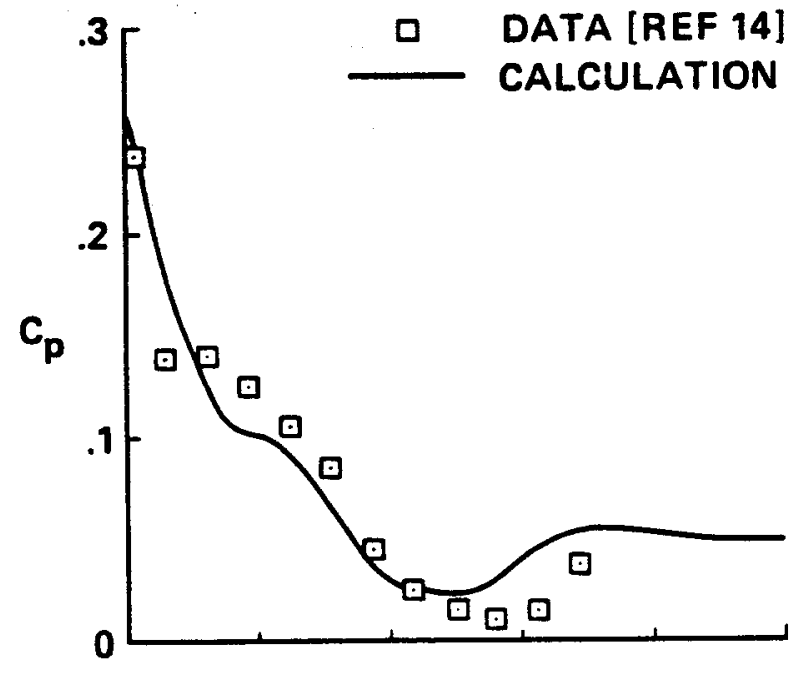

(b) nozzle centerline

Figure 11. Comparison of calculated and experimental surface pressure coefficients. $M_{\infty}=6, \quad \alpha=5^{\circ}, \quad \epsilon=0^{\circ}, \quad p_{e} / p_{\infty}=3$. 


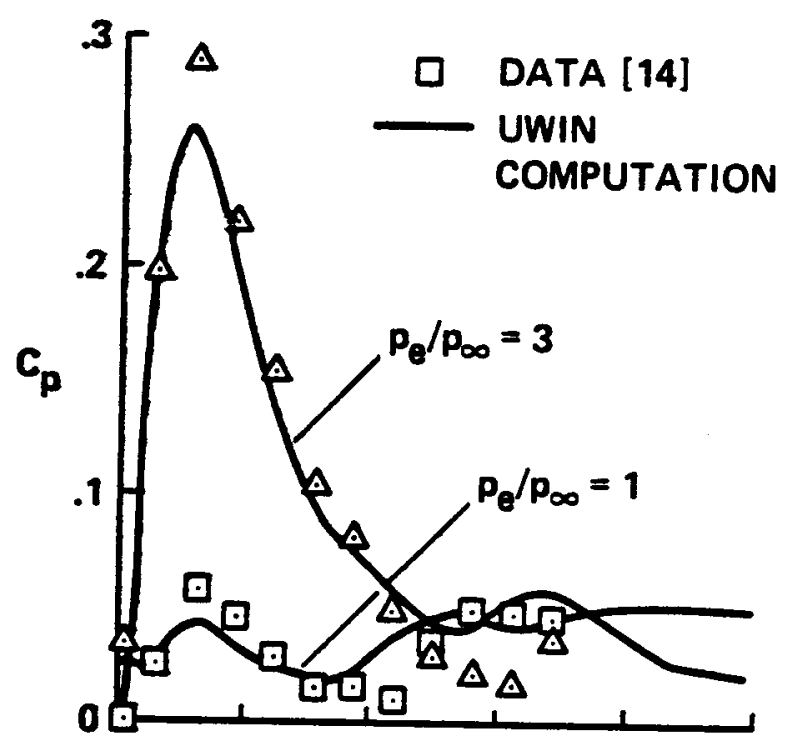

(a) between nozzles

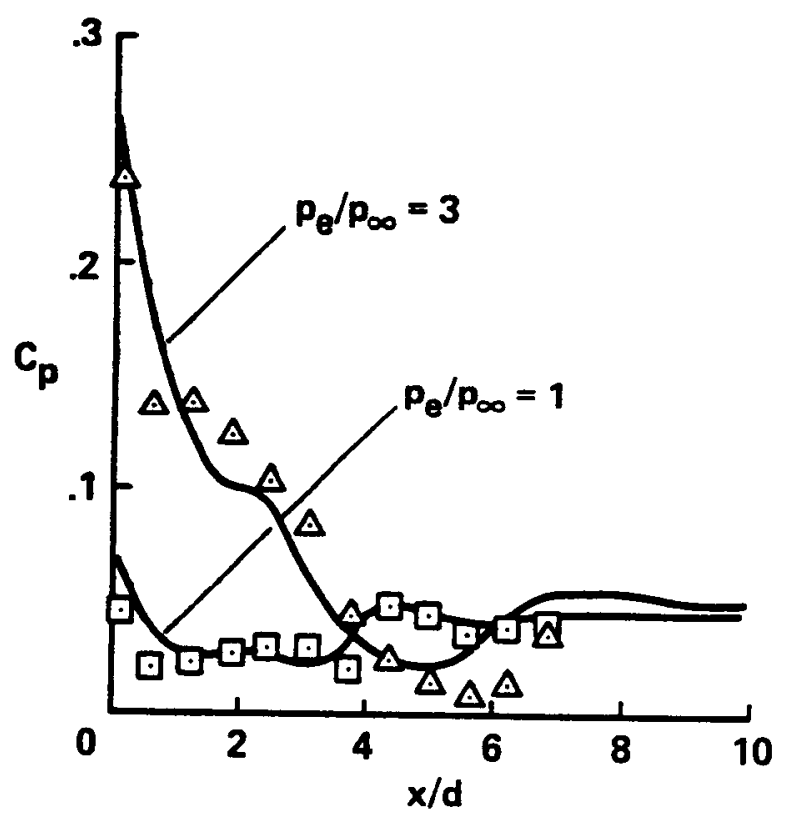

(b) nozzle centerline

Figure 12. Variation of surface pressure coefficient with exit pressure ratio. $M_{\infty}=6, \quad \alpha=0^{\circ}, \quad \epsilon=0^{\circ}$. 


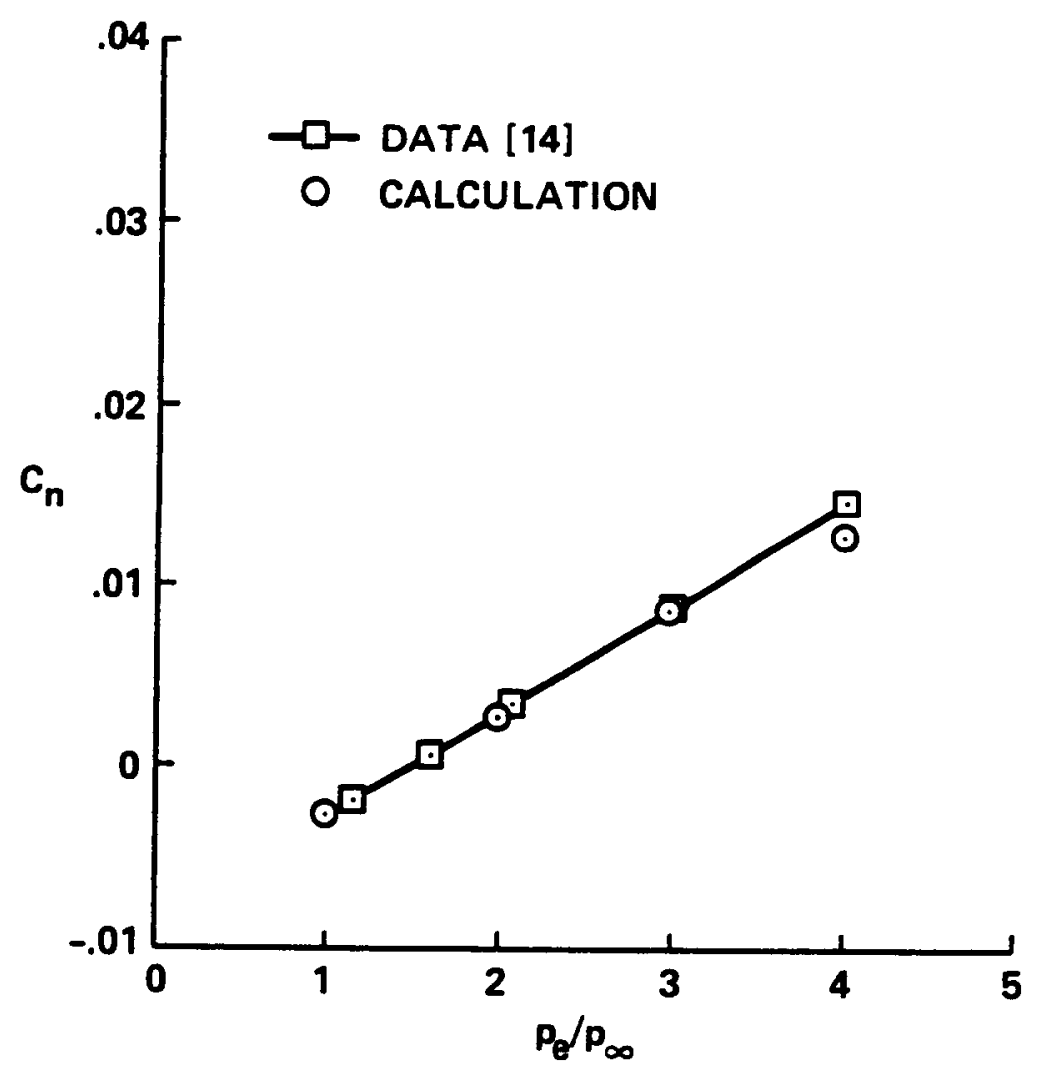

Figure 13. Variation of normal force coefficient with exit pressure ratio. $M_{\infty}=6, \alpha=0^{\circ}, \epsilon=0^{\circ}$. 


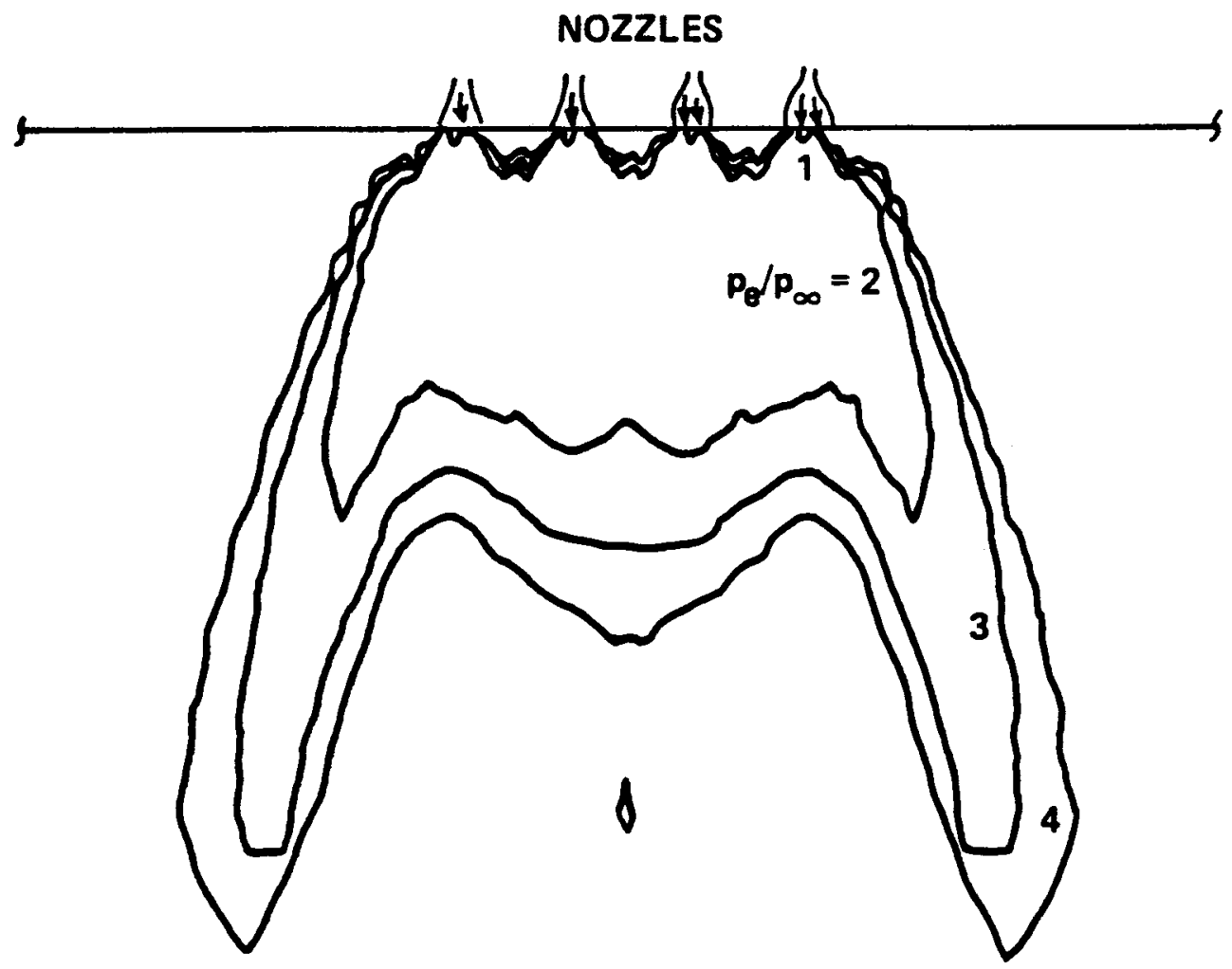

Figure 14. Comparison of footprint shapes for a progression of exit pressure ratios. $M_{\infty}=6, \alpha=0^{\circ}, \epsilon=0^{\circ}$. 


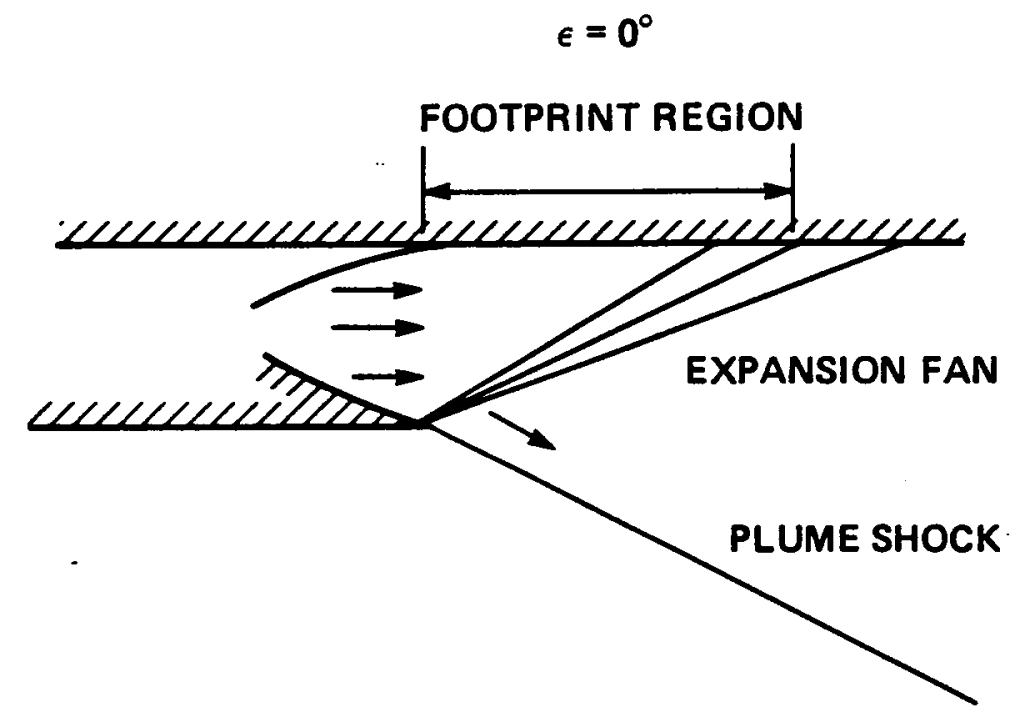

(a) without upsweep

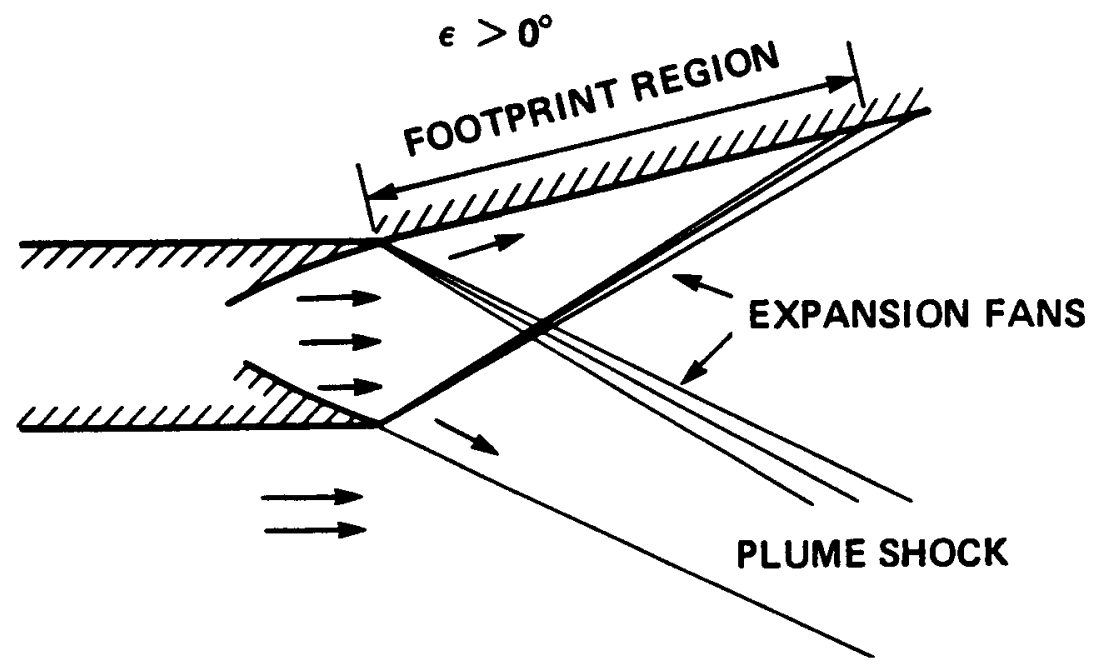

(b) with upsweep

Figure 15. Schematic comparison of an underexpanded nozzle flow field with and without upswept afterbody. 


\section{ORIGINAL PAGE \\ COLOR PHOTOGRAPH}

ORIGINAL PAGE

A... DUNTOGRAPH

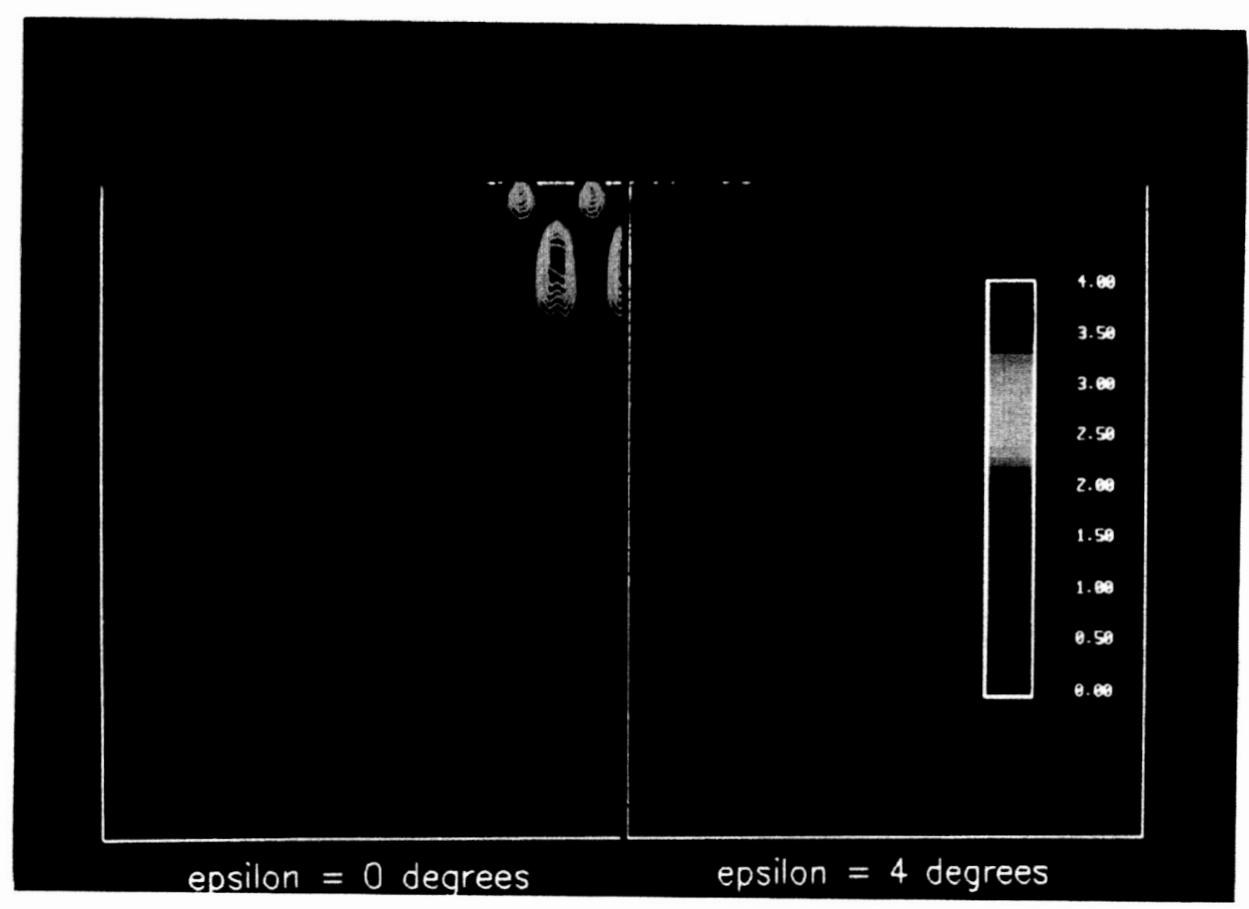

Figure 16. Comparison of surface pressure contours for flat and upswept afterbody profiles. $M_{\infty}=6, \quad \alpha=0^{\circ}, \quad p_{e} / p_{\infty}=3$. 


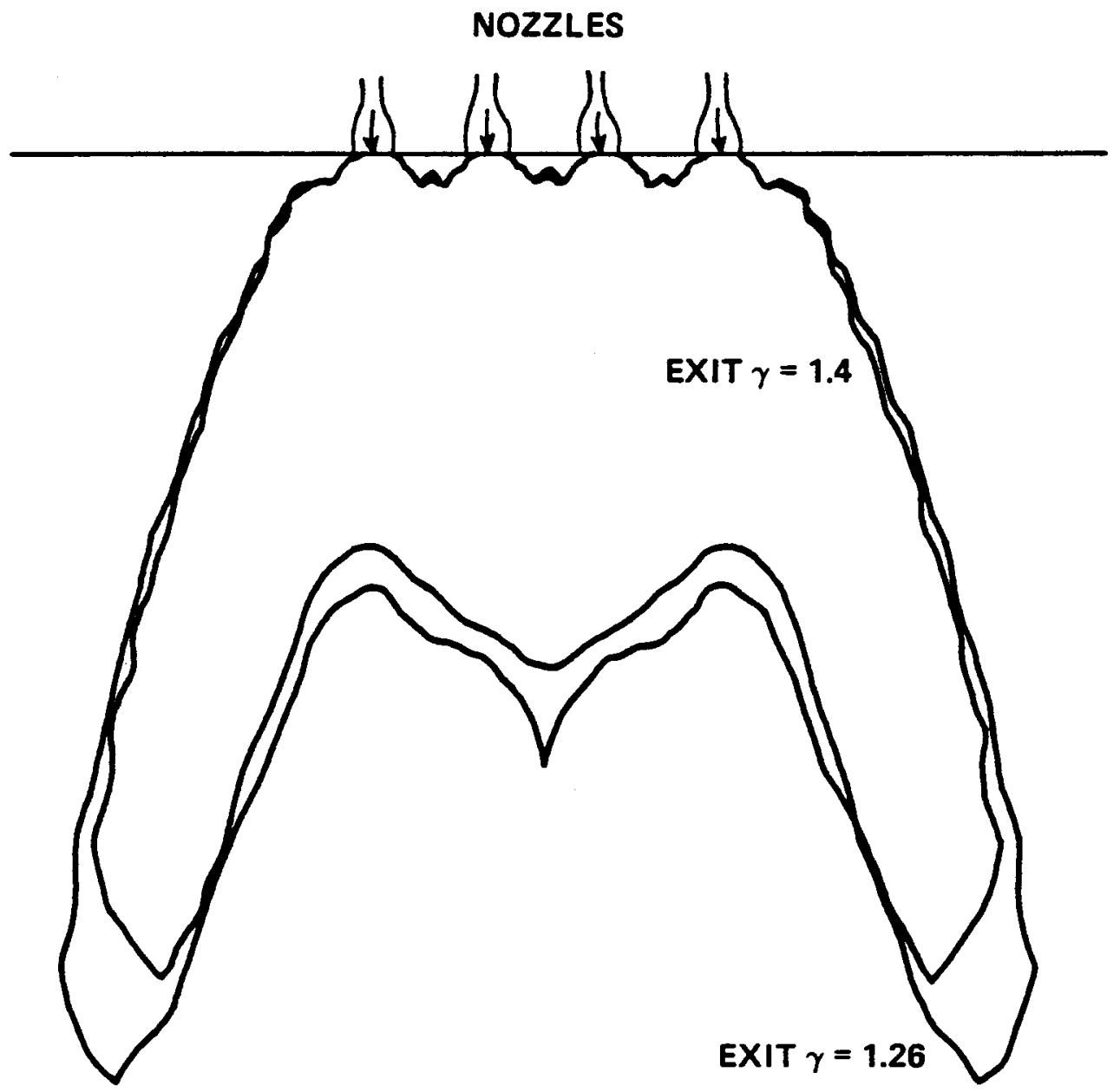

Figure 17. Comparison of footprint shapes for different exhaust gases. $M_{\infty}=6$, $\alpha=0^{\circ}, \epsilon=0^{\circ}, \quad p_{e} / p_{\infty}=4$. 


\section{ORIGINAL PAGE \\ COLOR PHOTOGRAPH}

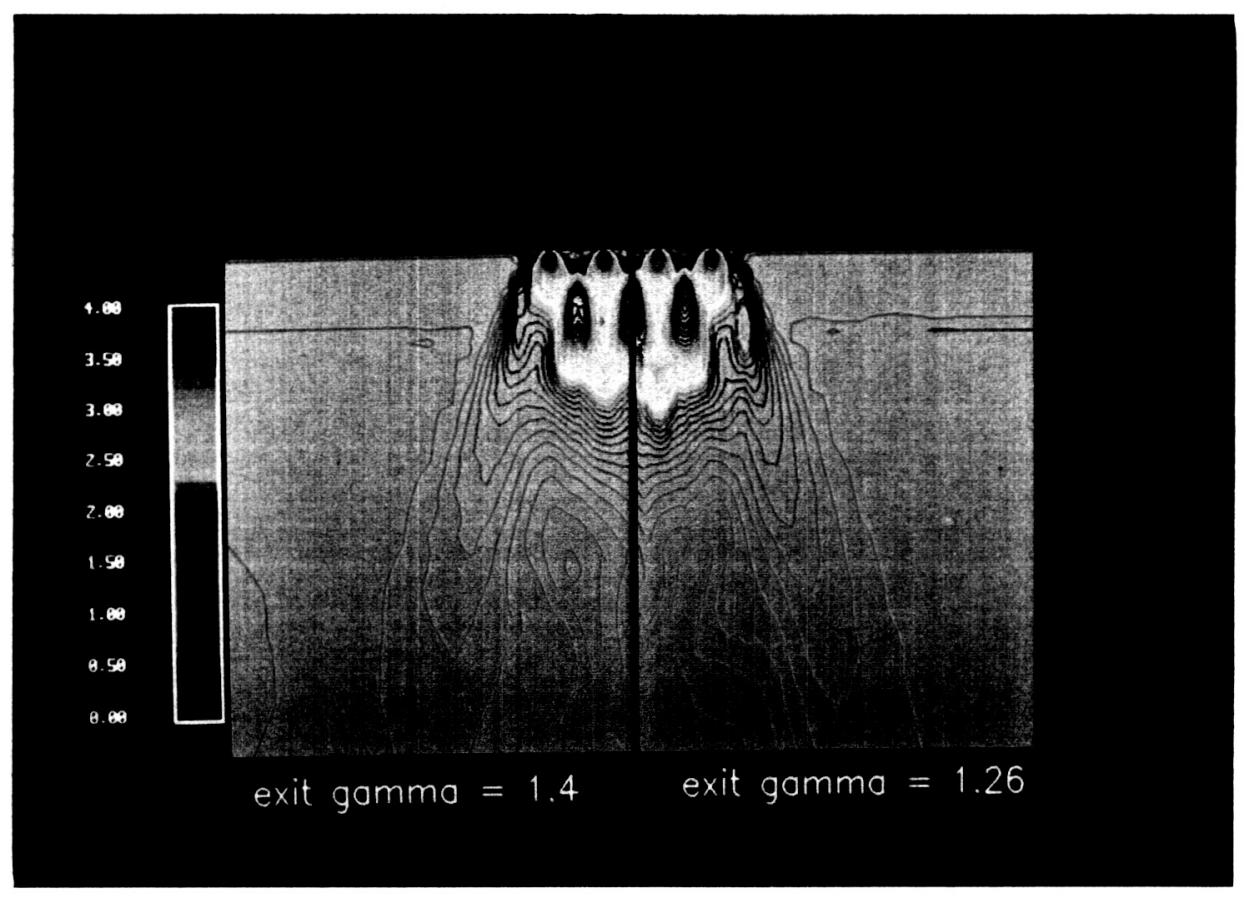

Figure 18. Comparison of surface pressure contours for different exhaust gases. $M_{\infty}=6, \quad \alpha=0^{\circ}, \quad \epsilon=0^{\circ}, \quad p_{e} / p_{\infty}=4$.

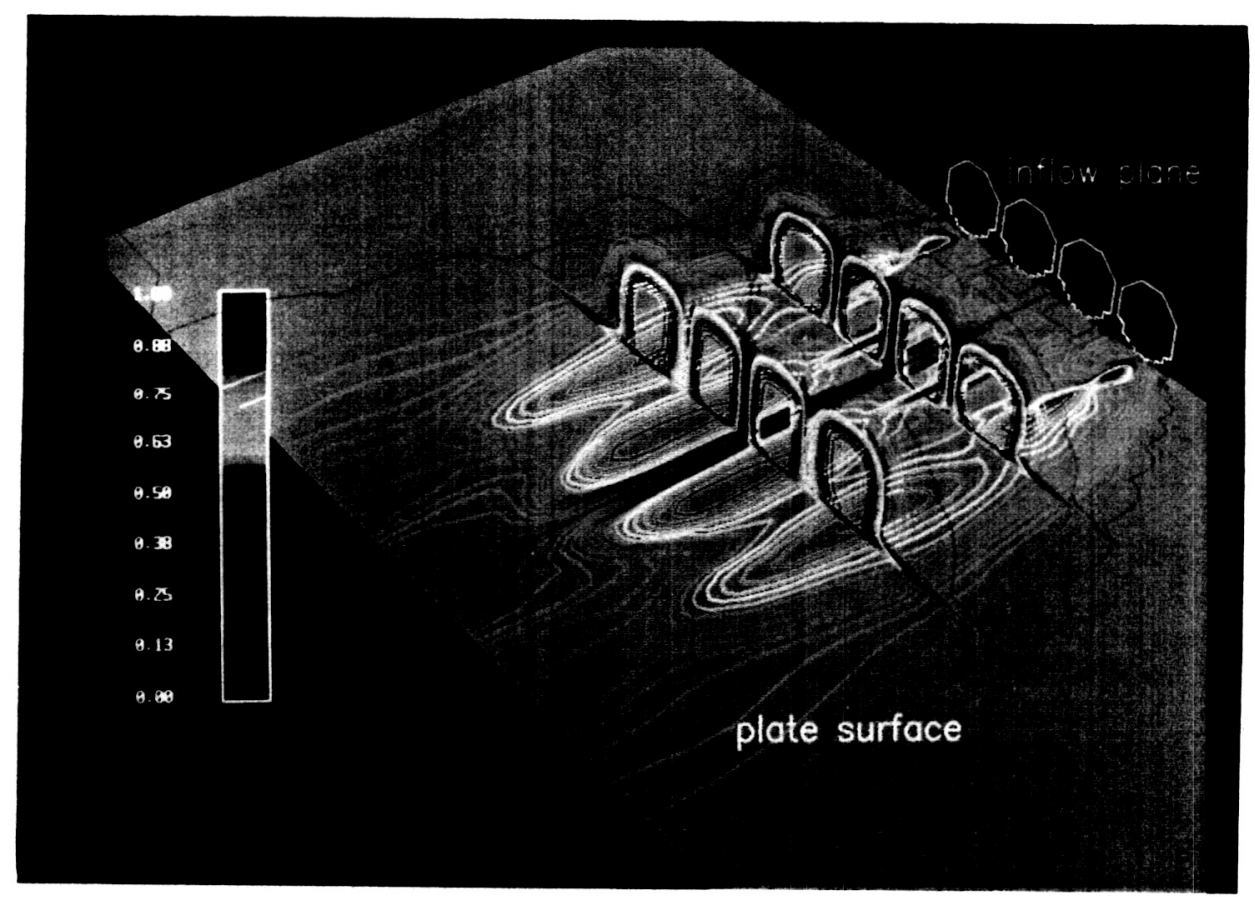

Figure 19. Perspective view of exhaust gas concentration contours. $M_{\infty}^{\vdots}=6$, $\alpha=0^{\circ}, \quad \epsilon=0^{\circ}, \quad p_{\mathrm{e}} / p_{\infty}=4, \quad \gamma_{\mathrm{e}}=1.26$. 


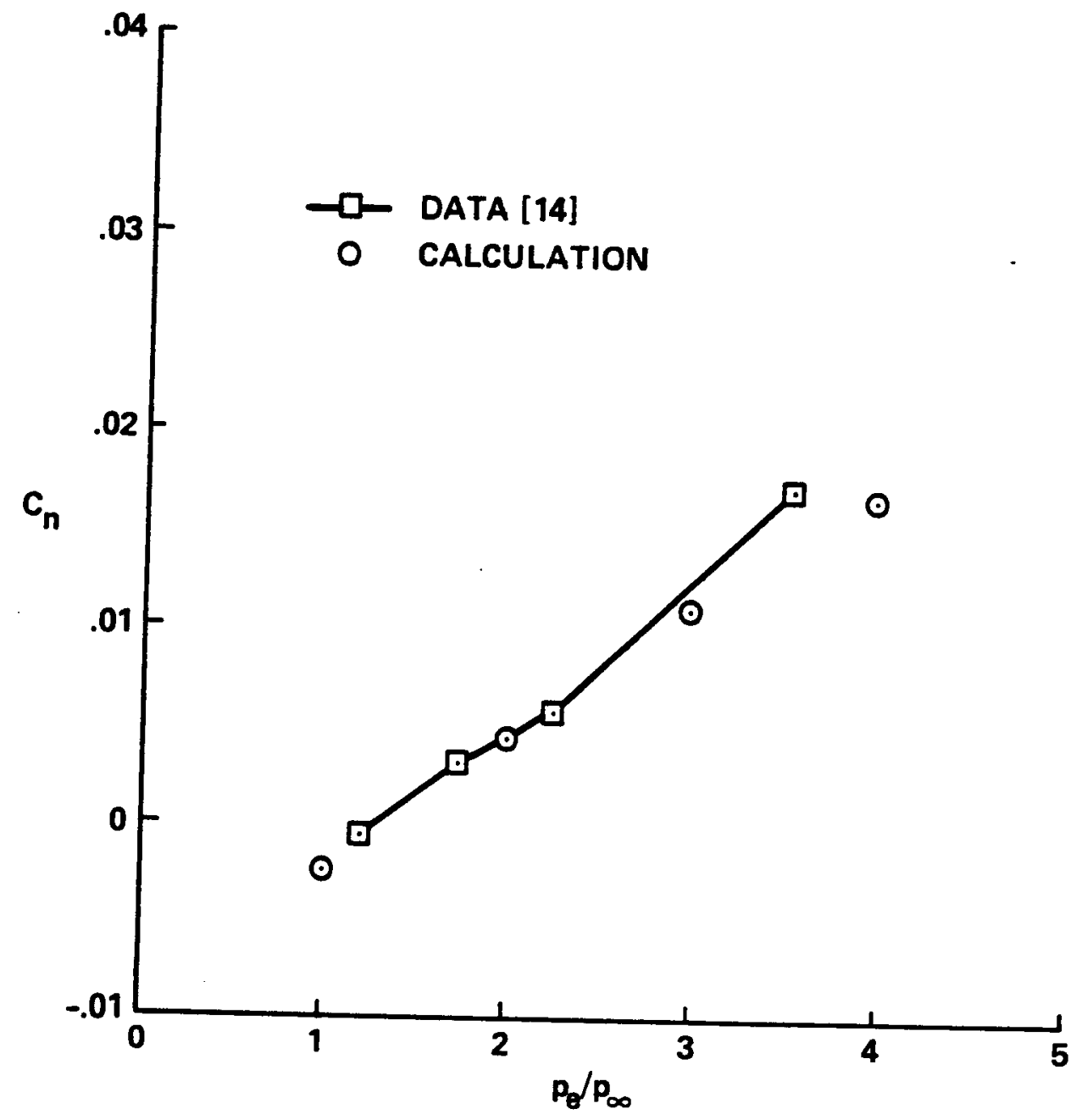

Figure 20. Variation of normal force coefficient with exit pressure ratio for $\mathbf{S F}_{6}$ exhaust gas. $M_{\infty}=6, \quad \alpha=0^{\circ}, \quad \epsilon=0^{\circ}$. 


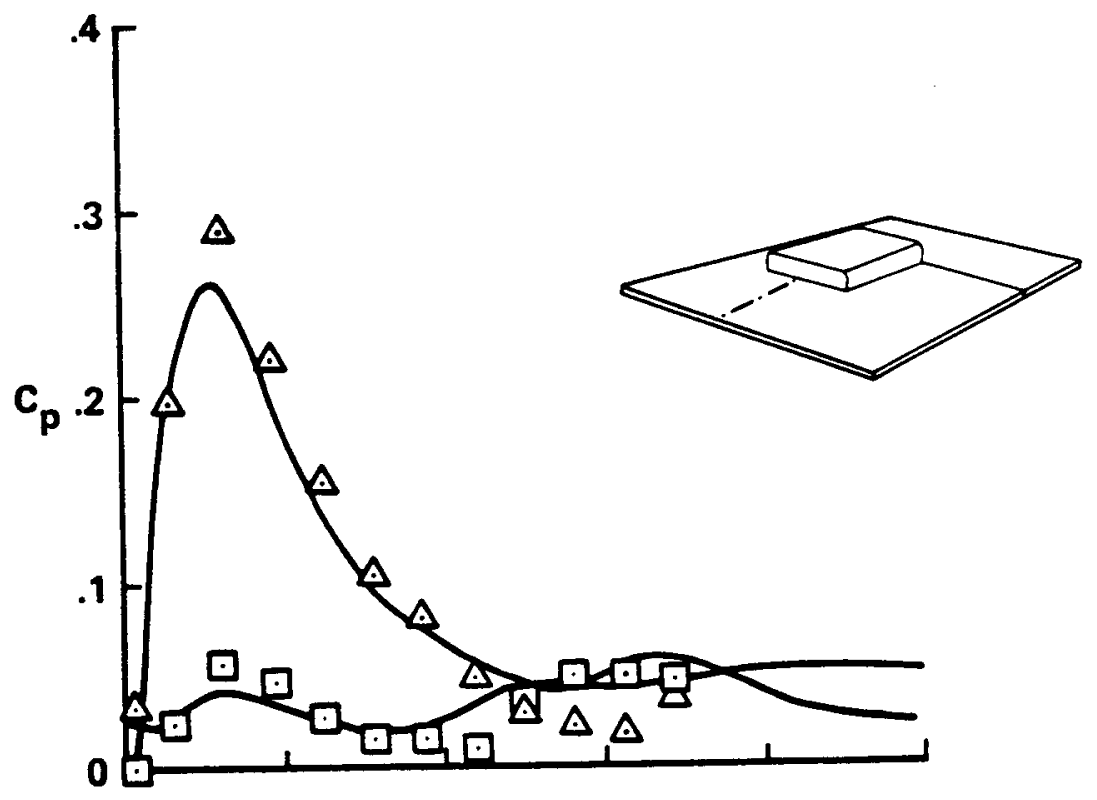

(a) axisymmetric nozzles

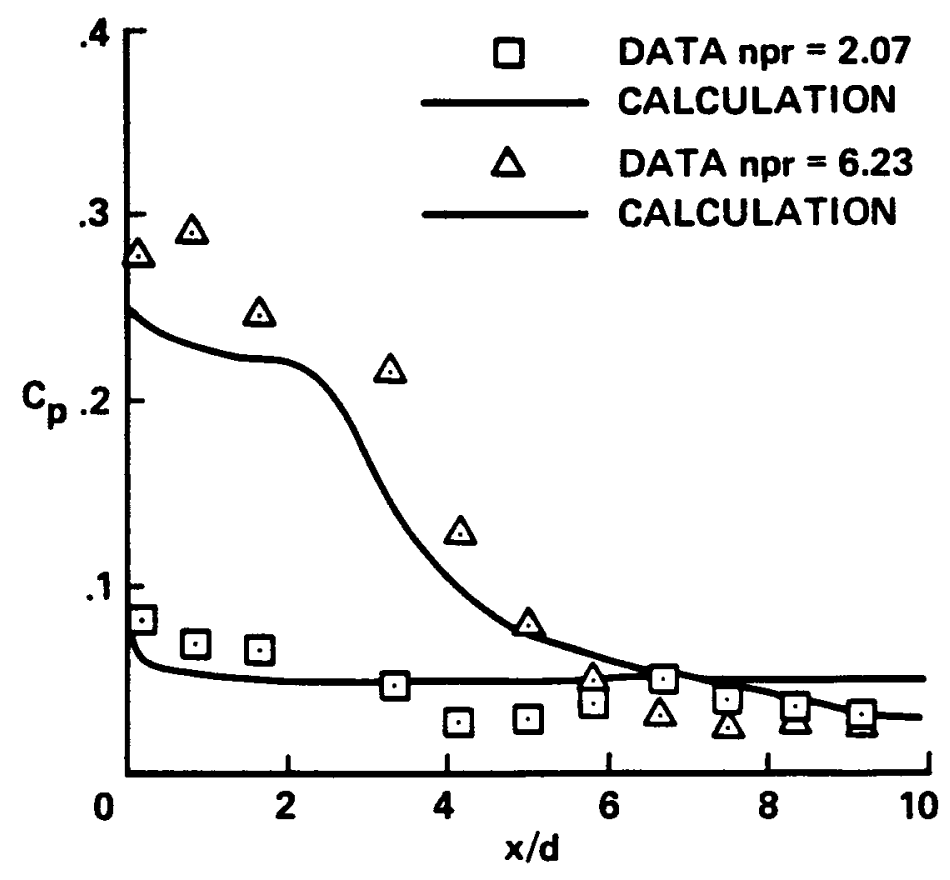

(b) slot nozzle

Figure 21. Comparison of calculated and experimental surface pressure coefficients for axisymmetric and slot nozzle geometries. $M_{\infty}=6$, $\alpha=5^{\circ}, \epsilon=0^{\circ}$. 


\section{ORIGINAL PAGE}

COLOR PHOTOGRAPH

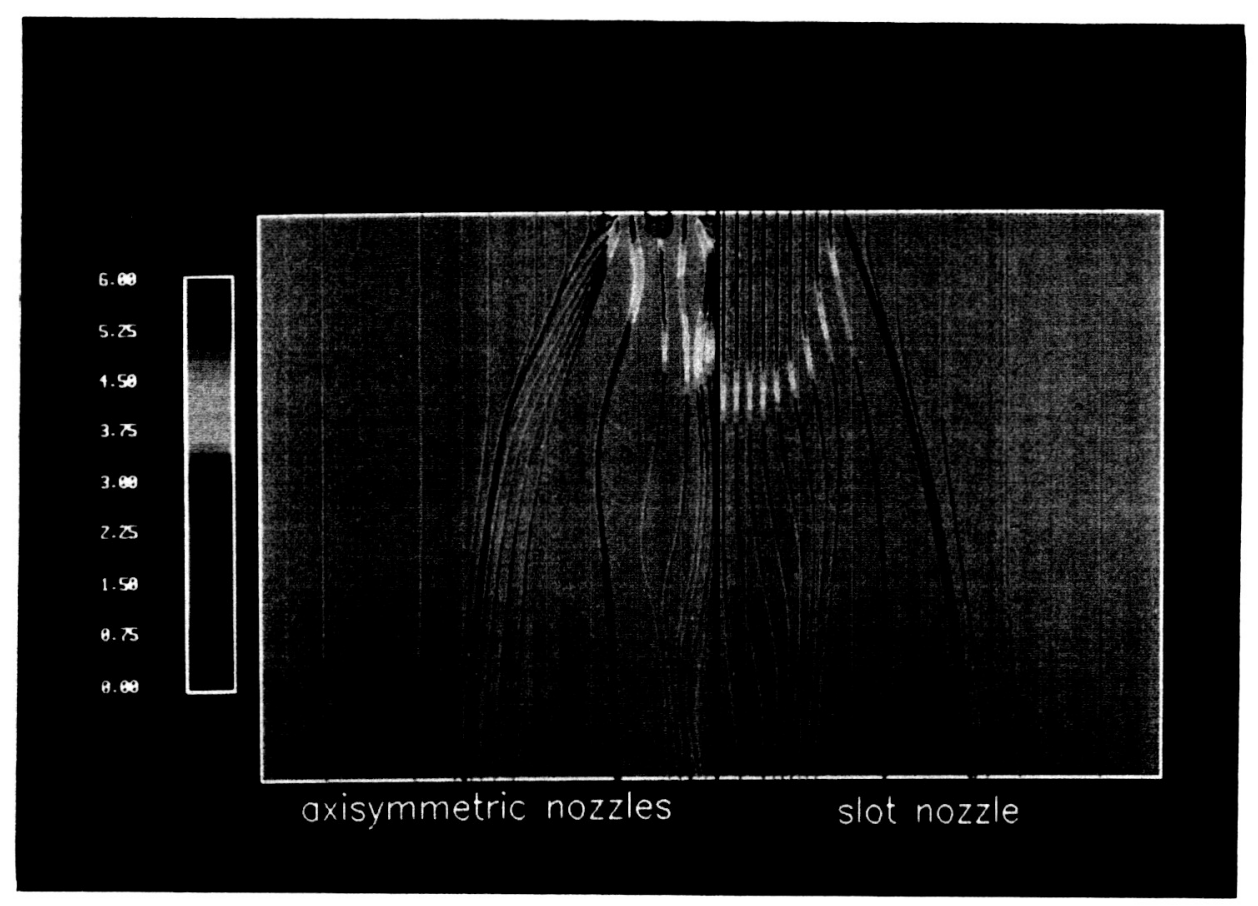

Figure 22. Comparison of limiting streamlines colored by pressure near nozzle exit for axisymmetric and slot nozzle geometries. $M_{\infty}=6$, $\alpha=5^{\circ}, \quad \epsilon=0^{\circ}, \quad p_{e} / p_{\infty}=6.23$. 

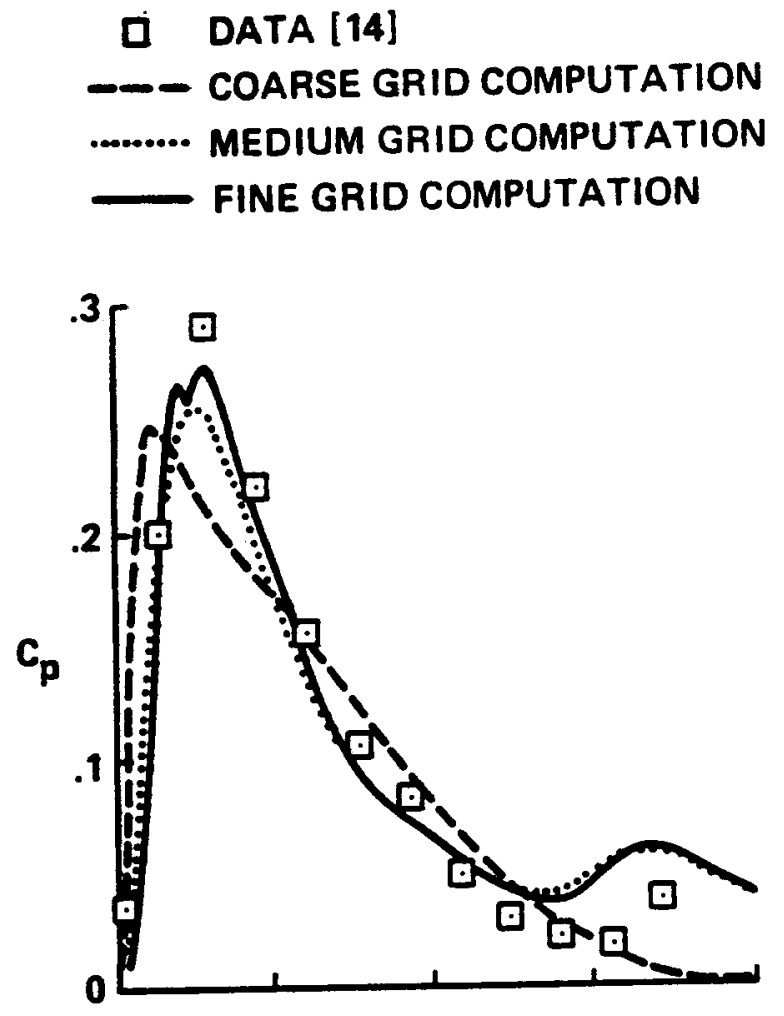

(a) between nozales

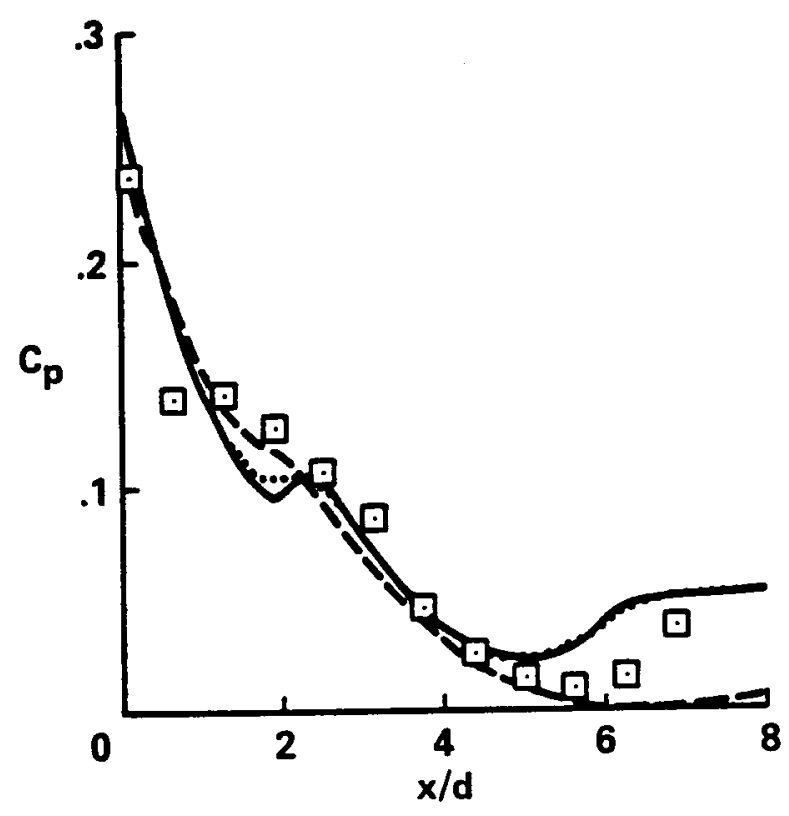

(b) nozzle centerline

Figure 23. Comparison of calculated and experimental surface pressure coefficients for grid refinement study. $M_{\infty}=6, \alpha=5^{\circ}, \epsilon=0^{\circ}$, $p_{e} / p_{\infty}=6.23$. 


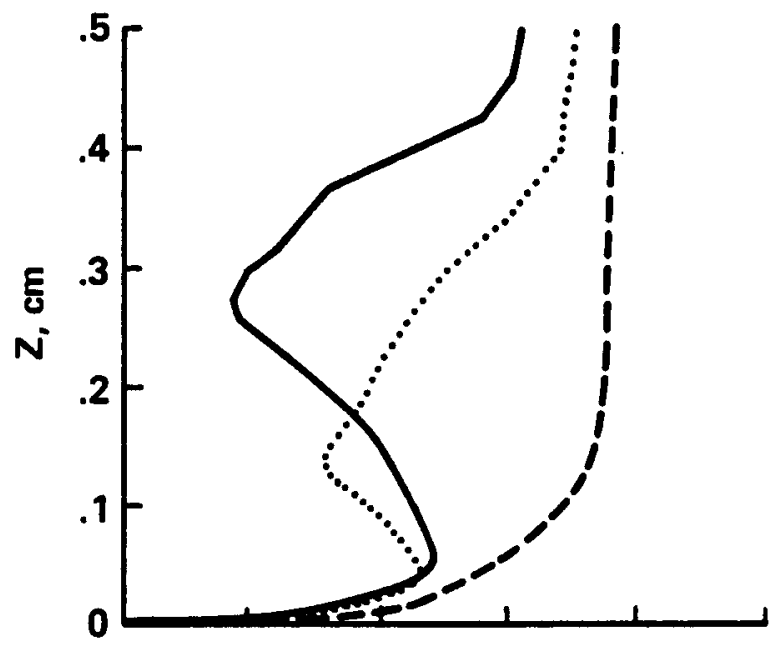

(a) between nozzles

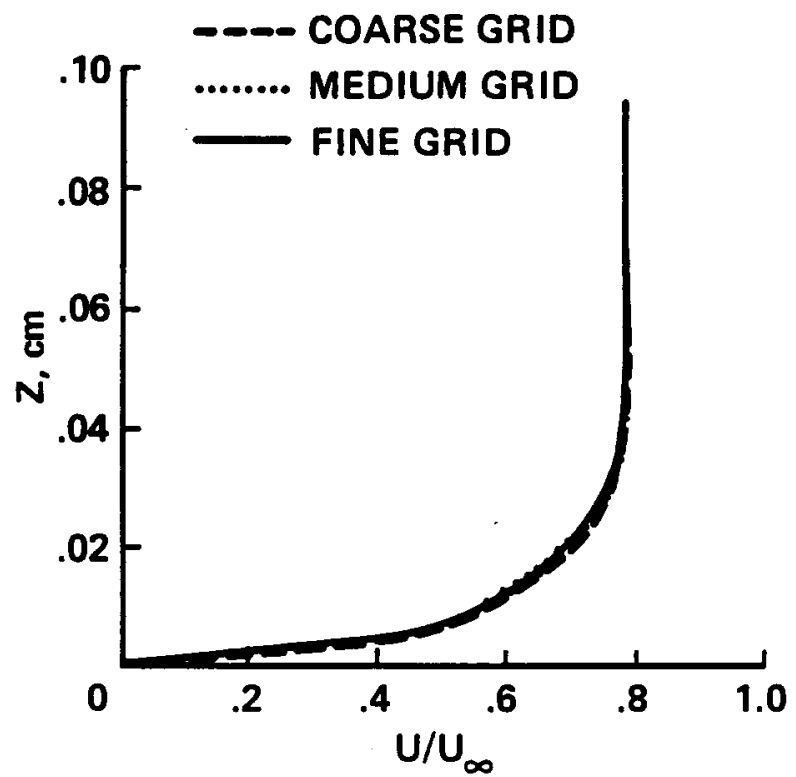

(b) nozzle centerline

Figure 24. Comparison of boundary layer profiles at $x / d=1$ for grid refinement study. $M_{\infty}=6, \alpha=5^{\circ}, \epsilon=0^{\circ}, \quad p_{e} / p_{\infty}=6.23$. 


\section{CHAPTER FOUR}

\section{ANALYSIS OF GENERIC HYPERSONIC VEHICLE FLOW FIELDS}

\subsection{Introduction}

In the preceding chapter, it was shown that propulsion system design parameters have a large influence on the afterbody flow field and vehicle forces. It is now appropriate to address questions relating to installed scramjet performance: how does the impinging exhaust plume affect vehicle balance? Can proposed nozzle exit conditions produce enough thrust to overcome drag? How does the exhaust plume interact with a nonuniform external flow? What impact does thrust vectoring have on vehicle forces and moments? These are some of the questions that will be addressed in this chapter. Investigating these issues required a realistic hypersonic vehicle geometry, including an exhaust nozzle, with experimental data available for a power-on flight condition. Though experimental activity in this area is in progress (see reference [20], for example), little data already exists. The fact that most candidate experiments are classified exacerbated the difficulty in locating data. The generic hypersonic vehicle from Lockheed-Georgia (private communication with D. Schuster, Lockheed-Georgia Co., Marietta, Georgia)) best filled these requirements.

The generic hypersonic vehicle geometry provided a unique opportunity to solve power-on flight conditions for a realistic configuration, and to compare those results with experimental data. This chapter presents the results of power-on solutions for this body at supersonic and hypersonic speeds. The experiment included tests at transonic and supersonic conditions; experimental apparatus and procedures for these tests are described first. A discussion of formulating the numerical problem includes surface geometry definition, grid generation, and boundary conditions. Three-dimensional grid generation by means of a two-dimensional grid generator is summarized. Also, the boundary conditions and solution procedure presented in the previous chapter are modified to accomodate this geometry as explained below.

Flow field analyses for the generic hypersonic vehicle are presented in two segments. Supersonic $\left(M_{\infty}=1.46\right)$ solutions at a series of exit pressure ratios are compared with experimental data. The afterbody flow field is described, and the effect of the exhaust plume on vehicle forces and moments is enumerated. Next, solutions are presented at a practical hypersonic cruise condition $\left(M_{\infty}=6\right)$. These results demonstrate that the flow field of this strongly interacting exhaust plume is rich in information about afterbody aerodynamics. The chapter concludes with a study of thrust vectoring as a possible means to balance the vehicle.

\subsection{Experimental Apparatus and Procedure}

The generic hypersonic vehicle model was designed and tested by Lockheed-Georgia to study the transonic and supersonic performance of a NASP-like configuration. The model, pictured in figure 25, features a pointed nose, blended wing-body, and exhaust nozzle with afterbody contouring. The overall vehicle length is $50 \mathrm{~cm}$, and the wing semi-span reaches a maximum of $10 \mathrm{~cm}$. In order to calculate force 
and moment coeficients for the numerical solutions, a reference area and reference length were required. The approximate planform area $\left(500 \mathrm{~cm}^{2}\right)$ and body length $(50 \mathrm{~cm})$ provided these values. The afterbody upsweep at the centerline is 20 degrees, and the nozzle is $0.5 \mathrm{~cm}$ high. Power-on simulations were accomplished by blowing air through the nozzle. The model was supported by a blade mount attached to the upper surface; the wing shielded disturbances created by the mount so that it would not interfere with the nozzle flow. High pressure lines for the nozzle effluent were ducted through the mount to an internal plenum. Pressure taps were placed on the model lower surface downstream of the nozzle, on the centerline and at a few off-centerline locations.

The tests were performed in the Lockheed 20 by 28 -inch compressible flow tunnel at freestream Mach numbers from 0.8 to 1.46. At $M_{\infty}=1.46$, tests were conducted for nozzle exit pressure ratios from 0 (power off) to 6.27. The tests employed a "cold" jet, meaning that its stagnation temperature was equal to the freestream stagnation temperature. By design, the exhaust flow was to be choked at the exit plane, but manufacturing imperfections caused the flow to choke inside the model. Exit plane measurements showed that the exit Mach number varied circumferentially, and ranged approximately from 1.12 to 1.2. The tunnel stagnation pressure was $1.4 \mathrm{~atm}$, stagnation temperature was $396 \mathrm{~K}$, and the Reynolds number was 230,000 $\mathrm{cm}^{-1}$.

\subsection{Numerical Problem Formulation}

4.3.1 Geometry definition and grid generation- The geometry definition for this model was supplied in the form of body-axis-normal cross section coordinates at specified stations along the length of the body. To produce a smooth grid with adequate resolution, it was necessary to generate new cross sections by interpolating between succesive body stations. Not only did this yield a smoother distribution of grid points in the streamwise direction, but it also improved resolution of the afterbody downstream of the nozzle, where rapid plume expansion occurs.

The three-dimensional grid was generated using a two-dimensional hyperbolic grid generation procedure [53] at each cross section. This technique solves a system of hyperbolic equations for the grid coordinates $(y, z)$ in the cross sectional plane. Because the system of equations is hyperbolic, the solution procedure marches from the body outward to the outer boundary. Consequently, generating grids is computationally efficient (approximately $50 \mu \mathrm{sec}$ of Cray XMP CPU time per grid point for these grids), and improvements or alterations to the grid are accomplished quickly.

The two-dimensional grids were then assembled to form a three-dimensional grid about the entire body, producing a cylindrical, or O-H, grid topology as shown in figure 26. This method and grid topology are quite amenable to blended bodies. At the nozzle exit plane, where the geometry has two cross sections, grids were generated for the section both including and excluding the nozzle contour, as shown in figure 27. This resulted in a planar zonal interface that was not one-to-one. The UWIN code features a trilinear interpolation routine wherein coefficients are computed at each interface grid point. Since the grids are stationary, these coefficients are computed only once, and are subsequently used to transfer information across the interface.

The grid was then segmented into four sequential zones, each of which had 16 points in the stream direction, 89 points in the circumferential direction, and 50 points in the radial direction. Disregarding 
the overlap planes, the computational domain consisted of 244,750 grid points. Exponential clustering in the radial direction resulted in a normal spacing of the first point off the body of about $0.01 \mathrm{~cm}$. At $M_{\infty}=1.46$, this spacing equated to $y^{+} \approx 5$, while at $M_{\infty}=6, y^{+}$was about 1 .

4.3.2 Boundary Conditions- For bodies with sharp or blunt noses in supersonic flow, boundary condition procedures customarily require that the upstream boundary be placed sufficiently far from the body to capture the bow shock. However, the formulation of the Osher scheme in the UWIN code possesses a known problem (private communication with J. Flores, NASA Ames Research Center, Moffett Field, California) in solving the flow along the singular grid line upstream of the body. Work on this problem is in progress, but in the meantime an alternative is needed. One possibility is to generate a starting solution with another code, and use that as fixed inflow data to the UWIN code at a body station downstream of the nose. This method is cumbersome, though, and since codes make different approximations and errors, information transfer between codes introduces errors at the inflow plane.

A viable alternative for this study was devised by placing the upstream boundary at the pointed nose of the body. Thus, the inflow plane was a true polar grid, with the only point on the body being at the origin. Flow conditions on this plane were fixed to be freestream at every point except the origin, where flow conditions were specified based on tabulated normal shock conditions [52]. These conditions make sense from a physical standpoint because in supersonic flow, disturbances propagate only downstream; hence at the nose, the only point where the flow "knows" about the body is the origin. Evaluation of the results indicates that this boundary condition did indeed produce a realistic inflow condition.

The other boundary conditions were specified as before: symmetry conditions at the windward and leeward pitch planes of symmetry, freestream at the outer boundary, no slip and $\partial p / \partial n=0, \partial T / \partial n=0$ at the body, and supersonic outflow at the downstream boundary. At the nozzle exit plane, flow variables at points within the nozzle circumference were specified from the exhaust conditions, while variables at points outside the nozzle were specified by interpolation from the interface plane of the upstream zone.

\subsection{Solution Procedure}

While supersonic flows over streamlined bodies such as this can be solved efficiently by parabolized Navier-Stokes methods [31], these codes cannot solve flows with streamwise separation. As will be shown shortly, interactions between the exhaust plume and the external flow do precipitate streamwise separation in some instances. Also, part of the intent of this work was to demonstrate a single code, tip-to-tail solution capability. As such, the UWIN code solved the entire flow field around the generic hypersonic vehicle. First, a power-off solution was generated by imposing no-slip conditions on the face of the exhaust nozzle. Transition was assumed at the nose, with turbulent flow prevailing over the entire body surface. Subsequent power-on solutions were obtained by specifying nozzle exhaust conditions, and iterating the two downstream zones. Iterating the two upstream zones was unnecessary because in these flow fields, upstream influence of the nozzle is localized. The effect of the exhaust plume on the upstream flow was easily contained within the zone immediately upstream of the nozzle.

Solutions were run on the Cray X-MP, and the initial power-off solutions converged in about 400 iterations, requiring about 4 hours of CPU time. Subsequent power-on solutions required about 500 iterations of just the two downstream zones, taking about $2.8 \mathrm{CPU}$ hours. 


\subsection{Generic Hypersonic Vehicle Flow Solutions at $M_{\infty}=1.46$}

In order to correlate the numerical results with available experimental data, the generic hypersonic vehicle geometry was first studied at a freestream Mach number of 1.46 and an angle of attack, $\alpha$, of zero degrees. A series of exit pressure ratios were tested, from $p_{e} / p_{\infty}=0$ (power off) to $p_{e} / p_{\infty}=6.27$. This section presents a description of the flow fields, comparisons to experimental data, and determination of vehicle forces. To begin, pressure contours for the entire solution domain are shown in figure 28 at $p_{e} / p_{\infty}=6.27$. Many interesting phenomena apparent in this flow field merit further study: formation of shocks at the bow and at the wing, and their eventual interaction; heat transfer peaks at the nose and sharp leading edge of the wing; and the flow pattern around the square wing tip, to name a few. Unfortunately, these aspects are beyond the scope of this investigation, and will be set aside. Instead, the windward-side flow field on the afterbody is of current interest, and the details of this region are now explored.

Figure 29 shows a detailed view of the exhaust plume pressure contours on the body and in the symmetry plane. The plume shock is readily evident, as is the footprint of the exhaust flow on the afterbody. Because the exhaust flow is at a relatively low Mach number $\left(M_{e} \approx 1.2\right)$, expansion waves propagate into the plume at a large angle to the local flow direction, and expansion occurs a relatively short distance downstream of the nozzle. An expansion fan is seen behind the plume shock, a result of the nozzle flow expanding along the upswept afterbody. Near the end of the body, a recompression brings the overexpanded flow back up to ambient pressure.

Figure 30 presents surface pressure coefficient comparisons on the windward symmetry plane downstream of the nozzle exit for a series of exit pressure ratios. The data show fairly good correspondence for all values of $p_{e} / p_{\infty}$. Expansion to ambient pressure takes place within about $2.5 \mathrm{~cm}(\approx 5$ nozzle heights). The calculated pressure is seen consistently to overpredict the data in the initial expansion region of the exhaust plume. This is due, at least in part, to nonuniform experimental exit plane conditions wherein the exit Mach number (and hence, the pressure) varied circumferentially across the nozzle. Exit plane measurements show that the exit plane pressure was highest at the symmetry plane. Because the calculations assumed uniform exit plane conditions, the flow did not expand as rapidly at the symmetry plane as in the experiment. From these comparisons, it is expected that the calculated normal force on the afterbody will be slightly higher than what would have been obtained experimentally; a corresponding increment to lift and thrust will result.

With that in mind, lift and thrust are plotted as a function of exit pressure ratio in figure 31 . Because this model has no inlet, the forces at the nozzle face were adjusted by subtracting the freestream momentum flux and pressure. This renders a somewhat realistic representation of the actual forces on the entire vehicle, by assuming that flow conditions at the inlet are freestream. The variation of lift and thrust with exit pressure is linear, commensurate with the results of section 3.6.1 for the nozzle/afterbody model. The figure indicates that net thrust is first generated at $p_{e} / p_{\infty} \approx 2.25$. Table 3 lists the incremental contributions to lift and thrust from the nozzle and afterbody at $p_{e} / p_{\infty}=6.27$. Incremental values represent the difference between the power-on quantity and the power-off quantity. The table indicates that the thrust contribution from the afterbody is negligible compared to the contribution from the nozzle face. However, a significant lift increment is generated by the afterbody. 
Table 3. Incremental lift and thrust due to nozzle and afterbody.

$$
M_{\infty}=1.46, \quad \alpha=0^{\circ}, \quad p_{e} / p_{\infty}=6.27 \text {. }
$$

\begin{tabular}{|l|c|c|}
\hline Component & $c_{T}-c_{T_{0}}$ & $c_{L}-c_{L_{0}}$ \\
\hline Nozzle & 0.1154 & 0. \\
\hline Afterbody & 0.0049 & 0.0151 \\
\hline Vehicle & 0.1206 & 0.0160 \\
\hline
\end{tabular}

Nozzle and afterbody forces are also important to vehicle balance. As forces on the nozzle face and afterbody increase, vehicle balance changes. The pitch-axis moment coefficient, $c_{m_{y}}$, provides a convenient way to quantify vehicle balance. Since the vehicle weight and center of gravity were not provided, moments were calculated about the nose of the body. Figure 32 shows the variation of $c_{m}$, with exit pressure. The pitch moment decreases with increasing pressure, indicating that the nose-down moment due to additional afterbody lift overcompensates for the nose-up moment due to additional thrust forces at the exit face as the exit pressure increases. Because afterbody lift acts at a large distance from the center of gravity, its effect on overall vehicle balance is considerable. Including the moment due to vehicle weight would, of course, increase the nominal value of $c_{m}$, substantially.

Limiting streamlines on the afterbody are presented in figure 33 at $p_{e} / p_{\infty}=6.27$. Streamwise flow separation is evident near the symmetry plane. Flow leaving the nozzle quickly becomes overexpanded, and as it recompresses, the boundary layer separates from the body. The near-surface flow pattern is repeated for the lower exit pressure ratio cases, and the separation point moves progressively closer to the nozzle as the exit pressure ratio is reduced. This result is only a suggestion of the importance that offdesign performance analysis may assume. To take a step closer to design-point conditions, though, flow solutions are next investigated at a hypersonic Mach number.

\subsection{Generic Hypersonic Vehicle Flow Solutions at $M_{\infty}=6$}

With a sizeable body of numerical/experimental comparisons in already in hand, a purely numerical assay of hypersonic plume/afterbody flow fields is now presented. This set of calculations seeks to: 1) demonstrate the ability of the CFD code to solve power-on flow fields in the hypersonic regime, 2) explore the fluid dynamics of plume impingement at hypersonic speeds, and 3) investigate the benefits, if any, of thrust vectoring for propulsive lift. Regarding the latter, it has been proposed that thrust vectoring may be useful at hypersonic flight conditions to trim out very large pitching moments that are generated by propulsive forces (private communication from G. S. Deiwert, NASA Ames Research Center, Moffett Field, California).

Selecting flight conditions proved to be somewhat arbitrary. No experimental or flight test data are available for powered hypersonic flight as yet. For these numerical experiments, $M_{\infty}=6, R e=230,000$ $\mathrm{cm}^{-1}$ were chosen to mimic a possible cruise speed $(\approx 17,704 \mathrm{~m} / \mathrm{s})$ and altitude $(\approx 15 \mathrm{~km})$ of a hypersonic transport. Again, fully turbulent flow was assumed over the entire body. Nozzle exit conditions in this regime are equally vague, though exit pressure ratios on the order of 100 to 1000 have been spoken of. As 
such, the operating conditions for these calculations were chosen to be $p_{e} / p_{\infty}=100, M_{e}=2.04$. The quantity $\epsilon_{v}$ is now introduced to specify the angle of thrust vectoring, measured positive downward from the body axis.

4.6.1 Analysis of the exhaust plume/afterbody interaction- Pressure contours for the entire flow field at $M_{\infty}=6$ are presented in figure 34. The angle of attack, $\alpha$, is zero, and the thrust vector angle, $\epsilon_{v}$, is also zero in this solution. Formation of the wing shock is evident at the wing-body junction, and the plume shock is visible on the symmetry plane. The plume shock is clearly defined in figure 35 , and its shape approximates a conical surface. It interacts very strongly with the afterbody, and figure 36 demonstrates the effect it has on flow near the surface. The limiting streamlines on the afterbody reveal a complicated flow pattern. Postulated skin friction lines presented alongside the calculated limiting streamlines suggest multiple crossflow separations and reattachments, an interpretation of which is put forth here.

The intersection of the plume shock and afterbody induces crossflow separation $S_{3}$. The thickening boundary layer in advance of this separation generates compression waves that coalesce to form a weak shock upstream (in the crossflow sense) of the plume shock. The result is a lambda shock structure at the body, as depicted in figure 37. Referring back to figure 35, the upstream leg of the lambda shock is evident near the body. The upstream leg of the shock induces flow separation along line $S_{1}$ that rolls up into a vortex. Unfortunately, tracing particle motions in the vortex does not produce the tightly braided patterns seen in subsonic [54] and transonic [55] flows because the streamwise flow speed is so much larger than the crossflow speed in hypersonic applications. Nonetheless, the core is evident as a pressure trough in figure 37 , and a vorticity peak in figure 38 . The postulated crossflow pattern described here is sketched in figure 39.

The vortex produces a corner-like flow, driving secondary and tertiary vorticity peaks outboard of it. The secondary and tertiary vortices are not actually recirculating, but their influence on the body produces the reattachment $R_{1}$ and separation $S_{2}$. The large vortex attaches at $R_{2}$, just outboard of the strong leg of the plume shock. That shock causes flow separation at $S_{3}$, as mentioned before. As the underbody fairing tapers, expansion waves radiate from it and bring about reattachment at $R_{3}$. Finally, the symmetry plane becomes a separation point by necessity, though the limiting streamlines in figure 36 show only slight convergence toward this line. This interpretation of the flow field suggests that the conical afterbody fairing is only responsible for $R_{3}$ : the other separation and reattachment lines are a direct result of the plume shock interaction with the afterbody. Therefore, analogous patterns would be expected for other afterbody shapes under similar conditions.

4.6.2 Effect of thrust vectoring-It has already been observed that most of the scramjet thrust results from forces exerted at the exit plane of the nozzle. Because the nozzle is below the body centerline (the approximate location of the center of gravity), the thrust force contributes a large nose-up pitching moment. Trimming the vehicle with aerodynamic control surfaces becomes impractical; this would require large, heavy surfaces and would incur an excessive trim drag penalty. However, the pitching moment due to propulsion can be reduced by vectoring thrust from the nozzle downward. As figure 40 shows, thrust vectoring rotates the thrust vector toward the center of gravity, effectively reducing the lever arm from which this force acts. Thrust vectoring will also produce several potentially important auxiliary effects. Lift will increase due to the downward momentum flux from the nozzle, while thrust will decrease. The plume shock will be stronger because the external flow must turn more to accomodate the plume flow. Finally, the plume flow will expand more as it turns up the afterbody, giving rise to reduced afterbody 
forces. To determine the net result of these differences, solutions were obtained at $\epsilon_{v}=3^{\circ}$ and $\epsilon_{v}=10^{\circ}$, other conditions constant.

The variation of $c_{m y}$ with thrust vector angle is shown in figure 41 . As expected, thrust vectoring contributes a nose-down pitching moment. In contrast to the sensitivity of $c_{m,}$ to changes in exit pressure ratio discussed above, the afterbody forces have little effect on moments induced by thrust vectoring. Being able to control the vehicle balance in this way may be important to ensure good handling qualities. Auxiliary effects of thrust vectoring on lift and thrust are shown in Table 4 . At the relatively small vectoring angles, the thrust is not seriously degraded, while lift nearly doubles in the interval from $\epsilon_{v}=0^{\circ}$ to $\epsilon_{v}=10^{\circ}$.

Table 4. Vehicle lift and thrust with thrust vectoring.

$$
M_{\infty}=6, \alpha=0^{\circ}, p_{e} / p_{\infty}=100 \text {. }
$$

\begin{tabular}{|c|c|c|c|}
\hline$\epsilon_{v}$ & $0^{\circ}$ & $3^{\circ}$ & $10^{\circ}$ \\
\hline \hline$c_{T}$ & 0.1173 & 0.1171 & 0.1139 \\
\hline$c_{L}$ & 0.0156 & 0.0202 & 0.0307 \\
\hline
\end{tabular}

Thrust vectoring also deflects flow away from the afterbody. Figure 42 compares limiting streamlines on the afterbody for $\epsilon_{v}=0^{\circ}$ and $\epsilon_{v}=10^{\circ}$. The afterbody flow pattern, though topologically similar to the $\epsilon_{v}=0^{\circ}$ solution, exhibits less turning of the flow near the body, weakening the exhaust plume/afterbody interaction.

\subsection{Summary}

At this point, numerous flow solutions have been presented for different geometries and flow conditions. Comparisons have been made with experimental data, sensitivities to various parameters have been assessed, and interpretations of the flow fields have been made. Any one of these flow solutions contains a wealth of information - more than can be analyzed even within the scope of a single paper. The goal in this work has been to extract only those aspects of the flow field phenomena that pertain directly to the problem at hand. This chapter has addressed those issues in the context of practical flight conditons about a realistic geometry. Now it is appropriate to put this work in perspective; to draw more general conclusions about the work and its results, and to look for new directions for future work. 


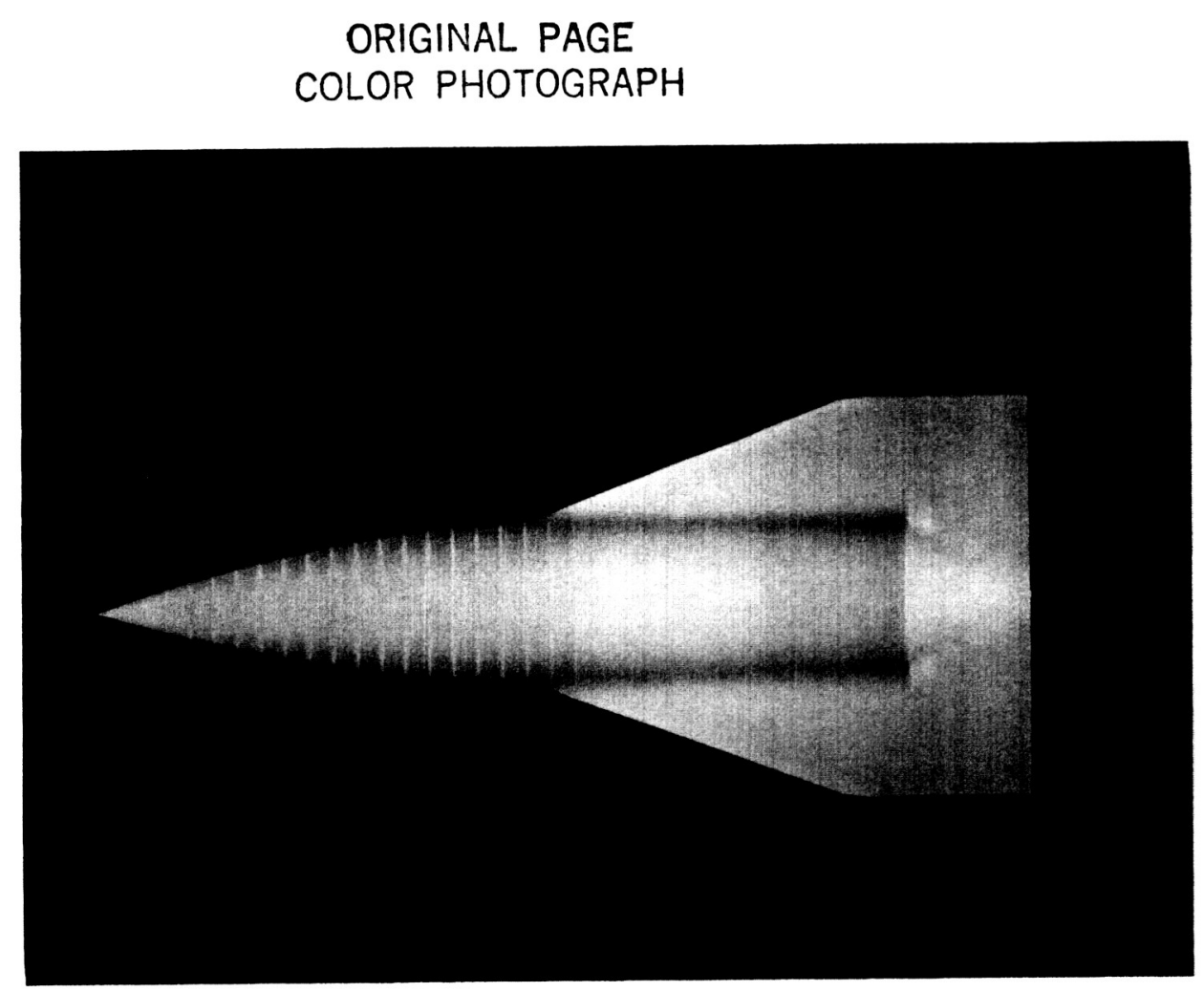

(a) top view

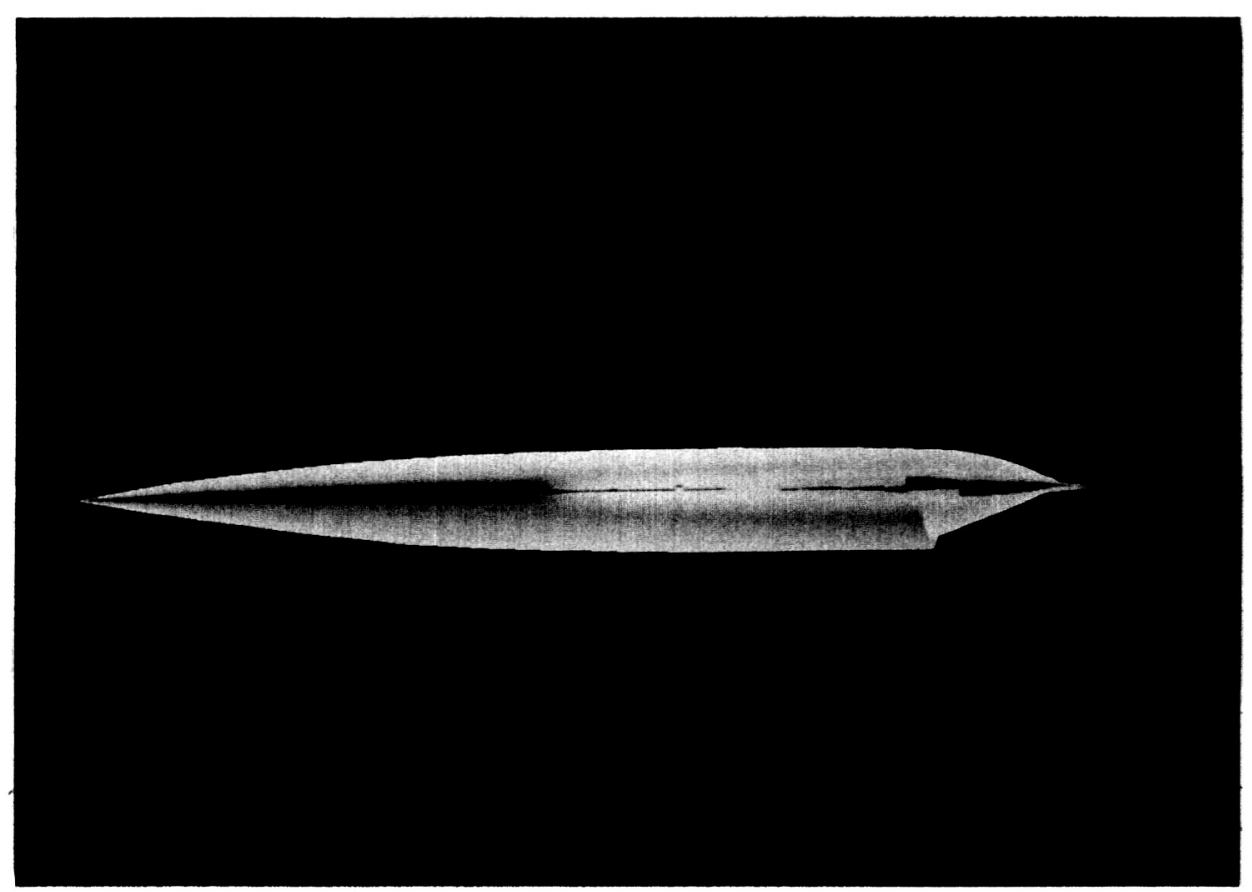

(b) side view

Figure 25. Generic hypersonic vehicle geometry. 


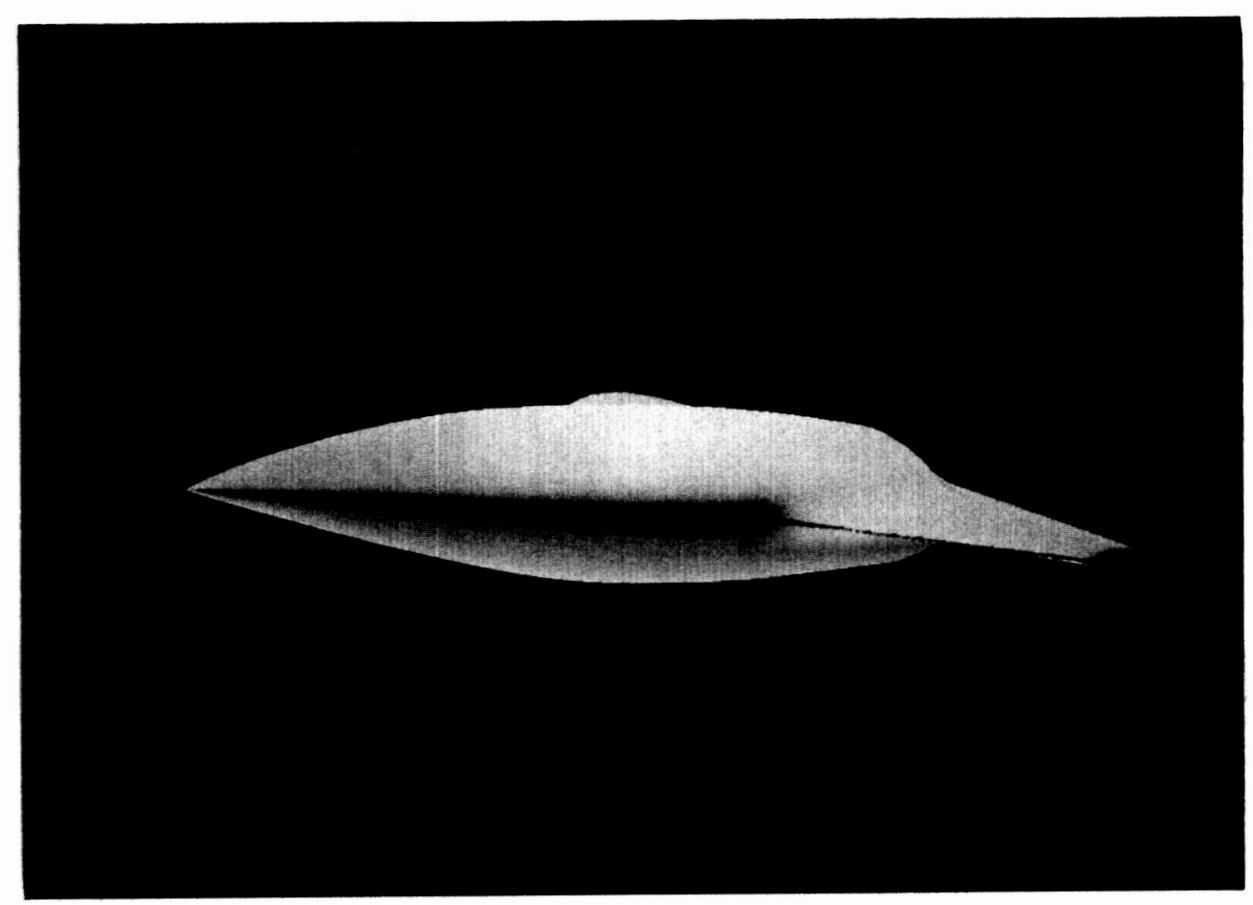

(c) front three-quarter view

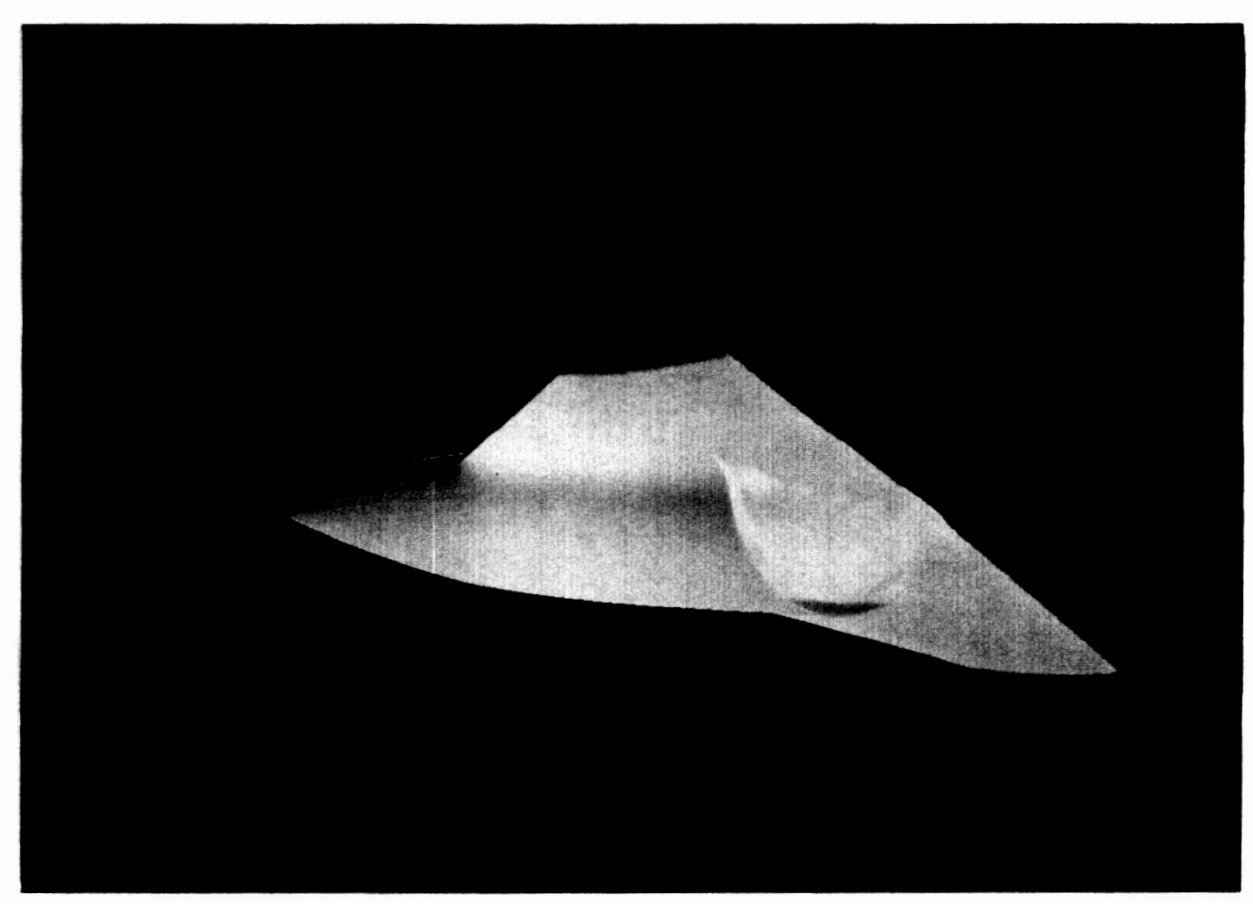

(d) rear three-quarter view

Figure 25. Generic hypersonic vehicle geometry, concluded. 


$$
\begin{aligned}
& \text { ORIGINAL PAGE } \\
& \text { COLOR PHOTOGRAPH }
\end{aligned}
$$

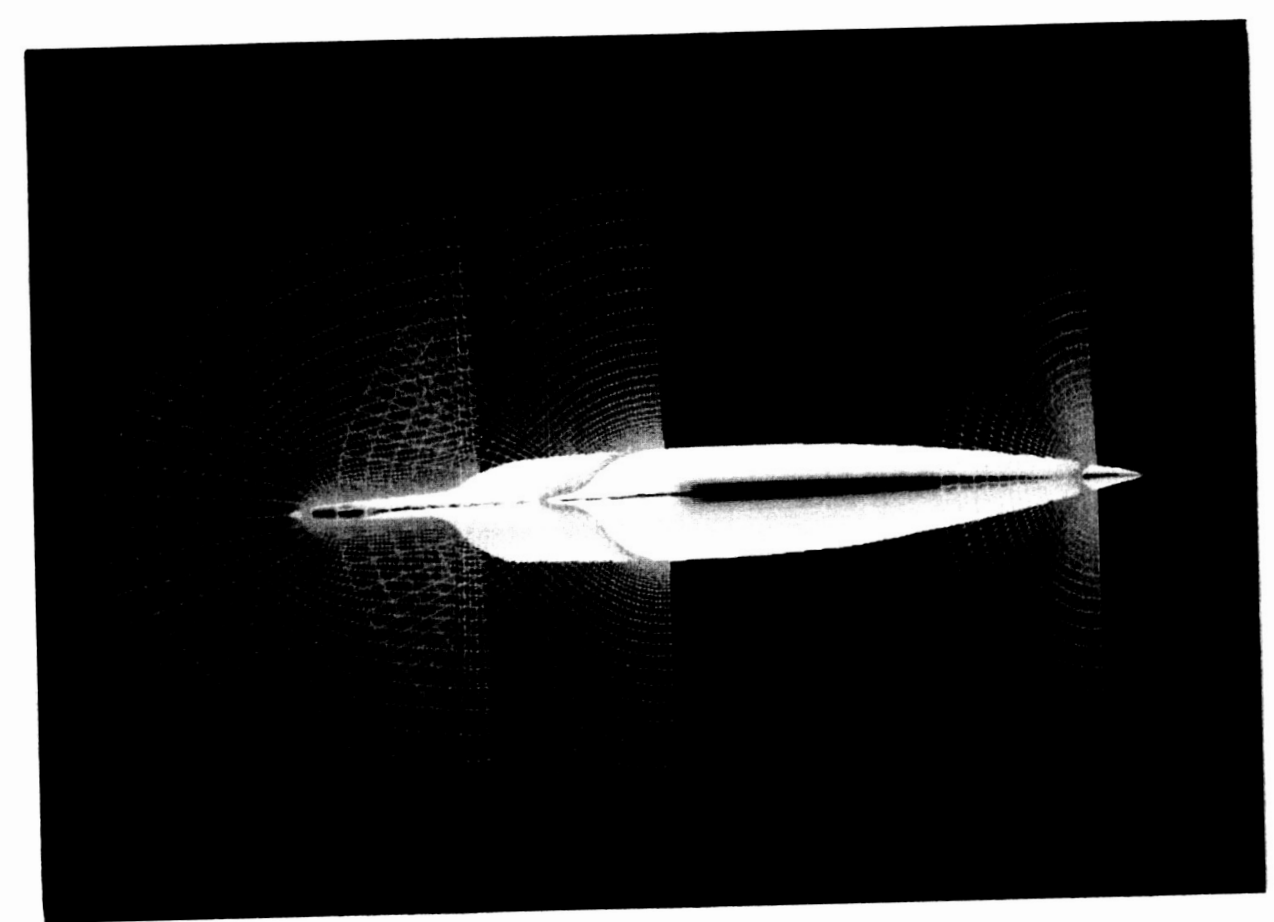

Figure 26. O-H grid topology for generic hypersonic vehicle. 


\section{ORIGINAL PAGE \\ COLOR PHOTOGRAPH}

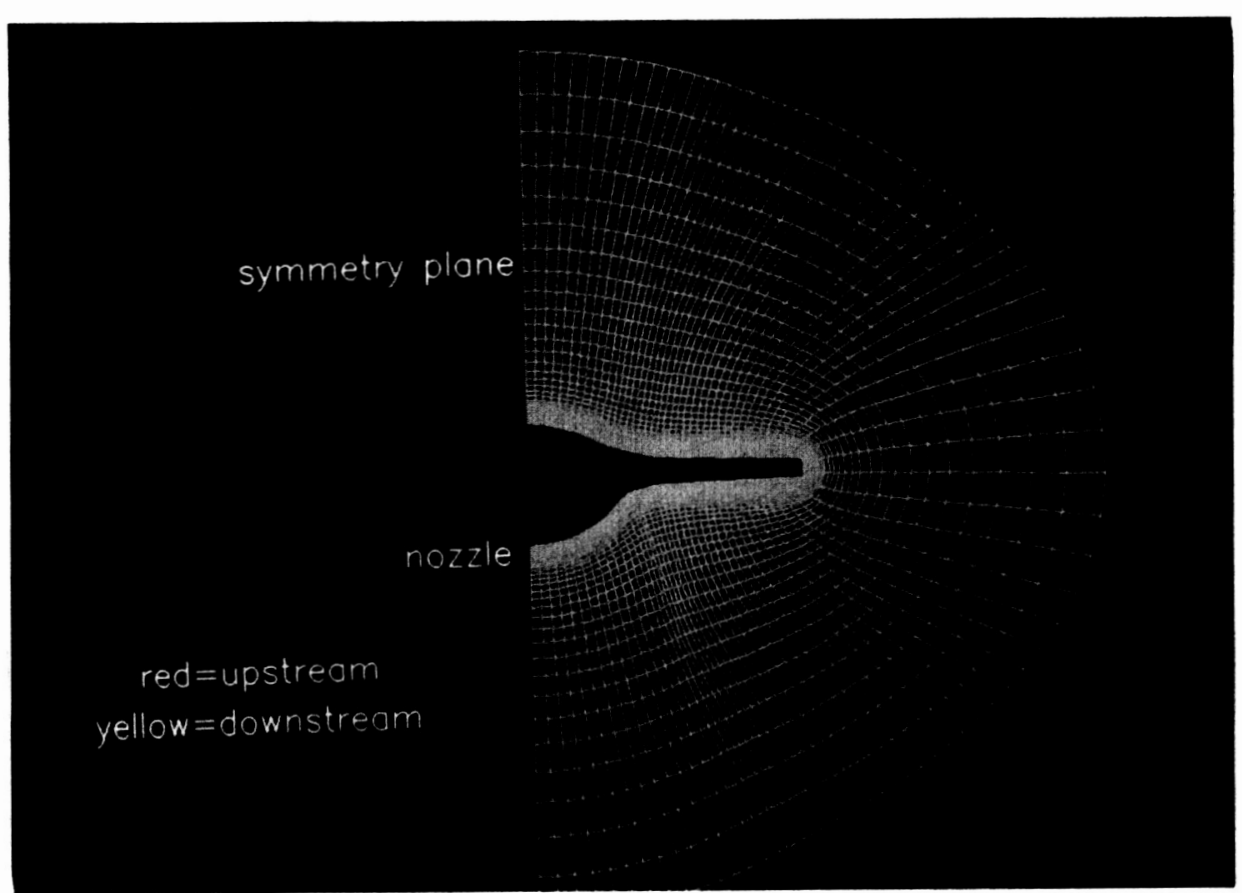

(a) overall view

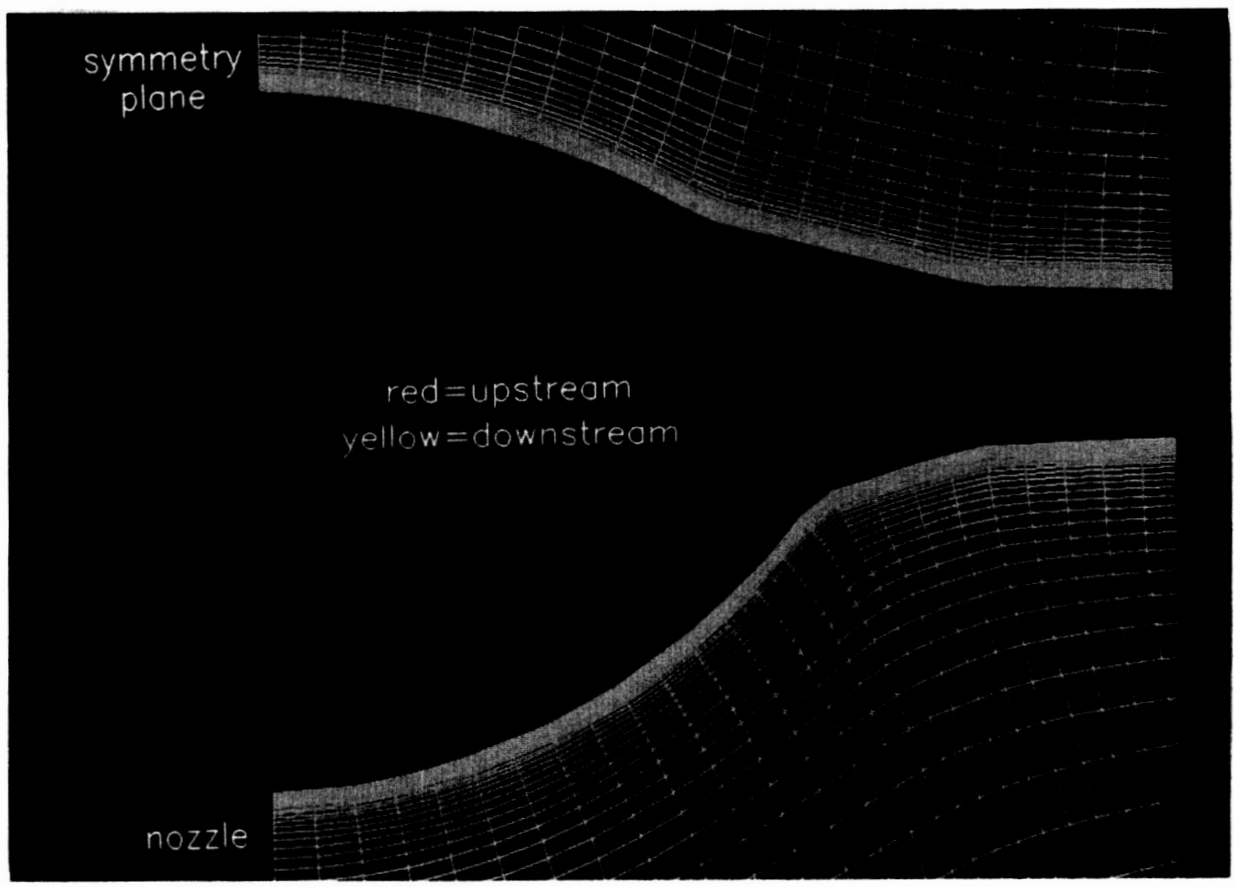

(b) detailed view near nozzle exit

Figure 27. Zonal interface at nozzle exit plane of generic hypersonic vehicle. 


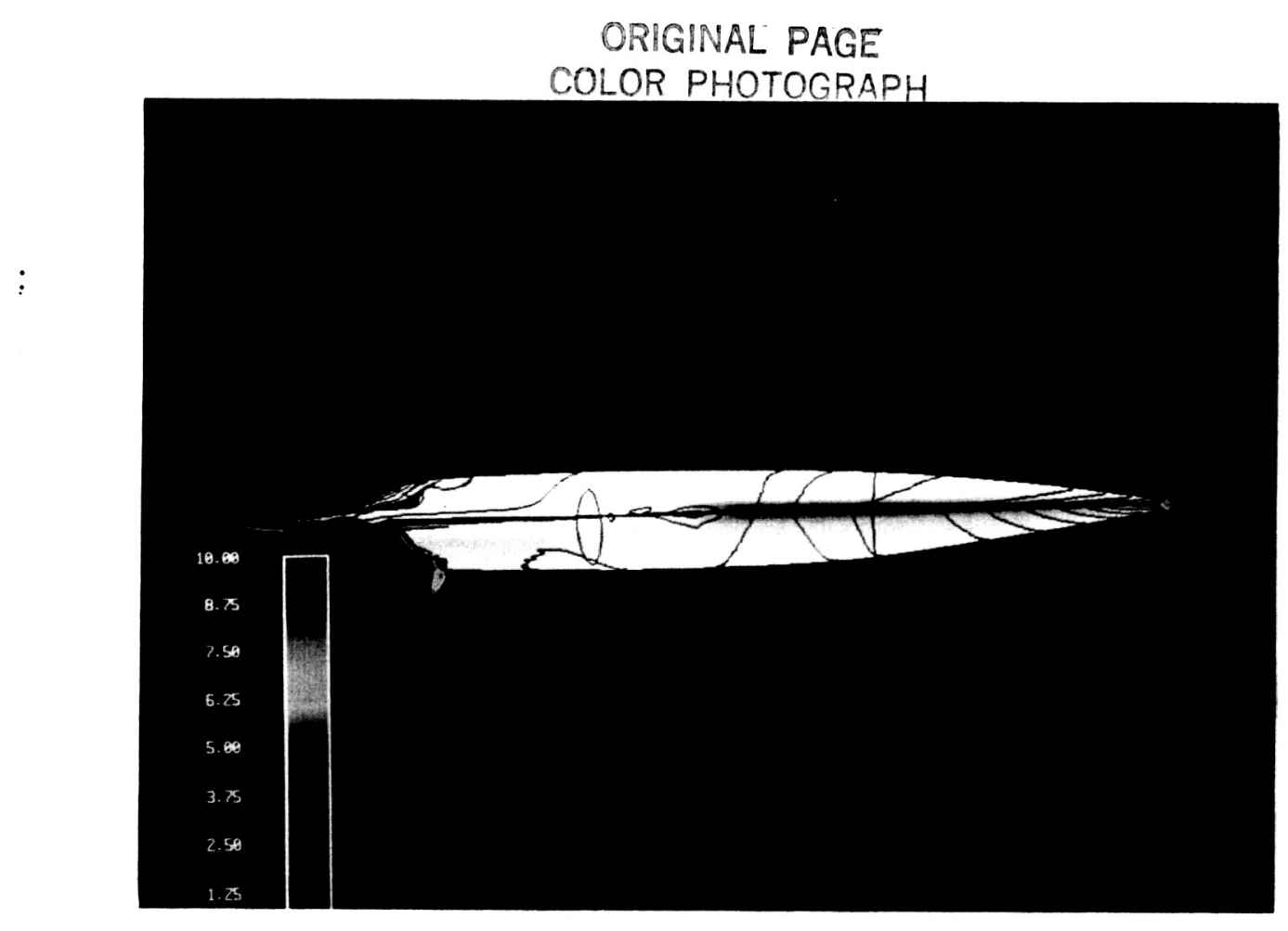

Figure 28. Perspective view of pressure contours on surface and selected grid planes of the generic hypersonic vehicle. $M_{\infty}=1.46, \quad \alpha=0^{\circ}$, $p_{e} / p_{\infty}=6.27$.

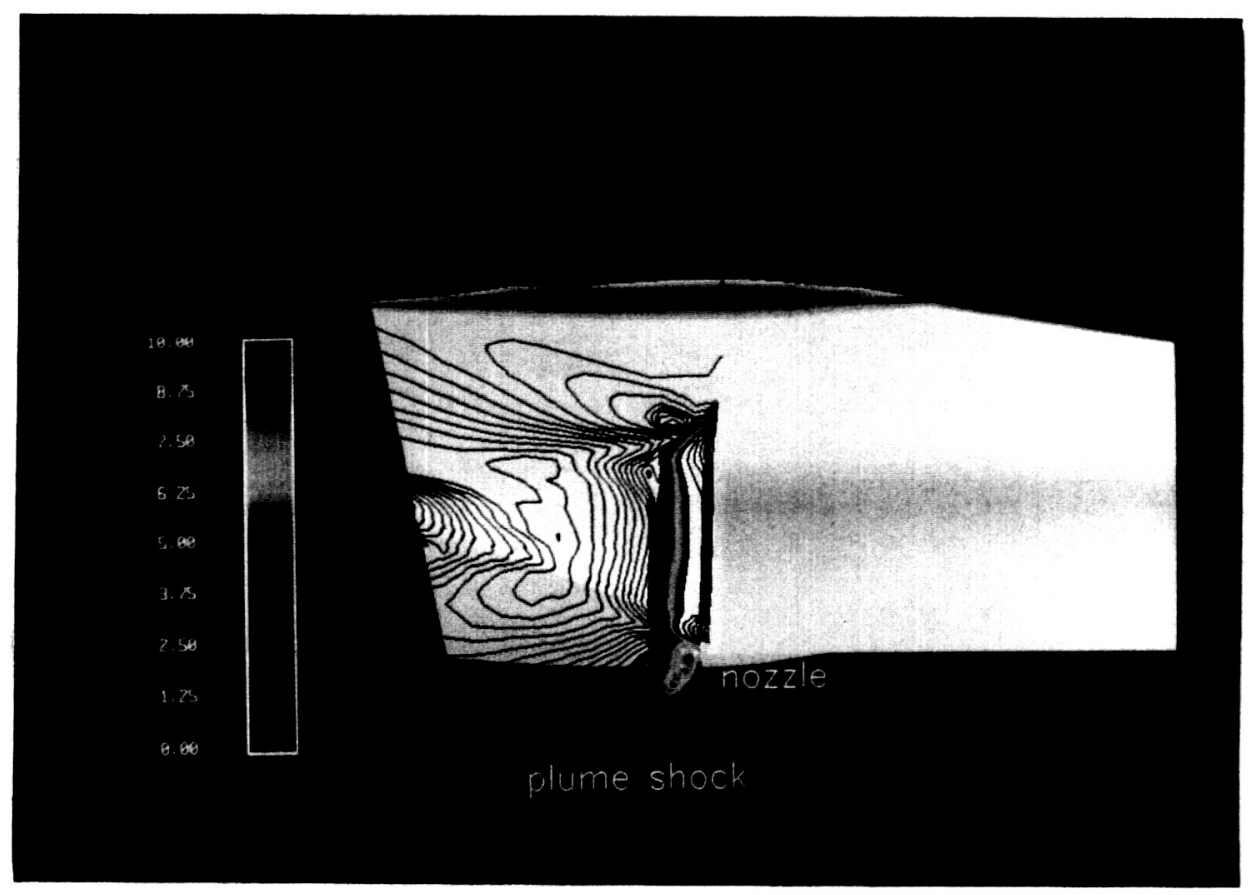

Figure 29. Detailed view of pressure contours on generic hypersonic vehicle near nozzle exit. $M_{\infty}=1.46, \quad \alpha=0^{\circ}, \quad p_{e} / p_{\infty}=6.27$. 

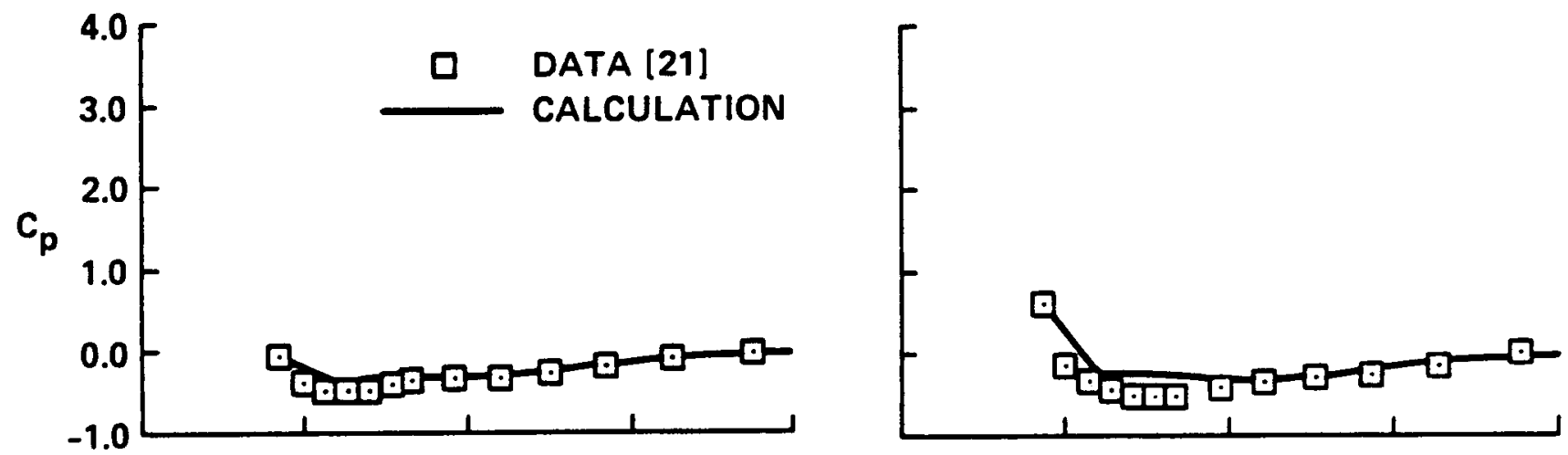

(a) $p_{e} / p_{\infty}=0.88$

(b) $p_{e} / p_{\infty}=1.92$
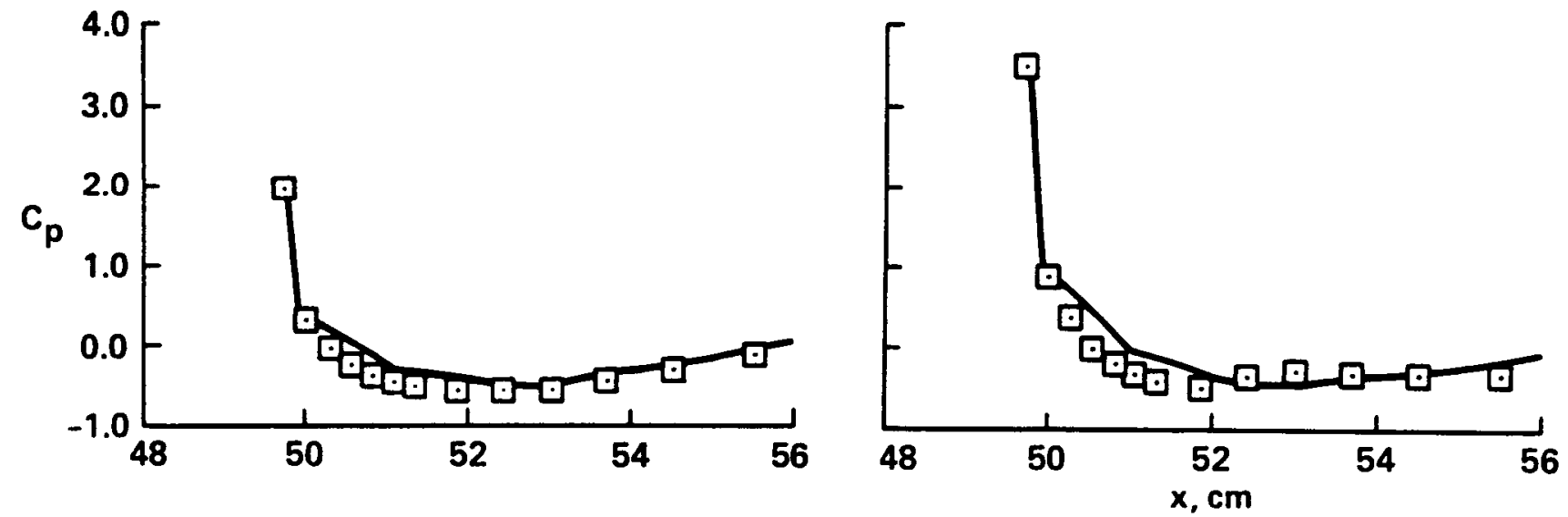

(c) $p_{e} / p_{\infty}=3.97$

(d) $p_{e} / p_{\infty}=6.27$

Figure 30. Comparison of calculated and experimental surface pressure coefficients on windward symmetry plane of generic hypersonic vehicle downstream of nozzle exit. $M_{\infty}=1.46, \alpha=0^{\circ}$. 


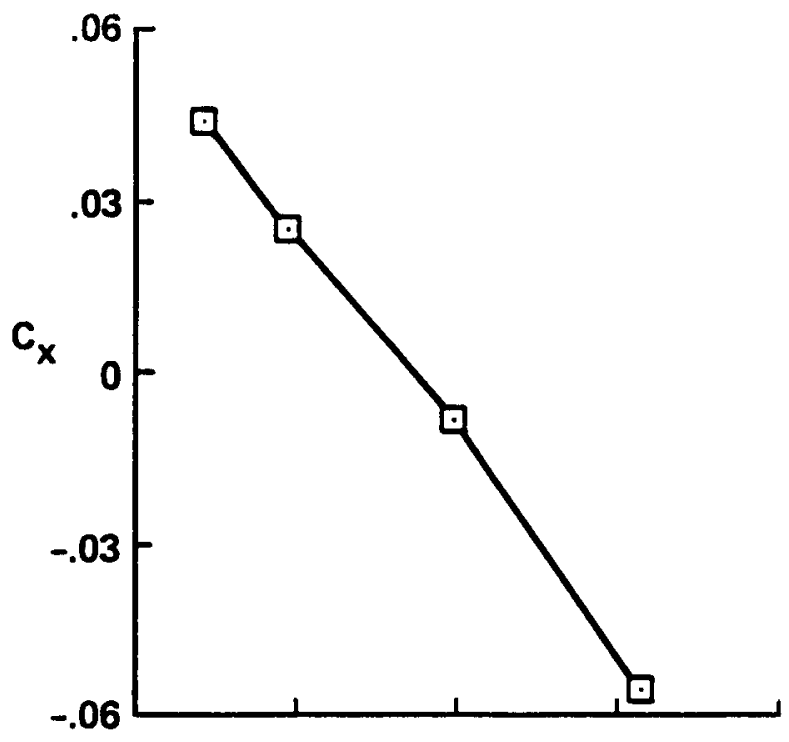

(a) $x$-force coefficient

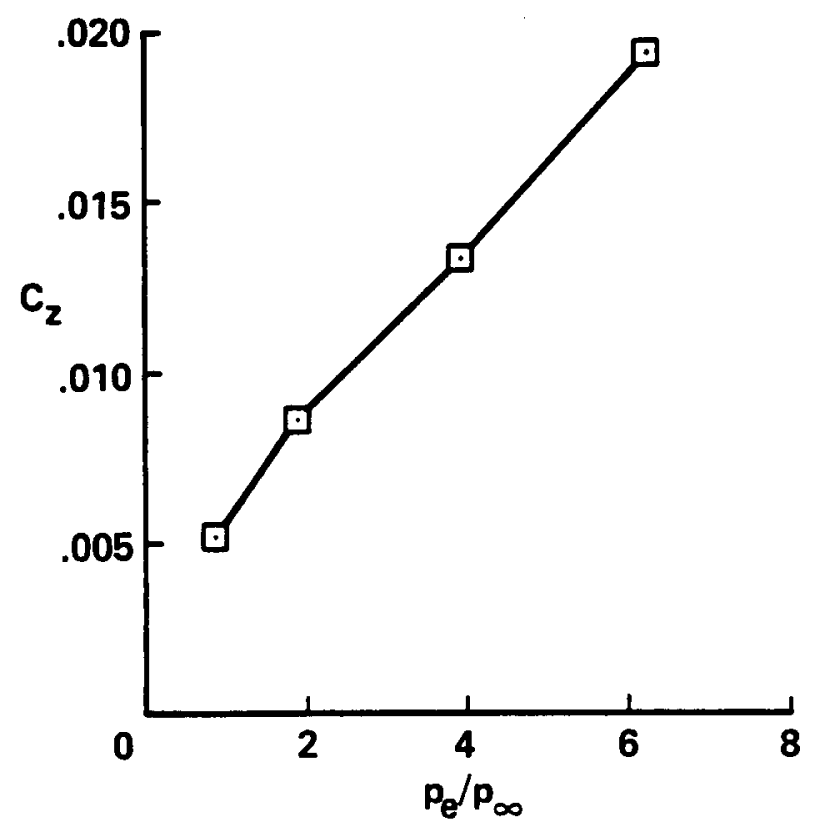

(b) $z$-force coefficient

Figure 31. Variation of force coefficients with exit pressure ratio for generic hypersonic vehicle. $M_{\infty}=1.46, \quad \alpha=0^{\circ}$. 


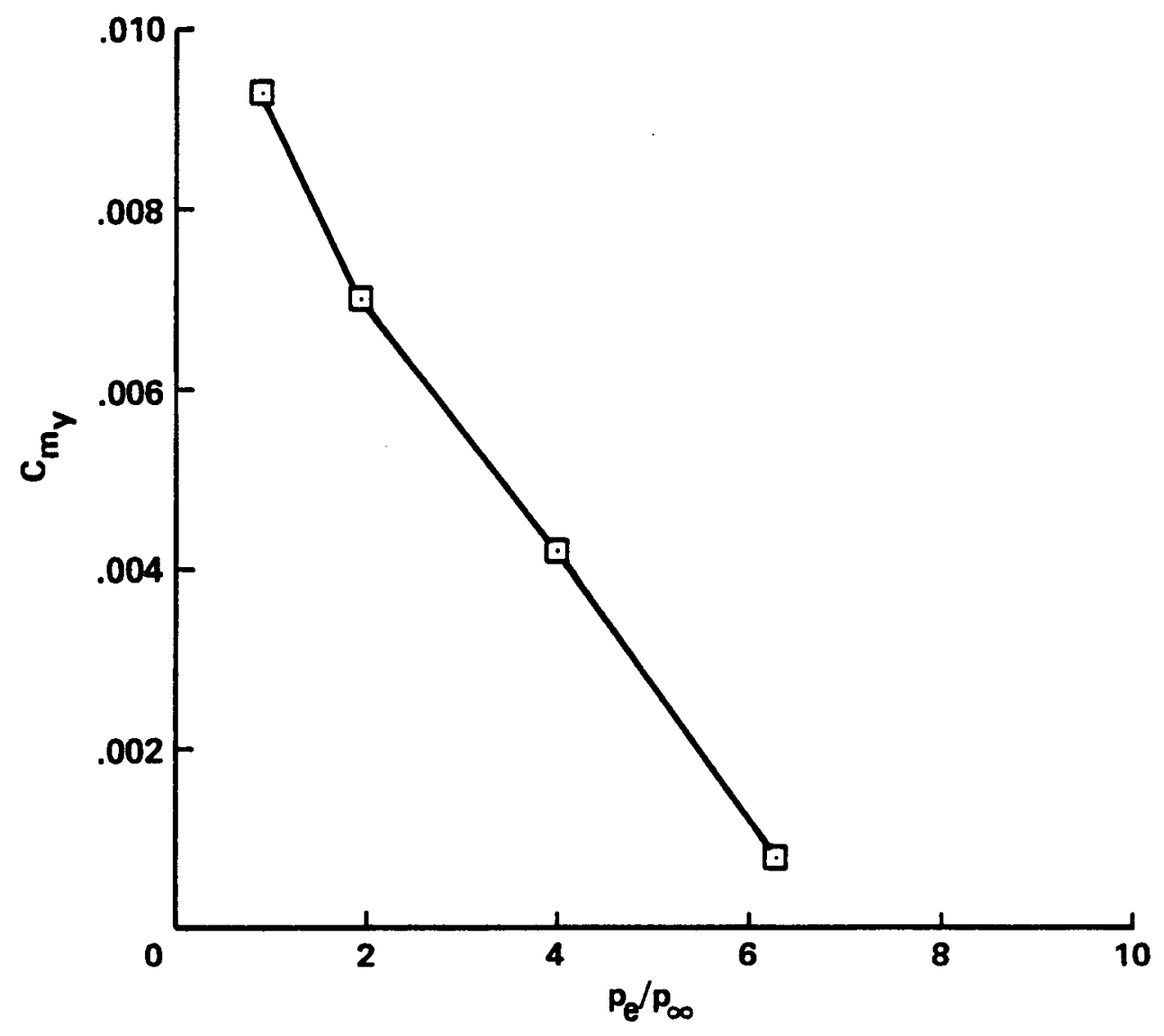

Figure 32. Variation of pitching moment with exit pressure ratio for generic hypersonic vehicle. $M_{\infty}=1.46, \alpha=0^{\circ}$. 


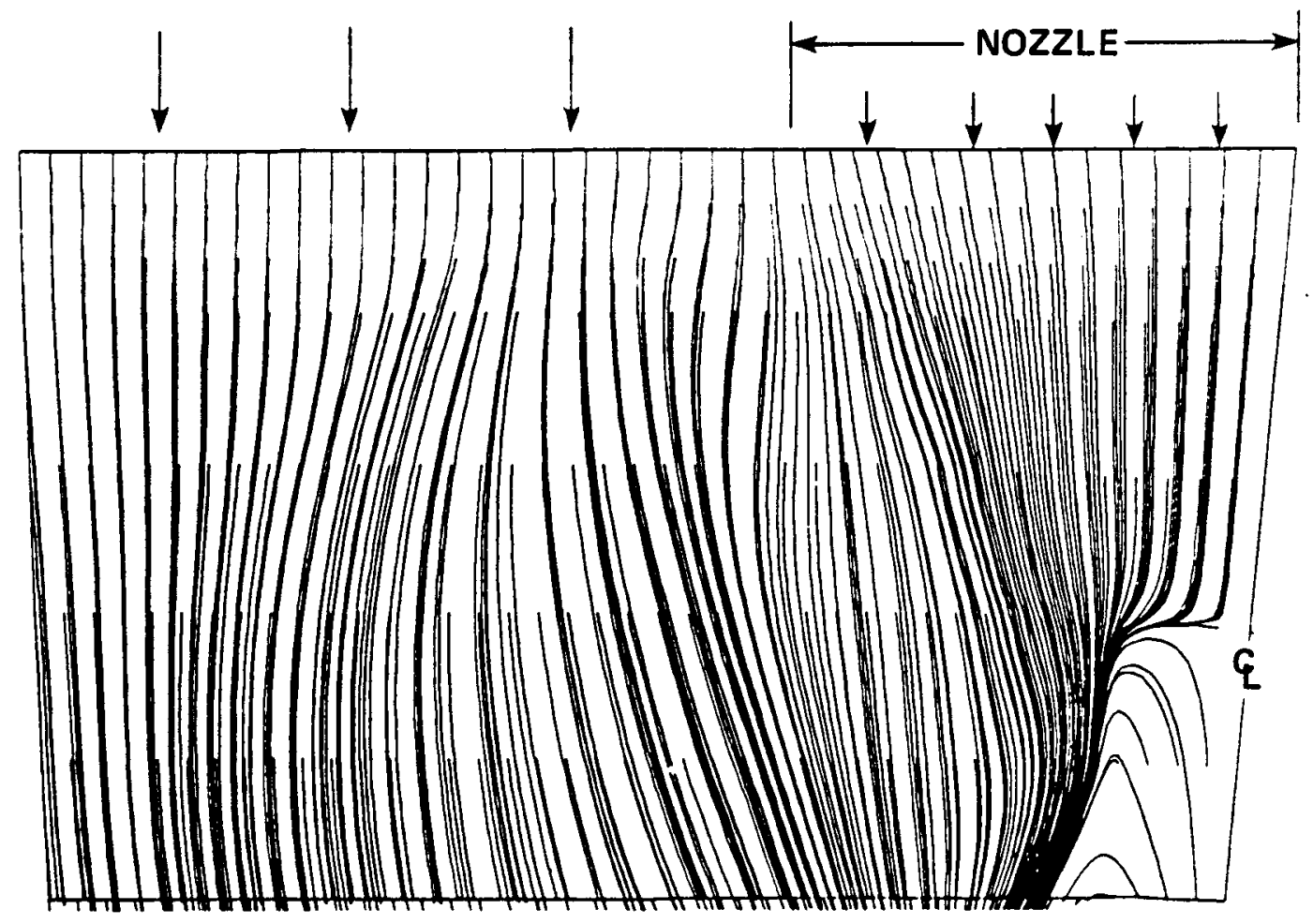

(a) calculated limiting streamlines

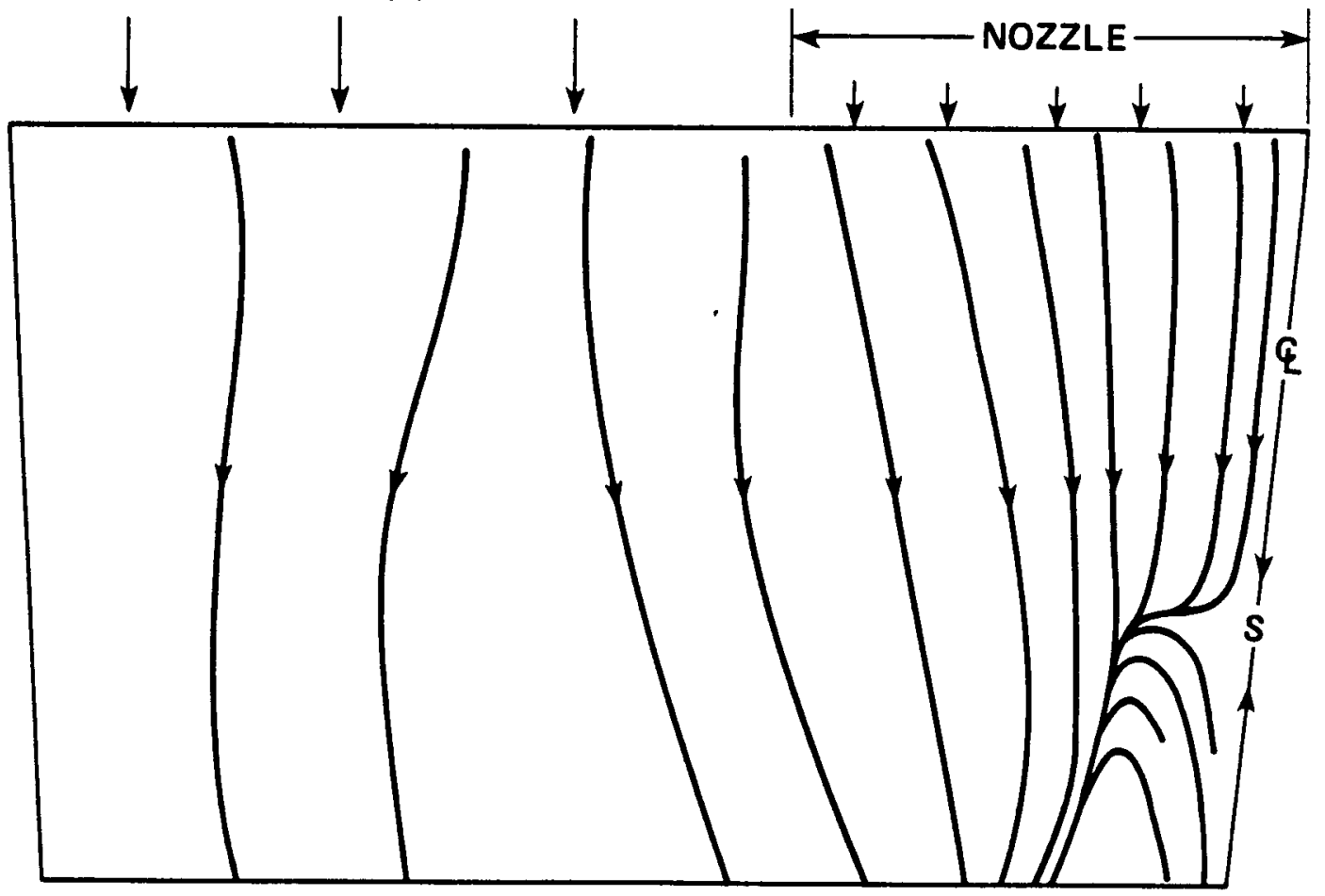

(b) postulated skin friction lines

Figure 33. Surface flow pattern on generic hypersonic vehicle afterbody. $M_{\infty}=1.46, \alpha=0^{\circ}, \quad p_{e} / p_{\infty}=6.27$. 


\section{ORIGINAL PAGE \\ COLOR PHOTOGRAPH}

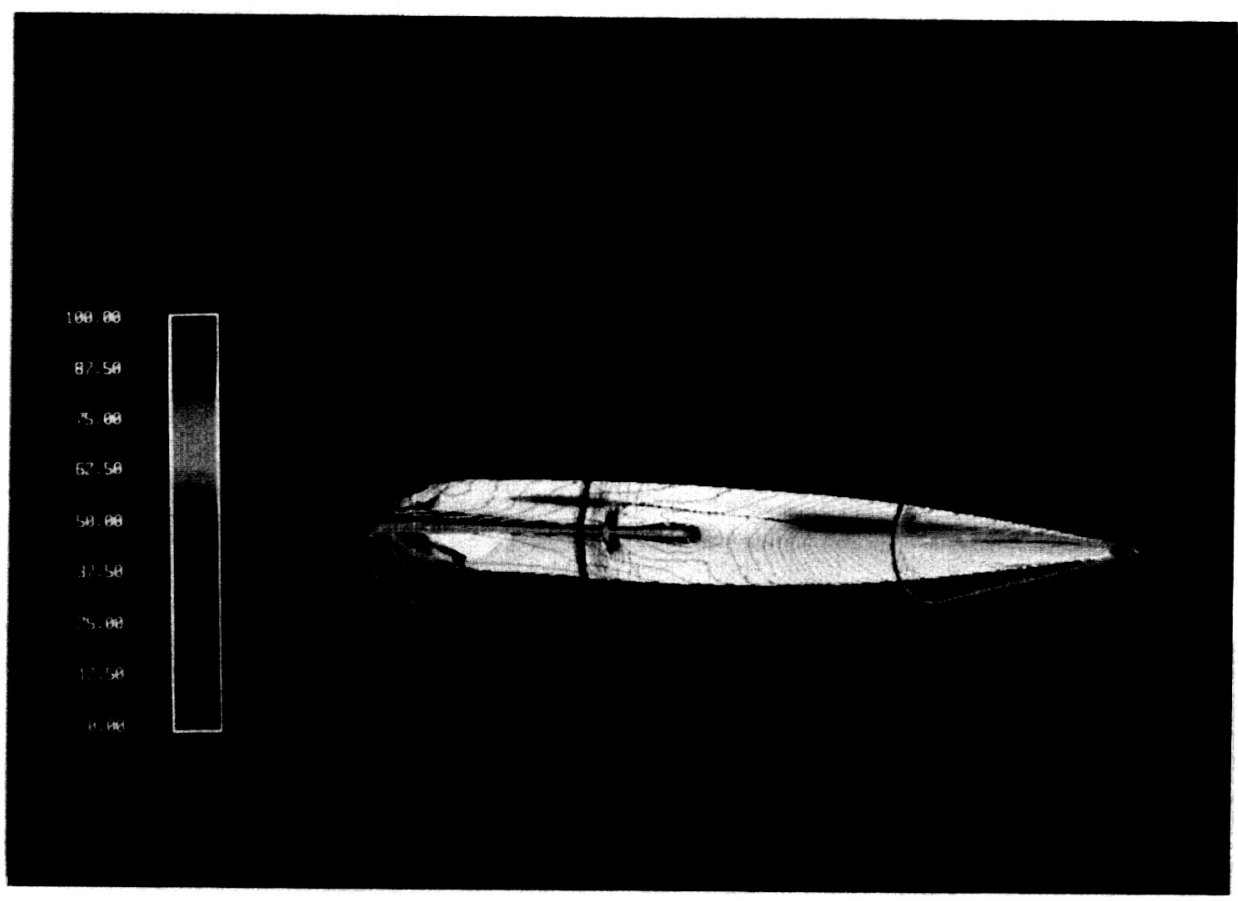

Figure 34. Perspective view of pressure contours on surface and selected grid planes of the generic hypersonic vehicle. $M_{\infty}=6, \alpha=0^{\circ}$, $p_{e} / p_{\infty}=100$.

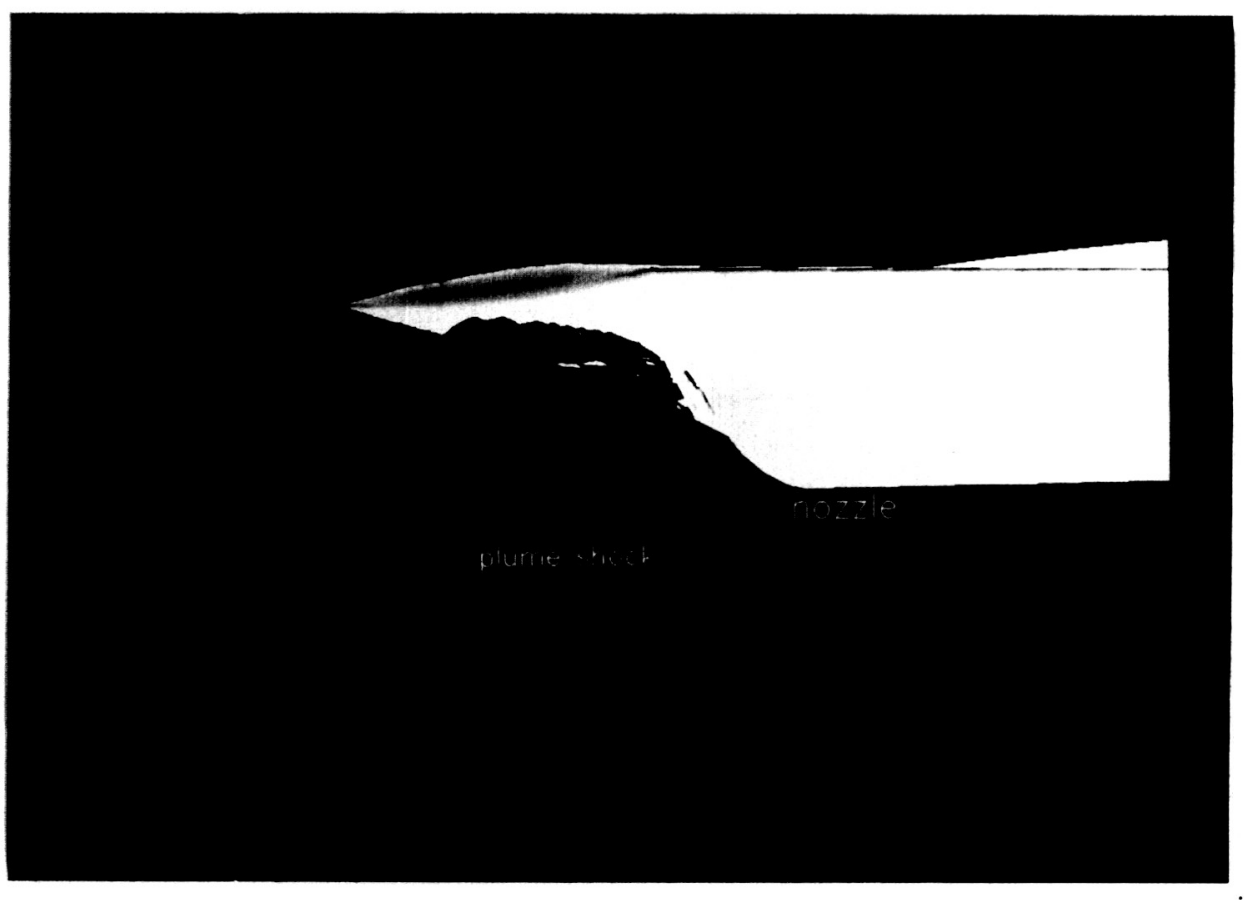

Figure 35. Shaded-surface representation of exhaust plume shock for generic hypersonic vehicle. $M_{\infty}=6, \quad \alpha=0^{\circ}, \quad p_{e} / p_{\infty}=100$. 


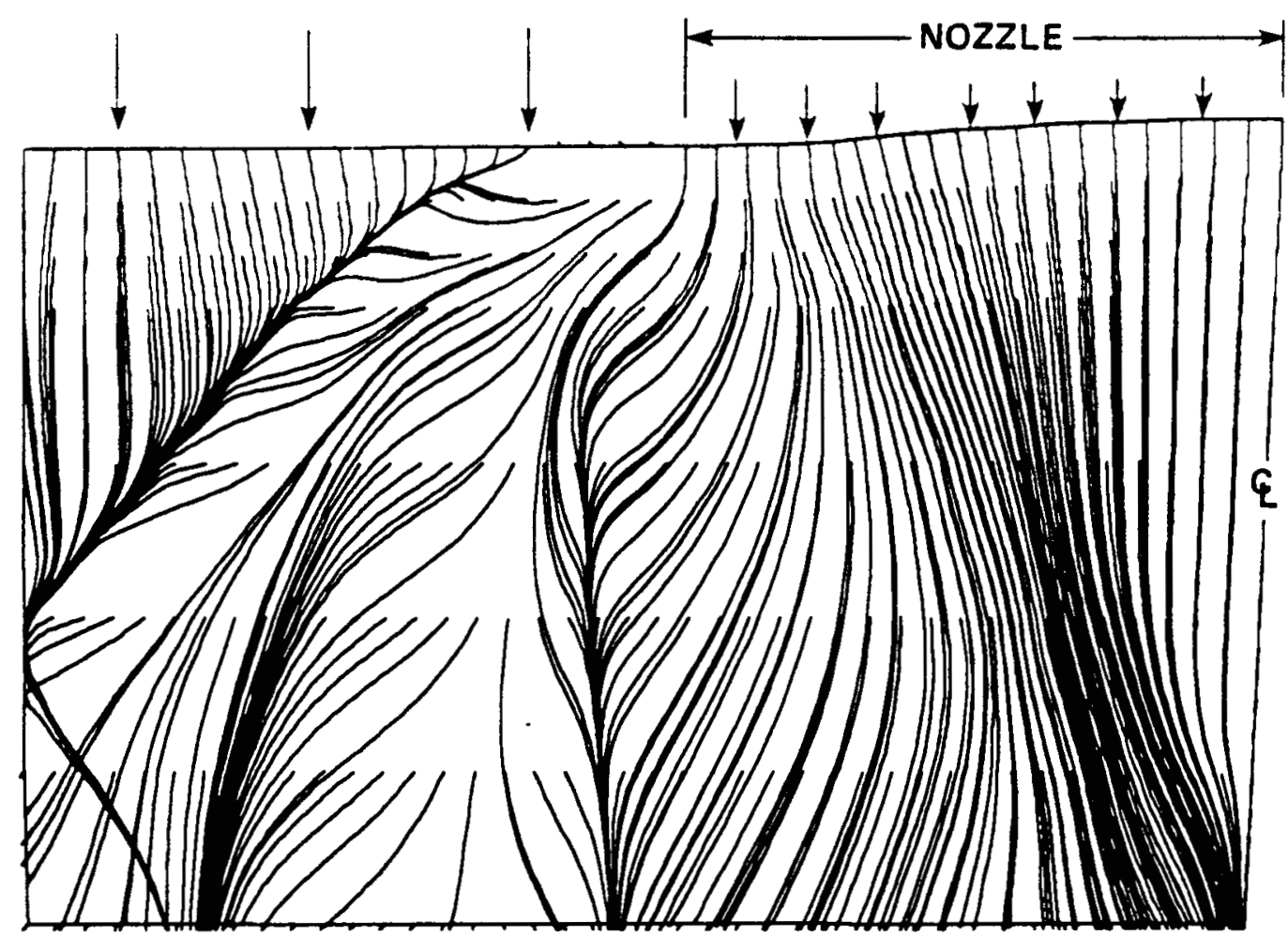

(a) calculated limiting streamlines

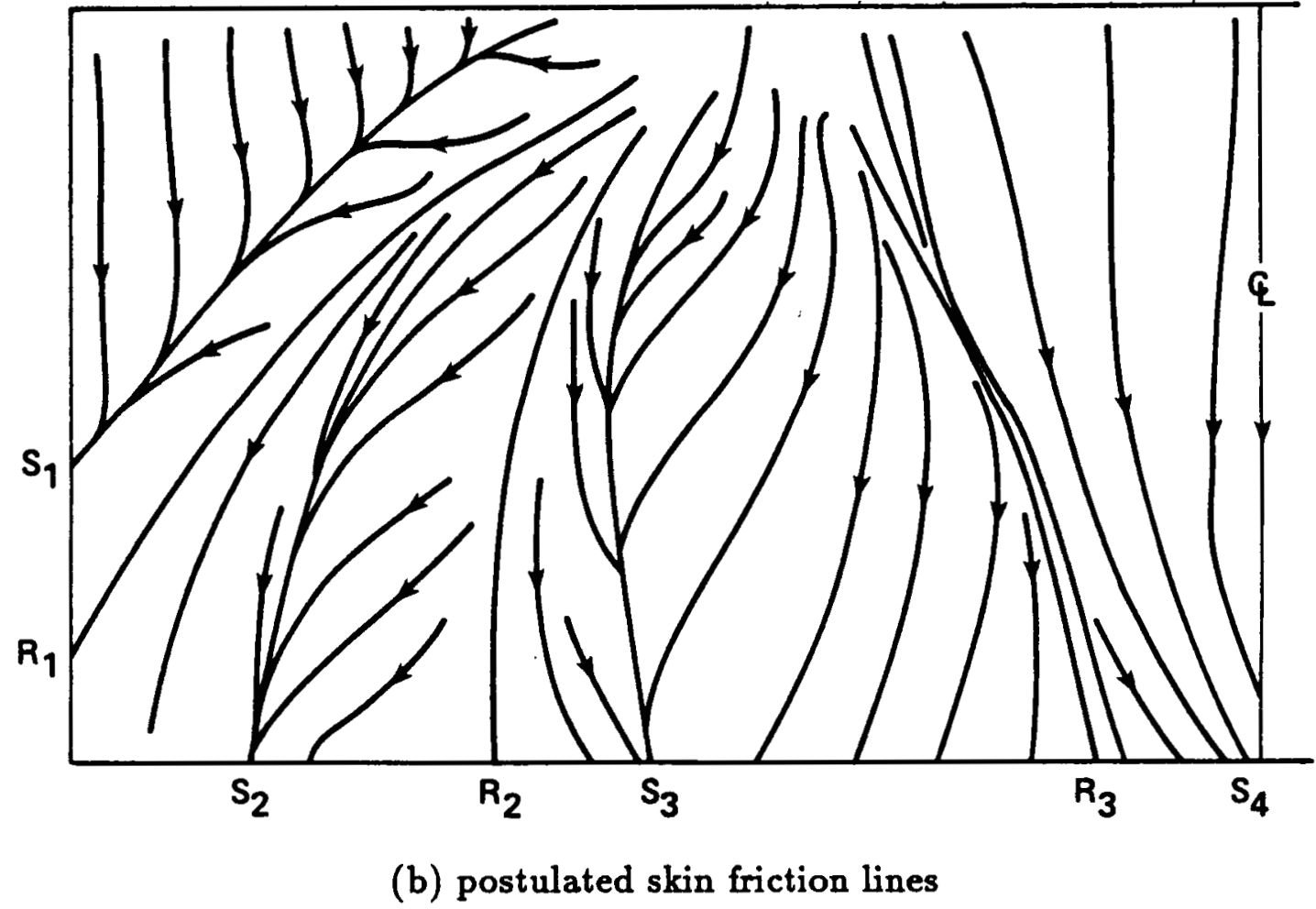

Figure 36. Surface flow pattern on generic hypersonic vehicle afterbody. $M_{\infty}=6, \alpha=0^{\circ}, \quad p_{e} / p_{\infty}=100$. 


\section{ORIGINAL PAGE \\ COLOR PHOTOGRAPH}

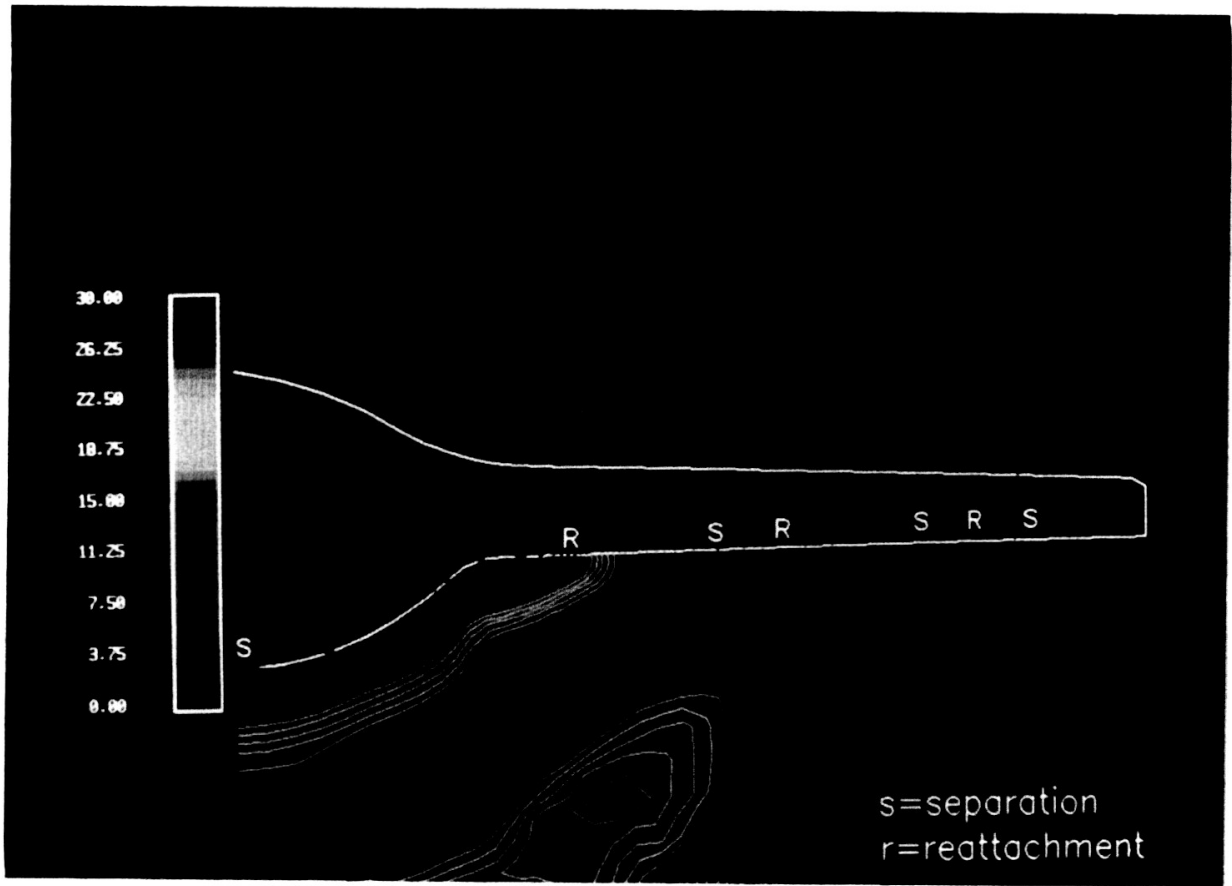

Figure 37. Crossflow pressure contours for generic hypersonic vehicle at $x=45 \mathrm{~cm} . M_{\infty}=6, \quad \alpha=0^{\circ}, \quad p_{e} / p_{\infty}=100$.

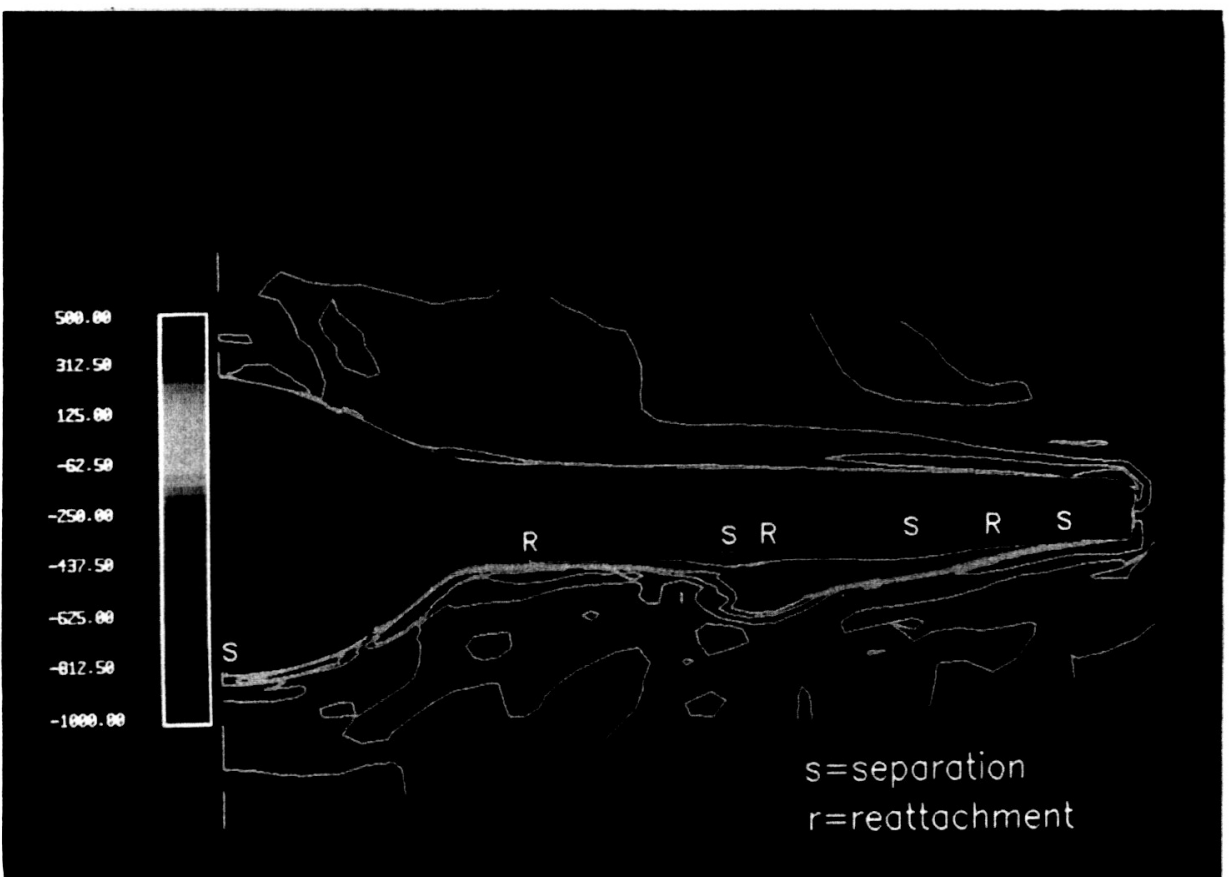

Figure 38. Crossflow vorticity ( $x$-component of vorticity vector) for generic hypersonic vehicle at $x=45 \mathrm{~cm} . M_{\infty}=6, \quad \alpha=0^{\circ}, \quad p_{e} / p_{\infty}=100$. 


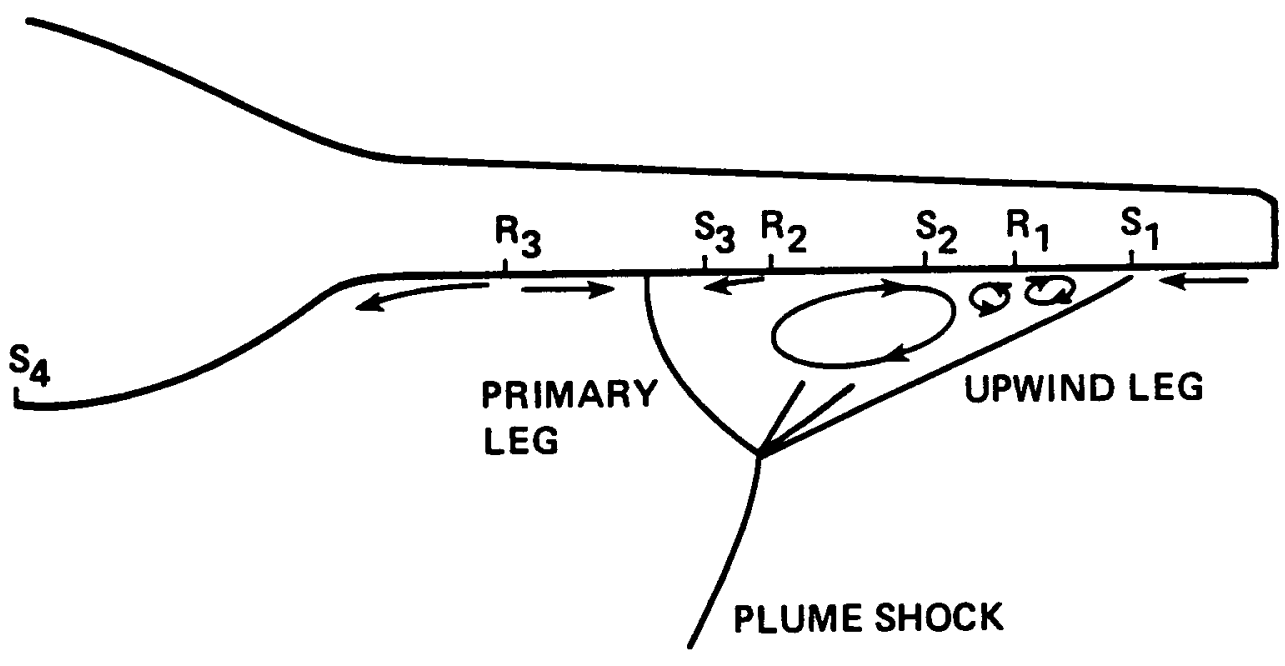

Figure 39. Postulated crossflow pattern for generic hypersonic vehicle at $x=45 \mathrm{~cm}$. 


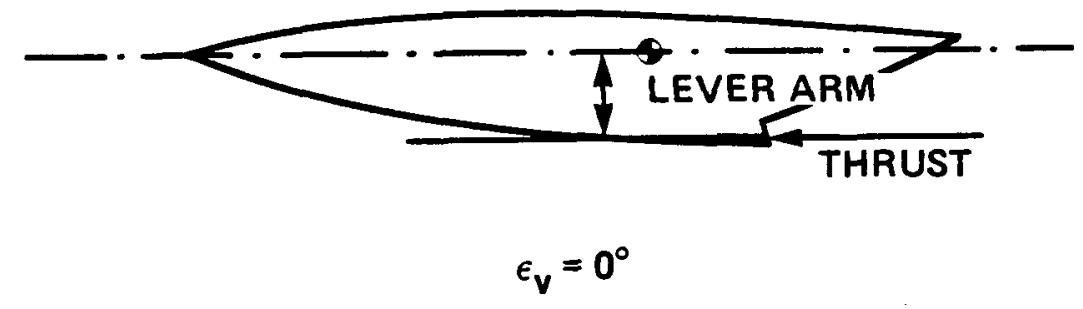

(a) without thrust vectoring

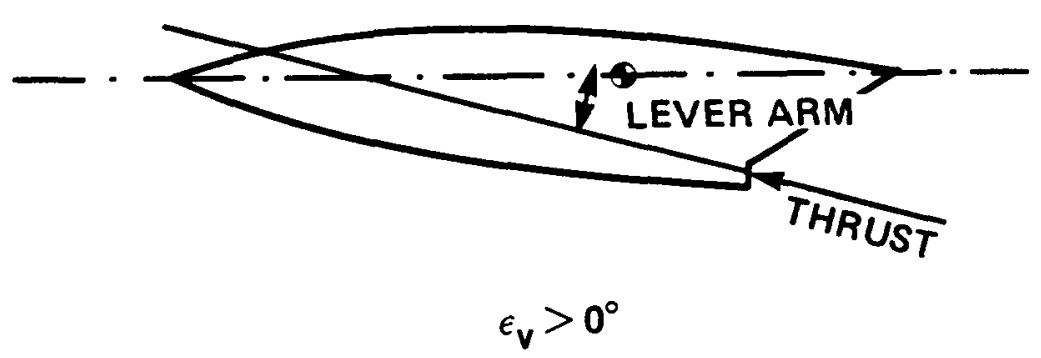

(b) with thrust vectoring

Figure 40. Schematic demonstrating the effect of thrust vectoring on vehicle balance. 


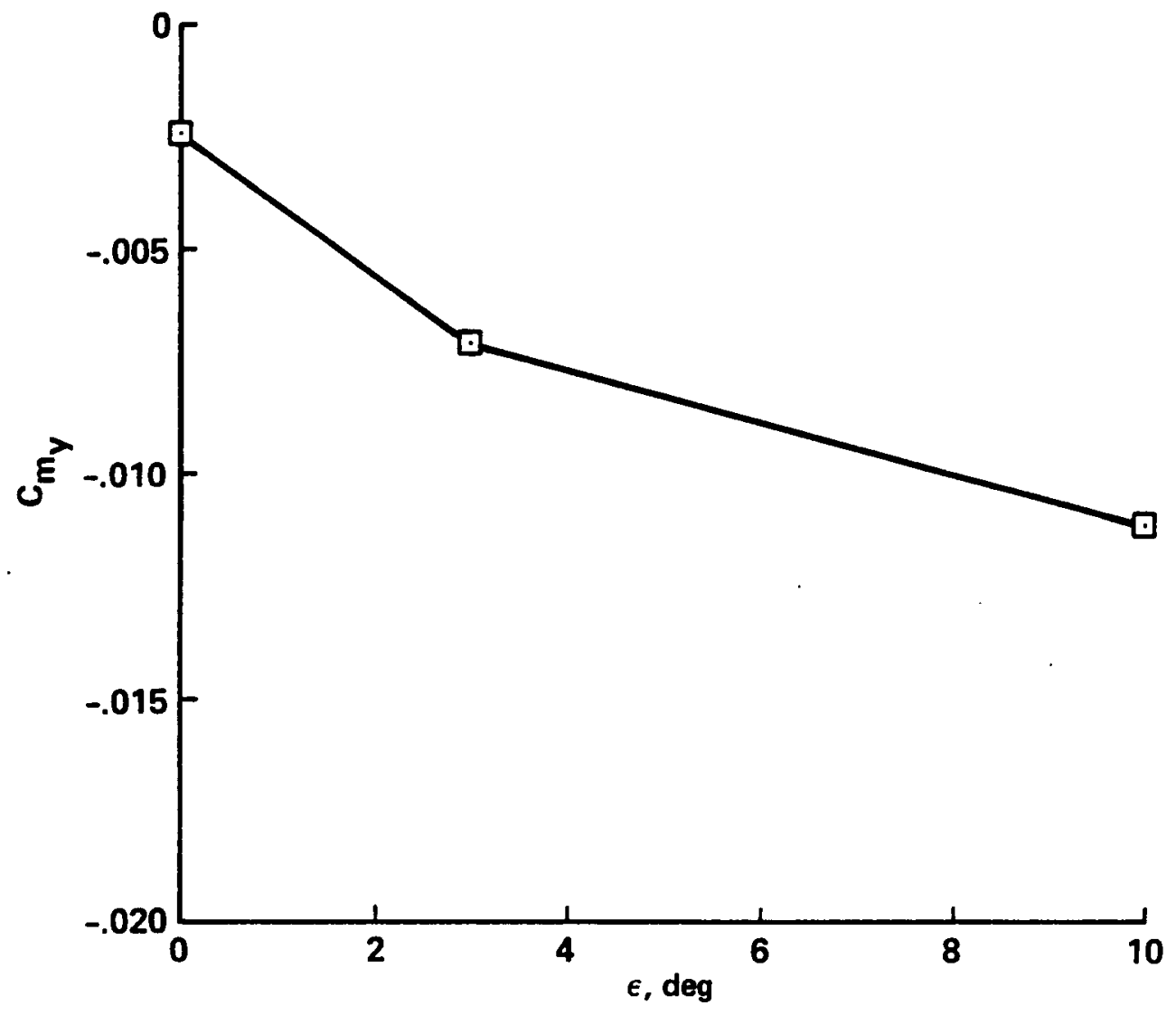

Figure 41. Variation of pitching moment with thrust vector angle $\epsilon_{v}$ for generic hypersonic vehicle. $M_{\infty}=6, \quad \alpha=0^{\circ}, \quad p_{e} / p_{\infty}=100$. 


\section{ORIGINAL PAGE \\ COLOR PHOTOGRAPH}

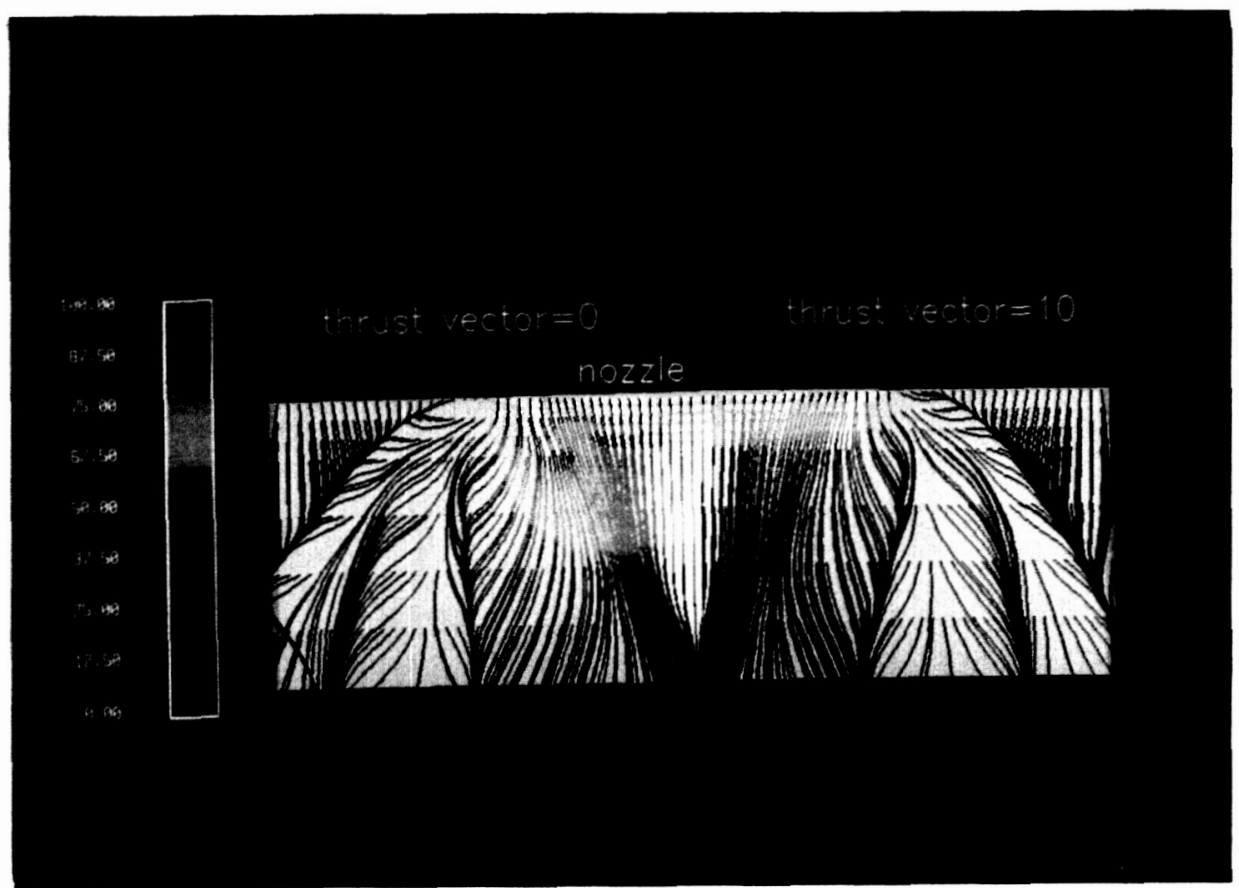

Figure 42. Comparison of calculated limiting streamlines colored by pressure on generic hypersonic vehicle afterbody with and without thrust vectoring. $M_{\infty}=6, \quad \alpha=0^{\circ}, \quad p_{e} / p_{\infty}=100$. 


\section{CHAPTER FIVE}

\section{CONCLUSION}

\subsection{Summary}

The objective of this investigation was to demonstrate a CFD capability to solve hypersonic flows involving exhaust plume/afterbody interaction. The UWIN code was calibrated with all available experimental data, and was then extended to conditions of practical interest. Some important parameters associated with scramjet propulsion were identified and studied based on the computational results. The sensitivity of performance to parametric variations was explained in terms of the underlying fluid dynamic phenomena. Some of the first power-on flow solutions have been obtained at hypersonic speeds for a realistic geometry. The impact of afterbody forces on vehicle balance was assessed, and the complex plume expansion process has been studied from a fluid dynamic perspective.

The nozzle/afterbody model brought out many features characteristic of exhaust plume flows. Complex flow patterns arise from interference of adjacent axisymmetric nozzles, leading to a highly nonuniform pressure distribution and recirculating flow in the footprint region. Variations in afterbody forces with exit pressure ratio, upsweep angle, exhaust gas isentropic exponent, and nozzle geometry were assessed, and comparisons with experimental data were generally good for these solutions. The experimental comparisons made in this study instilled confidence in the physical correctness of the calculations. Attention focused on what more could be learned about the problem from the detailed and voluminous information available from a CFD solution.

Solutions for the generic hypersonic vehicle demonstrated some of the first tip-to-tail flow field solutions at supersonic and hypersonic Mach numbers for power-on flows. Numerical/experimental comparisons again showed very good correlation. Flow field analysis of the supersonic results went on to reveal streamwise separation on the afterbody at all exit pressure ratios tested. Afterbody forces at the highest exit pressure ratio tested, $p_{e} / p_{\infty}=6.27$, were found to augment gross thrust by $5 \%$, and accounted for $95 \%$ of total lift. The aerodynamic center was found to move forward 0.5 body lengths from power-off to the maximum exit pressure ratio. Predicting such quantities accurately will be critical to designing a vehicle with minimal trim drag. In light of the current level of research activity in this field, these results portend the significant contribution CFD is now prepared to make to NASP and related hypersonics research.

Hypersonic results for the generic hypersonic vehicle proved fascinating from a fluid dynamics standpoint. The flow conditions prescribed here $\left(M_{\infty}=6, p_{e} / p_{\infty}=100, M_{e}=2.04\right)$, albeit arbitrarily chosen, are not unlike those spoken of for a hypersonic passenger transport. It was found that the exhaust plume shock induces crossflow separation, forming a lambda shock at the body. Vortical flows were found above the crossflow separation lines. Within the footprint region, very complicated flow patterns arise from the expanding flow and tapering body. Thrust vectoring was shown to weaken the plume/afterbody interaction. Because of the magnitude of the thrust, thrust vectoring could be critical to vehicle balance. 
Finally, the code was shown to be computationally efficient, both for generating solutions from freestream initial conditions, and reconverging perturbed solutions (for example, by changing the nozzle exit pressure ratio). A grid resolution study was performed for the nozzie/afterbody model, the results of which indicated that the grid dimensions chosen for these calculations were sufficient to ascertain integrated quantities, such as afterbody forces and moments, as well as resolve flow field details, such as crossflow separation patterns.

In conclusion, an important first step has been taken in solving the scramjet propulsion problem. Inevitably, an introductory investigation such as this raises more questions than it answers, though. Experience gained during the course of this investigation indicates several avenues along which further fruitful research may be pursued, and these are discussed below.

\subsection{Recommendations for Future Work}

One aspect of interest that is already under investigation concerns the effect of the inlet swallowing of some of the external flow on the afterbody flow field (private communication from J. Flores, NASA Ames Research Center, Moffett Field, California). In all the cases presented here, the inlet was not modeled. This effectively displaces more mass around the body, and one would expect this to result in a higher ambient pressure at the nozzle exit. Modeling the inlet with appropriate mass flow will effect a lower pressure environment at the nozzle exit, causing the plume to expand more rapidly into the external flow. The magnitude of this influence on afterbody forces needs to be determined.

Whenever discussions of flow separation and skin friction patterns arise, turbulence modeling becomes a central, controversial topic. Kaynak [48] implemented a relaxation modification to the BaldwinLomax model, the same turbulence model included in the UWIN code, that resulted in much better comparisons between predicted and experimental separation patterns. However, the method addressed only transonic shock-induced separation. It may be totally inappropriate for hypersonic flow, and regardless, an all-purpose hypersonic turbulence model (ideally including a transition model) would be of far greater value.

Stepwise advancements toward progressively more realistic flight conditions are certain to be forthcoming. Enhancements to look for include modeling equilibrium air chemistry for the external flow and equilibrium hydrogen-air chemistry for the plume flow. Allowing the streams to mix will also require a turbulent shear layer model and a model for turbulent diffusion. Solution-adaptive grids will doubtlessly be a necessity for such calculations. Finally, nonequilibrium chemistry in the plume will be necessary to predict very high speed, high altitude flight conditions.

Clearly, CFD research in hypersonic propulsion is still in its infancy. Analysis tools now being developed offer fertile ground for technical innovation. And for the first time since the inception of the science, computational fluid dynamics stands to play the lead role in the preliminary design of an entire vehicle. 


\title{
APPENDIX
}

\section{SIMPLIFYING ASSUMPTIONS FOR THE}

\author{
SPECIES CONTINUITY EQUATION
}

The full species continuity equation for a binary mixture was presented in equation (2.9). This equation was then simplified by neglecting the terms related to diffusion and production. The following pages present an order of magnitude analysis of the species continuity equation with application to the present problem. This analysis will show that, for the nozzle/afterbody problem addressed here, the diffusion terms may be neglected with negligible error, thereby permitting solution of a considerably simpler equation.

To begin, consider the full species continuity equation for a binary mixture, given in equation (2.9):

$$
\begin{aligned}
\frac{\partial\left(\rho c_{i}\right)}{\partial t}+\frac{\partial(\overbrace{\rho c_{i} u}^{\text {convection }}+\overbrace{j_{i_{x}}}^{\text {diffusion }})}{\partial x}+\frac{\partial\left(\rho c_{i} v+j_{i_{y}}\right)}{\partial y}+\frac{\partial\left(\rho c_{i} w+j_{i_{s}}\right)}{\partial z} & =\underbrace{K_{i}}_{\text {net production }}
\end{aligned}
$$

To arrive at the simplified equation, it must be shown that: 1) there is neither production nor destruction of species, and 2) the convection terms are much larger than the diffusion terms. The first condition is easily satisfied by noting that $\mathrm{SF}_{6}$ is inert in air, so no chemical reactions take place. Therefore, species can neither be produced nor destroyed and $K_{i} \equiv 0$.

To satisfy the second condition, inspect the diffusive mass flux vector, given in equation (2.10) as

$$
\mathbf{j}_{i}=\frac{n^{2}}{\rho} \sum_{j=1}^{\nu} m_{i} m_{j} D_{i j} \mathbf{d}_{j}-D_{i}^{T} \frac{\partial \ln (\mathrm{T})}{\partial \mathbf{r}} \quad i=1,2,3, \cdots \nu
$$

where $\mathbf{d}_{j}$, the macroscopic gradient vector, is given by

$$
\mathbf{d}_{j}=\underbrace{\frac{\partial\left(n_{j} / n\right)}{\partial \mathbf{r}}}_{\text {concentration }}+\underbrace{\left(\frac{n_{j}}{n}-\frac{n_{j} m_{j}}{\rho}\right) \frac{\partial \ln (\mathrm{p})}{\partial \mathbf{r}}}_{\text {pressure }}-\underbrace{\left(\frac{n_{j} m_{j}}{p \rho}\right)\left[\frac{\rho}{m_{j}} \mathbf{X}_{j}-\sum_{k=1}^{\nu} n_{k} \mathbf{X}_{k}\right]}_{\text {force }}
$$

The type of diffusion associated with each term is indicated beneath that term. Of these, concentration diffusion is the only one that may be important in equation (A.1). The second term, pressure diffusion, arises because heavier molecules move more slowly than light ones at the same pressure. In the presence 
of a pressure gradient, the net diffusion rate of light molecules toward the low pressure is greater than that of heavy molecules. Howe and Sheaffer [44] showed that this type of diffusion is important only in regions where a pressure gradient coincides with a nonuniform mixture $(0<c<1)$. Referring to figure 7 , it is seen that for an underexpanded jet flow field, such conditions exist only very close to the nozzle lip, where the exhaust gas first expands to ambient pressure. Elsewhere, the mixing layer occurs at the slip surface between the plume flow and the external How. Since the slip surface is by necessity one of constant pressure, pressure diffusion is negligible away from the nozzle lip. Force diffusion arises from body forces acting nonuniformly on the fluid. In keeping with the assumptions made for the Navier-Stokes equations, body forces are neglected and hence the third term in equation (A.3) vanishes.

Return now to equation (A.2), inserting the only surviving term from equation (A.3):

$$
\mathbf{j}_{i}=\underbrace{\frac{n^{2}}{\rho} \sum_{j=1}^{2} m_{i} m_{j} D_{i j} \frac{\partial\left(n_{j} / n\right)}{\partial \mathbf{r}}}_{\text {concentration }}-\underbrace{D_{i}^{T} \frac{\partial \ln (\mathrm{T})}{\partial \mathbf{r}}}_{\text {thermal }}
$$

Note that $\nu=2$ for the binary mixture assumption applied here. The thermal diffusion term arises from a similar mechanism to pressure diffusion: heavy molecules move more slowly than light molecules at the same temperature. Hence, in the presence of a temperature gradient, light molecules will migrate more rapidly to the lower temperature, effecting thermal diffusion. The nozzle/afterbody experiments employed cold jets, wherein the jet stagnation temperature was nearly equal to the freestream stagnation temperature. Therefore, temperature gradients in the flow field are small, and thermal diffusion is negligible.

Now expand the summation of equation (A.4) for the first species of the binary mixture:

$$
\begin{aligned}
\mathbf{j}_{1} & =\frac{n^{2} m_{1} D_{12}}{\rho}\left[m_{2} \frac{\partial\left(n_{2} / n\right)}{\partial \mathbf{r}}\right] \\
& =-\frac{n^{2} m_{1} m_{2} D_{12}}{\rho} \frac{\partial\left(n_{1} / n\right)}{\partial \mathbf{r}}
\end{aligned}
$$

where $\partial\left(n_{1} / n\right) / \partial \mathbf{r}=-\partial\left(n_{2} / n\right) / \partial \mathbf{r}$, and $D_{11}=0$. Using $n_{1}=\rho_{1} / m_{1}, \quad n=\rho / m$, and $c_{1}=\rho_{1} / \rho$, equation (A.5) simplifies to

Now let $n m_{2} \approx \rho$, giving

$$
\mathbf{j}_{1}=-n m_{2} D_{12} \frac{\partial c_{1}}{\mathbf{r}}
$$

$$
\mathbf{j}_{1} \approx-\rho D_{12} \frac{\partial c_{1}}{\partial \mathbf{r}}
$$

An approximate expression for the binary diffusion coefficient, $D_{12}$, is given in reference [42] as

$$
D_{12} \approx 2.628 \cdot 10^{-3} \frac{\sqrt{T^{3} / m}}{p \sigma^{2}}
$$

where

$$
\begin{aligned}
D & =\text { binary diffusion coefficient }, \mathrm{cm}^{2} / \mathrm{sec} \\
T & =\text { temperature in Kelvin } \\
m & =\text { molecular weight } \\
p & =\text { pressure in atmospheres } \\
\sigma & =\text { molecular diameter in } \AA
\end{aligned}
$$


This expression is valid only for self-diffusion, so to account for different species, an average mass is used: $m \approx m_{1} m_{2} / \frac{1}{2}\left(m_{1}+m_{2}\right)$. To obtain an order of magnitude estimate for the binary diffusion coefficient, the following values are estimated for the nozzle/afterbody experiment:

$$
\begin{aligned}
T & \approx T_{\infty}=100 \mathrm{~K} \\
m & \approx 46.18 \mathrm{~g} / \mathrm{gmole} \\
p & \approx p_{\infty}=.0158 \mathrm{~atm} \\
\sigma & \approx 10 \AA
\end{aligned}
$$

Thus, $D_{12} \approx 2.5 \mathrm{~cm}^{2} / \mathrm{sec}=O(1)$.

The concentration gradient is approximated as

$$
\frac{\partial c}{\partial \mathbf{r}} \approx \frac{c_{1}}{\delta}
$$

where $\delta$ is the shear layer thickness. Assuming $c_{1}$ changes from between 0 to 1 over the thickness of the shear layer, this term is of order $\delta^{-1}$. Shear layers are jet-like flows, for which the thickness scales with the distance (lecture notes from B. J. Cantwell, Stanford University, Stanford, California). Suppose $\delta \sim x / d_{e}$, where $d_{e}$ is the nozzle exit diameter. Away from the nozzle exit, then, the shear layer thickness will be on the order of 1 to $10 \mathrm{~cm}$ within the solution domain, and hence the concentration gradient will be, at most, $1 \mathrm{~cm}^{-1}$. Combining this with the approximate binary diffusion coefficient, the diffusive mass flux vector is

$$
\mathbf{j}_{1}=D_{12} \frac{\partial c_{1}}{\partial \mathbf{r}}=O(1) O(1)=O(1)
$$

Referring back to equation (A.1), $j_{1}$ appears in the same differential with uc. We already have $c=O(1)$, and if $u=O\left(u_{\infty}\right)=100,000 \mathrm{~cm} / \mathrm{sec}$, then the convective term is $O(100,000)$. Clearly diffusion is negligible where these assumptions hold.

Further analysis is necessary to determine the role diffusion in the nozzle exit region plays on the afterbody forces futher downstream. Another important source of diffusion, that resulting from turbulent convection, has been ignored altogether. Finally, in the boundary layer, where the flow velocity vanishes at the wall, diffusion also takes on greater importance. But because the pressure is nearly constant in the boundary layer, the surface forces are determined by the flow outside the boundary layer, where the foregoing assumptions are valid. Therefore, the diffusion terms are safely omitted for the flow fields presented here. 


\section{REFERENCES}

1. Rae, W. J.: New Challenges in Hypersonic Flight. Engineering Progress, vol. 6, no. 2, Winter-Spring 1987.

2. Bylinsky, G.: The 10,000-MPH Airliner. Fortune, December 8, 1986.

3. DeMeis, R.: An Orient Express to Capture the Market. Aerospace America, September 1987.

4. Anderson, G. Y.; Bencze, D. P.; and Sanders, R. W.: Ground Tests Confirm the Promise of Hypersonic Propulsion. Aerospace America, September 1987.

5. DeMeis, R.: Multimodes to Mach 5. Aerospace America, September 1987.

6. Johnston, P. J.; Whitehead, A. H., Jr.; and Chapman, G. T.: Fitting Aerodynamics and Propulsion into the Puzzle. Aerospace America, September 1987.

7. Mace, J.; and Hankey, W.: Review of Inlet-Airframe Integration Using Navier-Stokes Computational Fluid Dynamics. ALAA paper 84-0119, January 1984.

8. Tinoco, E.; and Chen, A.: Transonic CFD Applications to Engine/Airframe Integration. AIAA paper 84-0381, January 1984.

9. Flores, J.; Chaderjian, N.; and Sorenson, R.: Simulation of Transonic Viscous Flow over a Fighter-Like Configuration Including Inlet. AIAA paper 87-1199, July 1987.

10. Wolf, D.; Dash, S.; and Pergament, H.: A Shock-Capturing Model for Two-Phase, ChemicallyReacting Flow in Rocket Nozzles. AIAA paper 85-0306, January 1985.

11. Venkatapathy, E.; Lombard, C. K.; Bardina, J.; and Luh, R. C.-C.: Accurate Numerical Simulation of Supersonic Jet Exhaust Flow with CSCM on Adaptive Overlapping Grids. AIAA paper 87-0465, January 1987.

12. Petrie, H.; and Walker, B.: Comparison of Experiment and Computation for a Missile Base Region Flowfield with a Centered Propulsive Jet. AIAA paper 85-1618, July 1985.

13. Hoffman, J. J.; Birch, S. F.; Hopcroft, R. G.; and Holcomb, J. E.: Navier-Stokes Calculations of Rocket Base Flows. AIAA paper 87-0466, January 1987.

14. Cubbage, J. M., Jr.; and Kirkham, F. S.: Investigation of Engine-Exhaust-Airframe Interference on a Cruise Vehicle at Mach 6. NASA TN D-6060, 1971.

15. Small, W.; Weidner, J.; and Johnston, P.: Scramjet Nozzle Design and Analysis as Applied to a Highly Integrated Hypersonic Research Airplane. NASA TN D-8334, 1976. 
16. Penland, J. A.; Edwards, C. L. W.; Witcofski, R. D.; and Marcum, D. C., Jr.: Comparative Aerodynamic Study of Two Hypersonic Cruise Aircraft Configurations Derived from Trade-Off Studies. NASA TM X-1436, 1967.

17. Kirkham, F. S.; Cubbage, J. M., Jr.; Vahl, W. A.; and Small, W. J.: Studies of Airframe-PropulsionSystem Integration for Mach 6 Cruise Vehicles. NASA TN D-4128, 1967.

18. Henry, J.; and Anderson, G.: Design Considerations for the Airframe-Integrated Scramjet. NASA TM $\mathrm{X}-2895,1973$.

19. Edwards, C.: A Forebody Design Technique for Highly Integrated Bottom-Mounted Scramjets with Application to a Hypersonic Research Airplane. NASA TM X-71971, 1974.

20. Lockman, W. K.; Cleary, J. W.; and Lawrence, S. L.: Flow Visualization and Pressure Distribution for an All-Body Hypersonic Aircraft. Paper 53, Fourth National Aero-Space Plane Technology Symposium, February 1988.

21. Chapman, D.: Computational Aerodynamics Development and Outlook. AIAA Journal, vol. 17, 1979, pp. 1293-1313.

22. MacCormack, R.: The Effect of Viscosity in Hypervelocity Impact Cratering. AIAA paper 69-354, April 30-May 2, 1969.

23. Beam, R.; and Warming, R.: An Implicit Finite-Difference Algorithm for Hyperbolic Systems in Conservation-Law Form. Journal of Computational Physics, vol. 22, Sept. 1976, pp. 87-110.

24. Pulliam, T.; and Chaussee, D.: A Diagonal Form of an Implicit Approximate-Factorization Algorithm. Journal of Computational Physics, vol. 39, no. 2, 1981, pp. 347-363.

25. Rizk, Y.; and Ben-shmuel, S.: Computation of the Viscous Flow Around the Shuttle Orbiter at Low Supersonic Speeds. AIAA paper 85-0168, January 1985.

26. Steger, J. L.; and Warming, R. F.: Flux-Vector Splitting of the Inviscid Gas Dynamic Equations with Applications to Finite Difference Methods. Journal of Comp. Physics, vol. 40, 1981, pp. 263-293.

27. Roe, P.: Approximate Riemann Solvers, Parameter Vectors, and Difference Schemes. Journal of Comutational Physics, vol. 43, 1983, pp. 357-372.

28. Yee, H.: On Symmetric and Upwind TVD Schemes. NASA TM 86842, 1985.

29. Roe, P.: Generalized Formulation of TVD Lax-Wendroff Schemes. ICASE Report no. 84-53, 1984.

30. Rai, M.; and Chakravarthy, S.: An Implicit Form for the Osher Upwind Scheme. AIAA Journal, vol. 24 , no. 5,1986 , pp. $735-743$.

31. Lawrence, S.; Tannehill, J.; and Chaussee, D.: Application of an Upwind Algorithm to the ThreeDimensional Parabolized Navier-Stokes Equations. AIAA paper 87-1112, July 1987. 
32. Rai, M.: An Upwind, Patched-Grid Approach to Solving the Unsteady Thin-Layer Navier-Stokes Equations in Three Dimensions. NASA Technical Memorandum in preparation.

33. Edwards, T.; Chaussee, D.; Lawrence, S.; and Rizk, Y.: Comparisons of Four CFD Codes as Applied to a Hypersonic All-Body Vehicle. AIAA paper 87-2642, August 1987.

34. Newberry, C. F.; Dresser, H. S.; Byerly, J. W.; and Riba, W. T.: The Evaluation of Forebody Compression at Hypersonic Mach Numbers. AIAA paper 88-0479, January 1988.

35. Barber, T. J.; and Cox, G. B., Jr.: Hypersonic Vehicle Propulsion: A CFD Application Case Study. AIAA paper 88-0475, January 1988.

36. Bergman, B. K.; and Treiber, D. A.: The Application of Euler and Navier-Stokes Methodology to 2-D and 3-D Nozzle-Afterbody Flowfields. AIAA paper 88-0274, January 1988.

37. Gielda, T. P.; Hunter, L. G.; and Chawner, J. R.: Efficient Parabolized Navier-Stokes Solutions of Three-Dimensional, Chemically Reacting Scramjet Flowfields. AIAA paper 88-0096, January 1988.

38. Chitsomboon, T.; Rogers, R. C.; Northam, G. B.; Jarrett, O., Jr.; and Antcliff, R. R.: Supersonic Combusting Flows: A Comparison of Numerical and Experimental Results. AIAA paper 88-0095, January 1988.

39. Balakrishnan, A.: Computation of a Viscous Real Gas Flowfield for the Space Shuttle Orbiter. AIAA paper 84-1748, June 1984.

40. Molvik, G. A.: A Parabolized Navier-Stokes Code with Real Gas Effects. Paper presented at AFWAL PNS Code User's Workshop, September 1986, Dayton, Ohio.

41. Balakrishnan, A.: Application of a Flux-Split Algorithm to Chemically Relaxing, Hypervelocity BluntBody Flows. AIAA paper 87-1578, June 1987.

42. Hirschfelder, J.; Curtiss, C.; and Bird, R.: Molecular Theory of Gases and Liquids. John Wiley \& Sons, Inc., N.Y., 1964.

43. Ahtye, W.: A Critical Evaluation of Methods for Calculating Transport Coefficients of Partially and Fully Ionized Gases. NASA TN D-2611, 1965.

44. Howe, J.; and Sheaffer, Y.: Role of Charge Separation and Pressure Diffusion in the Gascap of Entry Objects. AIAA Journal, vol. 7, no. 10, October 1969, pp. 1971-1977.

45. Vincenti, W.; and Kruger, C.: Introduction to Physical Gas Dynamics. Robert E. Krieger Publishing Company, Malabar, Florida, 1982.

46. Yee, H. C.: Numerical Experiments with a Symmetric High-Resolution Shock-Capturing Scheme. NASA TM 88325, 1986.

47. Baldwin, B. S.; and Lomax, H.: Thin-Layer Approximation and Algebraic Model for Separated Turbulent Flows. AIAA paper 78-257, January 1978. 
48. Kaynak, U.; Holst, T. L.; and Cantwell, B. J.: Computation of Transonic Separated Wing Flows Using an Euler/Navier-Stokes Zonal Approach. NASA TM 88311, 1986.

49. Benek, J. A.; Steger, J. L.; and Dougherty, F. C.: A Flexible Grid Embedding Technique with Application to the Euler Equations. AIAA paper 83-1944, July 1983.

50. Rai, M.: An Implicit, Conservative, Zonal-Boundary Scheme for Euler Equation Calculations. Computers \& Fluids, vol. 14, no. 3, 1986, pp. 295-319.

- 51. Buning, G. P.; and Steger, J. L.: Graphics and Flow Visualization in Computational Fluid Dynamics. AIAA paper 85-1507, July 1985.

52. Ames Research Staff: Equations, Tables, and Charts for Compressible Flow. Report 1135, 1953.

53. Steger, J. L.; and Chaussee, D. S.: Generation of Body-Fitted Coordinates Using Hyperbolic Partial Differential Equations. SIAM J. Sci. Stat. Comput., vol. 1, no. 4, 1980, pp. 431-437.

54. Ying, S.; Steger, J. L.; Schiff, L. B.; and Baganoff, D.: Numerical Simulation of Unsteady, Viscous, High Angle of Attack Flows Using a Partially Flux Split Algorithm. AIAA paper 86-2179, 1986.

55. Reznick, S.: Transonic Navier-Stokes Computations of Strake-Generated Vortex Interactions for a Fighter-Like Configuration. NASA TM 100009, 1988. 


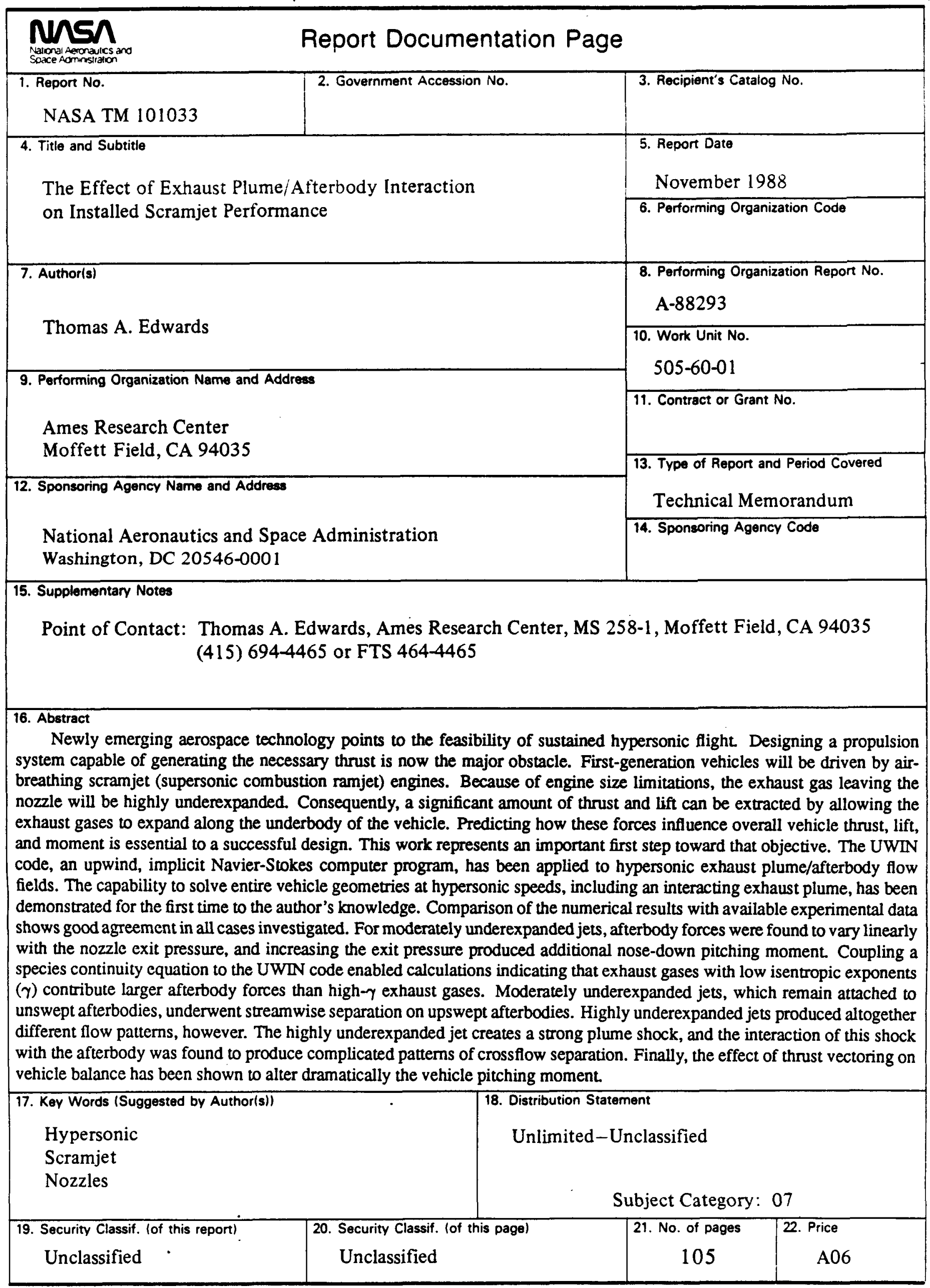

